\title{
SHARP TRACE THEOREMS FOR NULL HYPERSURFACES ON EINSTEIN METRICS WITH FINITE CURVATURE FLUX
}

\author{
SERGIU KLAINERMAN AND IGOR RODNIANSKI
}

\begin{abstract}
The main objective of the paper is to prove a geometric version of sharp trace and product estimates on null hypersurfaces with finite curvature flux. These estimates play a crucial role to control the geometry of such null hypersurfaces. The paper is based on an invariant version of the classical Littlewood -Paley theory, in a noncommutative setting, defined via heat flow on surfaces.
\end{abstract}

\section{INTRODUCTION}

To motivate the problems studied in this paper we start with the simplest example of a sharp trace theorem. This applies to smooth functions $f=f\left(t, x^{1}, x^{2}\right)$ on $I \times \mathbb{R}^{2} \subset \mathbb{R}^{3}$ with $I$ an interval in $\mathbb{R}$, for simplicity $I=[0,1]$. We denote by \|\|$_{L^{2}}$ the standard $L^{2}$ norm on $I \times \mathbb{R}^{2}$ and by \|\|$_{H^{k}}, k$ positive integer, the usual norm of the Sobolev space $H^{k}\left(I \times \mathbb{R}^{2}\right)$. Thus,

$$
\begin{aligned}
\|f\|_{L^{2}}=\|f\|_{L^{2}\left(I \times \mathbb{R}^{2}\right)} & =\left(\int_{0}^{1} \int_{\mathbb{R}^{2}}|f(t, x)|^{2} d t d x\right)^{\frac{1}{2}} \\
\|f\|_{H^{k}}=\|f\|_{H^{k}\left(I \times \mathbb{R}^{2}\right)} & =\left(\sum_{i+j \leq k} \int_{0}^{1} \int_{\mathbb{R}^{2}}\left|\partial_{t}^{i} \nabla^{j} f(t, x)\right|^{2} d t d x\right)^{\frac{1}{2}}
\end{aligned}
$$

with $\nabla^{j}$ the partial derivatives of order $j$ with respect to the $x$ variables. We shall also use the mixed norm notation,

$$
\begin{aligned}
\|f\|_{L_{t}^{q} L_{x}^{p}} & =\left(\int_{0}^{1}\|f(t, \cdot)\|_{L^{p}\left(\mathbb{R}^{2}\right)}^{q} d t\right)^{\frac{1}{q}} \\
\|f\|_{L_{x}^{p} L_{t}^{q}} & =\left(\int_{\mathbb{R}^{2}}\|f(\cdot, x)\|_{L_{t}^{q}(I)}^{p} d x\right)^{\frac{1}{p}}
\end{aligned}
$$

with the obvious modifications when either $p=\infty$ or $q=\infty$.

Proposition 1.1. The following inequality holds for an arbitrary, smooth, scalar function $f=f\left(t, x^{1}, x^{2}\right)$ in $\mathbb{R}^{3}$ :

$$
\left\|\partial_{t} f\right\|_{L_{x}^{\infty} L_{t}^{2}\left(I \times \mathbb{R}^{2}\right)} \lesssim\|f\|_{H^{2}\left(I \times \mathbb{R}^{2}\right)}
$$

1991 Mathematics Subject Classification. 35J10

The first author is partially supported by NSF grant DMS-0070696. The second author is a Clay Prize Fellow and is partially supported by NSF grant DMS-01007791. 
The inequality can be easily derived with the help of the $W^{2,1}\left(\mathbb{R}^{2}\right) \subset L^{\infty}$ imbedding and a standard integration by parts. Observe that the estimate is false if one replaces $\partial_{t}$ with the other partial derivatives $\partial_{x^{1}}, \partial_{x^{2}}$.

Using the a standard Littlewood-Paley theory it is not too difficult to prove a stronger version of (1) in Besov spaces ${ }^{1}$.

$$
\left\|\int_{I}\left|\partial_{t} f\right|^{2} d t\right\|_{B_{2,1}^{1}\left(\mathbb{R}^{2}\right)} \lesssim\|f\|_{H^{2}\left(I \times \mathbb{R}^{2}\right)}^{2}
$$

Here, for a function $g=g\left(x^{1}, x^{2}\right)$,

$$
\|g\|_{B_{2,1}^{\theta}\left(\mathbb{R}^{2}\right)}=\sum_{k \geq 0} 2^{k \theta}\left\|P_{k} g\right\|_{L^{2}\left(\mathbb{R}^{2}\right)}+\left\|P_{<0} g\right\|_{L^{2}\left(\mathbb{R}^{2}\right)}
$$

denotes the standard, inhomogeneous, Besov norm in $\mathbb{R}^{2}$ with $\theta \geq 0$ and $P_{k}$ the usual Littlewood Paley (LP) projections, see for example [Stein1], [Stein2] and[B] for applications to paradifferential calculus. Also, $P_{<0}=\sum_{k<0} P_{k}$. Observe that,

$$
\|g\|_{B_{2,1}^{1}} \lesssim\|\nabla g\|_{B_{2,1}^{0}}+\|g\|_{L^{2}}
$$

and therefore (2) follows easily from its following bilinear version:

Proposition 1.2 ( Sharp bilinear trace). The following inequality holds for an arbitrary, smooth, scalar function $g, h$ on $I \times \mathbb{R}^{2}$ :

$$
\left\|\int_{I} \partial_{t} g \cdot h\right\|_{B_{2,1}^{0}\left(\mathbb{R}^{2}\right)} \lesssim\|g\|_{H^{1}\left(I \times \mathbb{R}^{2}\right)} \cdot\|h\|_{H^{1}\left(I \times \mathbb{R}^{2}\right)}
$$

In addition to the bilinear sharp trace estimate (3) we also signal the following related estimate,

Proposition 1.3 ( Sharp integrated product). The following inequality holds for an arbitrary, smooth, scalar function $g, h$ on $I \times \mathbb{R}^{2}$ :

$$
\left\|\int_{I} g \cdot h\right\|_{B_{2,1}^{0}\left(\mathbb{R}^{2}\right)} \lesssim\left(\|g\|_{H^{1}\left(I \times \mathbb{R}^{2}\right)}+\|g\|_{L_{x}^{\infty} L_{t}^{2}}\right) \cdot\|h\|_{L_{t}^{2} B_{x}^{0}}
$$

where,

$$
\|h\|_{L_{t}^{q} B_{x}^{0}}=\left(\int_{0}^{1}\|h(t, \cdot)\|_{B_{2,1}^{0}\left(\mathbb{R}^{2}\right)}^{q} d t\right)^{1 / q} .
$$

Also,

$$
\left\|g \cdot \int_{0}^{t} h\right\|_{L_{t}^{2} B_{x}^{0}} \lesssim\left(\|g\|_{H^{1}\left(I \times \mathbb{R}^{2}\right)}+\|g\|_{L_{x}^{\infty} L_{t}^{2}}\right) \cdot\|h\|_{L_{t}^{1} B_{x}^{0}}
$$

Remark 1.4. Proposition 1.3 is intimately related with the following well known estimate for functions in $\mathbb{R}^{2}$ :

$$
\|f \cdot g\|_{B_{2,1}^{0}\left(\mathbb{R}^{2}\right)} \lesssim\left(\|g\|_{H^{1}\left(\mathbb{R}^{2}\right)}+\|g\|_{L^{\infty}\left(\mathbb{R}^{2}\right)}\right) \cdot\|h\|_{B_{2,1}^{0}\left(\mathbb{R}^{2}\right)}
$$

\footnotetext{
${ }^{1}$ This is not just a minor technical improvement. It turns out that this type of Besov space improvement of the sharp trace estimate plays a fundamental role in [Kl-Rodn1].
} 
Propositions 1.1 and 1.3 can be easily reformulated in terms of functions defined on null hypersurfaces in Minkowski space $\mathbb{R}^{3+1}$. For simplicity consider the standard null hypersurface defined by the equation $u=-1$ where $u$ is the optical function $u=t-r, r=\sqrt{\sum_{i=1}^{3}\left(x^{i}\right)^{2}}$. Let $\mathcal{H}$ denote the portion of this null hypersurface contained between $t=0$ and $t=1$. An arbitrary null geodesic $\Gamma=\Gamma_{\omega}$ along $\mathcal{H}$ can be parametrized by $(t, t \omega)$ where $\omega$ is a unit vector in $\mathbb{R}^{3}$. Given a scalar function $f$ on $\mathcal{H}$ we denote by $\int_{\Gamma_{\omega}} f=\int_{0}^{1} f(t, t \omega) d t$. We denote by $\nabla_{L} f$ its derivative along the null geodesic, i.e. $\nabla_{L} f=\frac{d}{d t} f(t, t \omega)$. We also denote by $\nabla f$ the angular derivatives of $f$ and by $\bar{\nabla} f$ all tangential derivatives of $f$ along $\mathcal{H}$, i.e. $\bar{\nabla} f=\left(\nabla_{L} f, \nabla f\right)$. To adapt proposition 1.2 to the case of the null hypersurface $\mathcal{H}$ we need to define a Besov space $B_{2,1}^{0}\left(\mathbb{S}^{2}\right)$ analogous to $B_{2,1}^{0}\left(\mathbb{R}^{2}\right)$ with $\mathbb{S}^{2}$ the standard unit sphere in $\mathbb{R}^{3}$. LP projections can, of course, be easily defined locally, in coordinate charts, and extended to all of $\mathbb{S}^{2}$ by a partition of unity. Besov spaces on $\mathbb{S}^{2}$ can then be formally introduced as before. A more intrinsic way to define such spaces would be based on spherical harmonic decomposition. Yet another way to achieve the same result is to introduce a definition of LP projections based on a heat flow for the corresponding Laplace-Beltrami operator on the leaves of the geodesic sphere foliation of $\mathcal{H}$ given by the level surfaces of the standard time function $t$, see (12). This is in fact the approach we develop here to deal with null hypersurfaces in non flat spacetimes.

The propositions below are straightforward adaptations of propositions 1.2, 1.3 to the case of the null hypersurface $\mathcal{H}$. The norms used in the proposition are,

$$
\begin{aligned}
\|f\|_{L^{2}(\mathcal{H})} & =\left(\int_{0}^{t} \int|g(t, t \omega)|^{2} d t d \omega\right)^{\frac{1}{2}} \\
\mathcal{N}_{1}(g) & =\left\|\nabla_{L} f\right\|_{L^{2}(\mathcal{H})}+\|\nabla f\|_{L^{2}(\mathcal{H})}+\|f\|_{L^{2}(\mathcal{H})} \\
\|f\|_{L_{x}^{\infty} L_{t}^{2}} & =\sup _{\omega \in \mathbb{S}^{2}}\left(\int_{\Gamma_{\omega}}|f|^{2}\right)^{\frac{1}{2}} \\
\|f\|_{\mathcal{P}^{0}} & =\left(\int\|f(t, t \cdot)\|_{B_{2,1}^{0}\left(\mathbb{S}^{2}\right)}^{2} d t\right)^{\frac{1}{2}}
\end{aligned}
$$

Proposition 1.5. Let $g, h$ be arbitrary smooth functions on $\mathcal{H}$. then the following estimates hold true, uniformly in $\omega \in \mathbb{S}^{2}$,

$$
\begin{gathered}
\left\|\int_{\Gamma_{\omega}}\left(\nabla_{L} g \cdot h\right)\right\|_{B_{2,1}^{0}\left(\mathbb{S}^{2}\right)} \lesssim \mathcal{N}_{1}(g) \cdot \mathcal{N}_{1}(h) \\
\left\|\int_{\Gamma_{\omega}} g \cdot h\right\|_{B_{2,1}^{0}\left(\mathbb{S}^{2}\right)} \lesssim\left(\mathcal{N}_{1}(g)+\|g\|_{L_{x}^{\infty} L_{t}^{2}}\right) \cdot\|h\|_{\mathcal{P}^{0}} \\
\left\|g \cdot \int_{\Gamma_{\omega}} h\right\|_{\mathcal{P}^{0}} \lesssim\left(\mathcal{N}_{1}(g)+\|g\|_{L_{x}^{\infty} L_{t}^{2}}\right) \cdot\|h\|_{\mathcal{P}^{0}}
\end{gathered}
$$

As a corollary of (7) we have the standard sharp trace theorem,

$$
\sup _{\omega \in \mathbb{S}^{2}} \int_{\Gamma_{\omega}}\left|\nabla_{L} f\right|^{2} \lesssim \mathcal{N}_{2}(f)^{2}
$$

with,

$$
\mathcal{N}_{2}(f)=\left\|\nabla_{L}^{2} f\right\|_{L^{2}(\mathcal{H})}+\left\|\nabla \nabla_{L} f\right\|_{L^{2}(\mathcal{H})}+\left\|\nabla^{2} f\right\|_{L^{2}(\mathcal{H})}+\mathcal{N}_{1}(f)
$$


The goal of this paper is to adapt the estimates (7), (8) and (10) to null hypersurfaces in curved backgrounds, verifying the Einstein-Vacuum equations, and verifying the bounded curvature flux(BCF) condition of [Kl-Rodn1]. These results, which formed the content of the main lemma in [Kl-Rodn1], played a crucial role in that paper. We recall that the main result of [Kl-Rodn1] was to prove that the (BCF) condition suffices to control the local geometry of null hypersurfaces. The (BCF) condition comes naturally in connection with the bounded $L^{2}$ curvature conjecture and the result of [Kl-Rodn1] is a crucial ingredient in the resolution of that conjecture.

To illustrate the techniques needed in our work we give, in section 2 , a quick proof of propositions 1.2 and 1.3. They are based on the properties of the standard, euclidean, Littlewood -Paley(LP) projections $P_{k}$ which we recall below.

The LP-projections $\left(P_{k}\right)_{k \in \mathbb{Z}}$, acting on functions $g(x), x \in \mathbb{R}^{2}$, are defined as Fourier multipliers according to the formula,

$$
\left(P_{k} f\right)^{\wedge}(\xi)=\chi\left(2^{-k} \xi\right) f^{\wedge}(\xi)
$$

with $f^{\wedge}(\xi)$ denoting the Fourier transform of $f$ and $\chi(\xi)=\chi(|\xi|)$ a real smooth test function supported in $\frac{1}{2} \leq|\xi| \leq 2$. Moreover, for all $\xi \in \mathbb{R}^{2} \backslash 0, \quad \sum_{k} \chi\left(2^{-k} \xi\right)=1$. We denote $P_{J}=\sum_{k \in J} P_{k}$ for all intervals $J \subset \mathbb{Z}$.

The following properties are at the heart of the classical LP theory:

LP 1. Almost Orthogonality: The operators $P_{k}$ are selfadjoint and verify $P_{k_{1}} P_{k_{2}}=0$ for all pairs of integers such that $\left|k_{1}-k_{2}\right| \geq 2$. In particular,

$$
\|f\|_{L^{2}} \approx \sum_{k}\left\|P_{k} f\right\|_{L^{2}}
$$

LP 2. $\quad L^{p}$-boundedness: $\quad$ For any $1 \leq p \leq \infty$, and any interval $J \subset \mathbb{Z}$,

$$
\left\|P_{J} f\right\|_{L^{p}} \lesssim\|f\|_{L^{p}}
$$

LP 3. Finite band property: We can write any partial derivative $\nabla P_{k} f$ in the form $\nabla P_{k} f=2^{k} \tilde{P}_{k} f$ where $\tilde{P}_{k}$ are the LP-projections associated with a slightly different test function $\tilde{\chi}$ and verify the property LP2. Thus, in particular, for any $1 \leq p \leq \infty$

$$
\begin{aligned}
\left\|\nabla P_{k} f\right\|_{L^{p}} & \lesssim 2^{k}\|f\|_{L^{p}} \\
2^{k}\left\|P_{k} f\right\|_{L^{p}} & \lesssim\|\nabla f\|_{L^{p}}
\end{aligned}
$$

LP 4. Bernstein inequalities. For any $2 \leq p \leq \infty$ we have the Bernstein inequality and its dual,

$$
\left\|P_{k} f\right\|_{L^{\infty}} \lesssim 2^{k}\|f\|_{L^{2}}, \quad\left\|P_{k} f\right\|_{L^{2}} \lesssim 2^{k}\|f\|_{L^{1}}
$$


LP 5. Commutation properties Given functions $f(t, x)$, if we denote by $P_{k} f$ the action of the LP projections in $x$, we have, trivially

$$
\partial_{t} P_{k} f=P_{k} \partial_{t} f, \quad P_{k} \int_{0}^{1} f d t=\int_{0}^{1} P_{k} f d t
$$

The proof of proposition 1.2 is a typical illustration of the power of paradifferential calculus. Clearly one needs, somehow, to integrate by parts, but because of the symmetry of the estimate (3) with respect to $f, g$ one does not achieve anything by a direct integration by parts. The idea is to decompose both functions $f=\sum_{k^{\prime}} P_{k^{\prime}} f$ and $g=\sum_{k^{\prime \prime}} P_{k^{\prime \prime}} g$ in the bilinear expression $\int_{0}^{1} \nabla_{L} f \cdot g$ and integrate by parts only those integral terms $\int_{0}^{t}\left(\partial_{t} f_{k^{\prime}} \cdot g_{k^{\prime \prime}}\right)$ where $k^{\prime} \geq k^{\prime \prime}$. This illustrates a general philosophy; the paradifferential calculus gives us the flexibility to deal differently with various parts of nonlinear expressions and thus allows us to separate and focus on various difficulties of the problem at hand. It is truly a divide and conquer strategy.

In order to extend the results mentioned below to general null hypersurfaces $\mathcal{H}$ we need to replace the LP theory based on Fourier transform with a more intrinsic geometric definition. Given a Riemannian manifold $M$ we can define LP projections $P_{k}$ according to the formula,

$$
P_{k} F=\int_{0}^{\infty} m_{k}(\tau) U(\tau) F d \tau
$$

where $m_{k}(\tau)=2^{2 k} m\left(2^{2 k} \tau\right)$ and $m(\tau)$ is a Schwartz function with a finite number of vanishing moments. The operator $U(\tau) F$ denotes the unique solution of the heat flow on $S$ with initial data provided by $F$,

$$
\partial_{\tau} U(\tau) F-\Delta U(\tau) F=0, \quad U(0) F=F .
$$

where $\Delta$ denotes the standard Laplace-Beltrami operator for tensors, $\Delta=\gamma^{i j} \nabla_{i} \nabla_{j}$. We apply this definition to the 2-dimensional leaves of the geodesic foliation on our null hypersurface $\mathcal{H}$. Under some simple assumptions on the geometry of these leaves we prove, in [Kl-Rodn2], a sequence of properties of the LP projections similar to LP1, LP4. Some of our results are, of course, weaker. For example the pointwise version of the almost orthogonality property LP5 cannot possible be true. We can replace it however by a sufficiently robust $L^{p}$ analogue of it. We also find satisfactory analogues for LP2-LP3, though we have to be quite careful about what we can in fact prove with our very limited regularity assumptions. For example, we can prove a version of the Bernstein inequalities of LP4 for scalars $f$ but not for tensorfields. Of course, LP5 plays a fundamental role in the proof of propositions 1.2, 1.3. Such a property, however, does not hold for the nonflat backgrounds we deal with in our work. This lack of commutativity compounded by the weak regularity properties of the foliation, consistent with the (BCF) assumption, leads to considerable conceptual and technical difficulties.

Once we have set up a satisfactory geometric LP theory we can formulate and prove results, on non flat backgrounds, similar to those of proposition 1.5. We prefer to state these results only after a thorough discussion of the geometric framework and the properties of the intrinsic LP projections. 
We give complete proofs of both propositions 1.2, 1.3 in section 2 in order to prepare the reader for the methods used in the non flat situation.

In section 3 we discuss the main geometric notions concerning null hypersurfaces in a curved background. We also introduce our main assumptions BA1, BA2, WS, K1 and K2. All these assumptions are consistent with the bounded curvature flux (BCF) condition of [Kl-Rodn1]. The main results of this paper depend only on these assumptions. We also introduce our geometric LP projections and state their main properties, proved in [Kl-Rodn2]. Finally we define our main Besov spaces and recall some of the properties proved in [Kl-Rodn2].

In section 4 we state a sequence of theorems which extend the results of (1.5) to nonflat backgrounds. These results were stated in section 5 of [Kl-Rodn1], and played an essential role in the proof of the main result there.

The remaining sections of the paper contain the proofs of these results.

\section{Proof of PRopositions 1.2 AND 1.3}

We shall make use of the properties P1-P5 of the classical LP projections mentioned in the introduction.

2.1. Proof of proposition 1.2. By definition,

$$
\begin{aligned}
\left\|\int_{0}^{1} \partial_{t} g \cdot h d t\right\|_{B_{2,1}^{0}} & =\sum_{k \geq 0}\left\|P_{k} \int_{0}^{1} \partial_{t} g \cdot h d t\right\|_{L_{x}^{2}}+\left\|P_{<0} \int_{0}^{1} \partial_{t} g \cdot h d t\right\|_{L_{x}^{2}} \\
& \lesssim \sum_{k}\left\|P_{k} \int_{0}^{1} \partial_{t} g \cdot h d t\right\|_{L_{x}^{2}}
\end{aligned}
$$

We decompose, with respect to the $x$ variables, $f=\sum_{k} f_{k}, g=\sum_{k} g_{k}$ with $f_{k}=$ $P_{k} f, g_{k}=P_{k} g$, and write,

$$
\begin{array}{cc}
P_{k} \int_{0}^{1}\left(\partial_{t} g \cdot h\right)=A_{k}+B_{k}+C_{k}+D_{k} \\
A_{k}=P_{k} \int_{0}^{1}\left(\partial_{t} g\right)_{<k} \cdot h_{\geq k}, \quad B_{k}=P_{k} \int_{0}^{1}\left(\partial_{t} g\right)_{\geq k} \cdot h_{<k} \\
C_{k}=P_{k} \int_{0}^{1}\left(\partial_{t} g\right)_{<k} \cdot h_{<k}, \quad D_{k}=P_{k} \int_{0}^{1}\left(\partial_{t} g\right)_{\geq k} \cdot h_{\geq k}
\end{array}
$$

Observe that $P_{k}$ commute with the integral $\int_{0}^{1}$ and that $C_{k}$ is essentially ${ }^{2}$ zero. Thus we only have to estimate $A_{k}, B_{k}, D_{k}$.

\footnotetext{
${ }^{2}$ With the possible exception of a finite, $\lesssim 8$, number of terms which can be made part of either $A_{k}, B_{k}, D_{k}$. This corresponds to the classical trichotomy formula and is due to LP1.)
} 
1.) Estimates for $A_{k}=P_{k} \int_{0}^{t}\left(\partial_{t} g\right)_{<k} \cdot h_{\geq k}$ : We use first $\mathbf{L P 2}$ followed by the the direct Bernstein inequality LP4, and then LP3, LP2. We also use LP5 freely.

$$
\begin{aligned}
\left\|A_{k}\right\|_{L_{x}^{2}} & \lesssim \sum_{k^{\prime}<k \leq k^{\prime \prime}} \int_{0}^{1}\left\|\left(\partial_{t} g\right)_{k^{\prime}} \cdot h_{k^{\prime \prime}}\right\|_{L_{x}^{2}} d t \lesssim \sum_{k^{\prime}<k \leq k^{\prime \prime}} \int_{0}^{1}\left\|\left(\partial_{t} g\right)_{k^{\prime}}\right\|_{L_{x}^{\infty}} \cdot\left\|h_{k^{\prime \prime}}\right\|_{L_{x}^{2}} d t \\
& \lesssim \sum_{k^{\prime}<k \leq k^{\prime \prime}} 2^{k^{\prime}} \int_{0}^{1}\left\|\left(\partial_{t} g\right)_{k^{\prime}}\right\|_{L_{x}^{2}} \cdot\left\|h_{k^{\prime \prime}}\right\|_{L_{x}^{2}} d t \\
& \lesssim \sum_{k^{\prime}<k \leq k^{\prime \prime}} 2^{k^{\prime}-k^{\prime \prime}}\left\|\left(\partial_{t} g\right)_{k^{\prime}}\right\|_{L_{t}^{2} L_{x}^{2}} \cdot\left\|\nabla h_{k^{\prime \prime}}\right\|_{L_{t}^{2} L_{x}^{2}}
\end{aligned}
$$

Therefore, writing $2^{k^{\prime}-k^{\prime \prime}}=2^{\left(k^{\prime}-k\right) / 2+\left(k^{\prime}-k^{\prime \prime}\right) / 2} \lesssim 2^{\left(k^{\prime}-k\right) / 2+\left(k-k^{\prime \prime}\right) / 2}$, summing over $k$ and using LP1,

$$
\begin{aligned}
\sum_{k \geq 0}\left\|A_{k}\right\|_{L_{t}^{\infty} L_{x}^{2}} & \lesssim \sum_{k} \sum_{k^{\prime}<k \leq k^{\prime \prime}} 2^{\left(k^{\prime}-k^{\prime \prime}\right) / 2} 2^{\left(k-k^{\prime \prime}\right) / 2}\left\|\left(\partial_{t} g\right)_{k^{\prime}}\right\|_{L_{t}^{2} L_{x}^{2}}\left\|\nabla h_{k^{\prime \prime}}\right\|_{L_{t}^{2} L_{x}^{2}} \\
& \lesssim \sum_{k^{\prime}<k^{\prime \prime}} 2^{\left(k^{\prime}-k^{\prime \prime}\right) / 2}\left\|\left(\partial_{t} g\right)_{k^{\prime}}\right\|_{L_{t}^{2} L_{x}^{2}}\left\|\nabla h_{k^{\prime \prime}}\right\|_{L_{t}^{2} L_{x}^{2}} \\
& \lesssim\left\|\partial_{t} g\right\|_{L_{t}^{2} L_{x}^{2}} \cdot\|\nabla h\|_{L_{t}^{2} L_{x}^{2}} .
\end{aligned}
$$

2.) Estimates for $D_{k}=P_{k} \int_{0}^{t}\left(\partial_{t} F\right)_{\geq k} \cdot G_{\geq k}$ : We write, $D_{k}=D_{k}^{1}+D_{k}^{2}$ where,

$$
D_{k}^{1}=\sum_{k \leq k^{\prime} \leq k^{\prime \prime}} P_{k} \int_{0}^{1}\left(\partial_{t} g\right)_{k^{\prime}} \cdot h_{k^{\prime \prime}}, \quad D_{k}^{2}=\sum_{k \leq k^{\prime}<k^{\prime \prime}} P_{k} \int_{0}^{1}\left(\partial_{t} g\right)_{k^{\prime \prime}} \cdot h_{k^{\prime}}
$$

The term $D_{k}^{1}$ can be treated in a straightforward manner, without integration by parts. We start by using the dual Bernstein inequality LP4, followed by the finite band property LP3,

$$
\begin{aligned}
& \left\|D_{k}^{1}\right\|_{L_{x}^{2}} \lesssim 2^{k} \sum_{k \leq k^{\prime} \leq k^{\prime \prime}}\left\|\int_{0}^{1}\left(\partial_{t} g\right)_{k^{\prime}} \cdot h_{k^{\prime \prime}}\right\|_{L_{x}^{1}} \lesssim 2^{k} \sum_{k \leq k^{\prime} \leq k^{\prime \prime}}\left\|\partial_{t} g_{k^{\prime}}\right\|_{L_{t}^{2} L_{x}^{2}} \cdot\left\|h_{k^{\prime \prime}}\right\|_{L_{t}^{2} L_{x}^{2}} \\
& \lesssim 2^{k-k^{\prime \prime}}\left\|\partial_{t} g_{k^{\prime}}\right\|_{L_{t}^{2} L_{x}^{2}} \cdot\left\|\nabla h_{k^{\prime \prime}}\right\|_{L_{t}^{2} L_{x}^{2}}
\end{aligned}
$$

Thus, summing in $k$ and using the $L^{2}$ orthogonality property LP1

$$
\begin{aligned}
\sum_{k \geq 0}\left\|D_{k}^{1}\right\|_{L_{x}^{2}} & \lesssim \sum_{k} \sum_{k \leq k^{\prime}<k^{\prime \prime}} 2^{k-k^{\prime \prime}}\left\|\partial_{t} g_{k^{\prime}}\right\|_{L_{t}^{2} L_{x}^{2}} \cdot\left\|\nabla h_{k^{\prime \prime}}\right\|_{L_{t}^{2} L_{x}^{2}} \\
& \lesssim \sum_{k^{\prime}<k^{\prime \prime}} 2^{k^{\prime}-k^{\prime \prime}}\left\|\partial_{t} g_{k^{\prime}}\right\|_{L_{t}^{2} L_{x}^{2}} \cdot\left\|\nabla h_{k^{\prime \prime}}\right\|_{L_{t}^{2} L_{x}^{2}} \lesssim\left\|\partial_{t} g\right\|_{L_{t}^{2} L_{x}^{2}} \cdot\|\nabla h\|_{L_{t}^{2} L_{x}^{2}}
\end{aligned}
$$

To treat $D_{k}^{2}$ we need to transfer the $\partial_{t}$ derivative from the low frequency term $g_{k^{\prime}}$ to the high frequency term $h_{k^{\prime \prime}}$. After integration by parts we can treat the resulting integral exactly as $D_{k}^{1}$, the only terms we need to worry about are the boundary terms $\left\|I_{k}(1)-I_{k}(0)\right\|_{L_{x}^{2}} \lesssim \sup _{0 \leq t \leq 1}\left\|I_{k}(t, \cdot)\right\|_{L_{x}^{2}}=\left\|I_{k}\right\|_{L_{t}^{\infty} L_{x}^{2}}$, where

$$
I_{k}=\sum_{k \leq k^{\prime}<k^{\prime \prime}} P_{k}\left(g_{k^{\prime \prime}} \cdot h_{k^{\prime}}\right) \text {. }
$$

To treat them we need the following

Lemma 2.2. For any $k, k^{\prime}, k^{\prime \prime}$, we have

$$
\left\|P_{k}\left(g_{k^{\prime}} \cdot h_{k^{\prime \prime}}\right)\right\|_{L_{t}^{\infty} L_{x}^{2}} \lesssim 2^{-\frac{1}{4}\left(\left|k^{\prime}-k\right|+\left|k^{\prime \prime}-k\right|\right)}\left\|g_{k^{\prime}}\right\|_{H^{1}} \cdot\left\|h_{k^{\prime \prime}}\right\|_{H^{1}}
$$


Using the lemma we derive,

$$
\begin{aligned}
\sum_{k}\left\|D_{k}^{2}\right\|_{L_{x}^{2}} & \lesssim \sum_{k} \sum_{k \leq k^{\prime}<k^{\prime \prime}} 2^{k-k^{\prime \prime}}\left\|\partial_{t} g_{k^{\prime \prime}}\right\|_{L_{t}^{2} L_{x}^{2}} \cdot\left\|\nabla h_{k^{\prime}}\right\|_{L_{t}^{2} L_{x}^{2}} \\
& +\sum_{k} \sum_{k \leq k^{\prime}<k^{\prime \prime}} 2^{-\frac{1}{2}\left(\left|k^{\prime}-k\right|+\left|k^{\prime \prime}-k\right|\right)}\left\|g_{k^{\prime \prime}}\right\|_{H^{1}} \cdot\left\|h_{k^{\prime}}\right\|_{H^{1}} \\
& \leq \sum_{k^{\prime}<k^{\prime \prime}} 2^{-\frac{1}{2}\left|k^{\prime}-k^{\prime \prime}\right|}\left\|g_{k^{\prime \prime}}\right\|_{H^{1}} \cdot\left\|h_{k^{\prime}}\right\|_{H^{1}} \lesssim\|g\|_{H^{1}} \cdot\|h\|_{H^{1}}
\end{aligned}
$$

as desired.

3.) Estimates for $B_{k}=P_{k} \int_{0}^{t}\left(\partial_{t} g\right)_{\geq k} \cdot h_{<k}$ : We start by decomposing,

$$
B_{k}=\sum_{k^{\prime}<k \leq k^{\prime \prime}} P_{k} \int_{0}^{t}\left(\partial_{t} g\right)_{k^{\prime \prime}} \cdot h_{k^{\prime}}
$$

Integrating by parts and using lemma 2.2 to estimate the boundary terms, we derive, we obtain, with $J_{k}=\sum_{k^{\prime}<k \leq k^{\prime \prime}} P_{k}\left(g_{k^{\prime \prime}} \cdot h_{k^{\prime}}\right)$,

$$
\begin{aligned}
\left\|B_{k}\right\|_{L_{x}^{2}} & \lesssim \sum_{k^{\prime}<k \leq k^{\prime \prime}}\left\|\int_{0}^{1} g_{k^{\prime \prime}} \cdot\left(\partial_{t} h\right)_{k^{\prime}}\right\|_{L_{x}^{2}}+\left\|J_{k}\right\|_{L_{t}^{\infty} L_{x}^{2}} \\
\left\|J_{k}\right\|_{L_{t}^{\infty} L_{x}^{2}} & \lesssim \sum_{k^{\prime}<k \leq k^{\prime \prime}} 2^{-\frac{1}{2}\left(\left|k^{\prime}-k\right|+\left|k^{\prime \prime}-k\right|\right)}\left\|g_{k^{\prime \prime}}\right\|_{H^{1}} \cdot\left\|h_{k^{\prime}}\right\|_{H^{1}}
\end{aligned}
$$

Now,

$$
\begin{aligned}
\sum_{k^{\prime}<k \leq k^{\prime \prime}}\left\|\int_{0}^{1} g_{k^{\prime \prime}} \cdot\left(\partial_{t} h\right)_{k^{\prime}}\right\|_{L_{x}^{2}} & \lesssim \sum_{k^{\prime}<k \leq k^{\prime \prime}}\left\|g_{k^{\prime \prime}}\right\|_{L_{t}^{2} L_{x}^{2}} \cdot\left\|\left(\partial_{t} h\right)_{k^{\prime}}\right\|_{L_{t}^{2} L_{x}^{\infty}} \\
& \lesssim \sum_{k^{\prime}<k \leq k^{\prime \prime}} 2^{k^{\prime}-k^{\prime \prime}}\left\|\nabla g_{k^{\prime \prime}}\right\|_{L_{t}^{2} L_{x}^{2}} \cdot\left\|\left(\partial_{t} h\right)_{k^{\prime}}\right\|_{L_{t}^{2} L_{x}^{2}}
\end{aligned}
$$

Thus, summing as before,

$$
\begin{aligned}
\sum_{k}\left\|B_{k}\right\|_{L_{x}^{2}} & \lesssim \sum_{k} \sum_{k^{\prime}<k \leq k^{\prime \prime}} 2^{k^{\prime}-k^{\prime \prime}}\left\|\nabla g_{k^{\prime \prime}}\right\|_{L_{t}^{2} L_{x}^{2}} \cdot\left\|\left(\partial_{t} h\right)_{k^{\prime}}\right\|_{L_{t}^{2} L_{x}^{2}} \\
& +\sum_{k} \sum_{k^{\prime}<k \leq k^{\prime \prime}} 2^{-\frac{1}{2}\left(\left|k^{\prime}-k\right|+\left|k^{\prime \prime}-k\right|\right)}\left\|g_{k^{\prime \prime}}\right\|_{H^{1}} \cdot\left\|h_{k^{\prime}}\right\|_{H^{1}} \\
& \lesssim \sum_{k^{\prime}<k^{\prime \prime}} 2^{-\frac{1}{2}\left|k^{\prime}-k^{\prime \prime}\right|}\left\|g_{k^{\prime \prime}}\right\|_{H^{1}} \cdot\left\|h_{k^{\prime}}\right\|_{H^{1}} \lesssim\|g\|_{H^{1}} \cdot\|h\|_{H^{1}}
\end{aligned}
$$

as desired.

Proof of lemma 2.2: By symmetry it suffices to consider the following cases:

$$
k^{\prime} \geq k^{\prime \prime} \geq k, \quad k^{\prime} \geq k>k^{\prime \prime}, \quad k>k^{\prime} \geq k^{\prime \prime}
$$


Observe that the last case, which we call "low-low" interaction, cannot occur. We are thus left with only two cases:

Case 1. $k^{\prime} \geq k^{\prime \prime} \geq k$

$$
\left\|P_{k}\left(g_{k^{\prime}} \cdot h_{k^{\prime \prime}}\right)\right\|_{L_{t}^{\infty} L_{x}^{2}} \lesssim 2^{k}\left\|g_{k^{\prime}} \cdot h_{k^{\prime \prime}}\right\|_{L_{t}^{\infty} L_{x}^{1}} \lesssim 2^{k}\left\|g_{k^{\prime}}\right\|_{L_{t}^{\infty} L_{x}^{2}}\left\|h_{k^{\prime \prime}}\right\|_{L_{t}^{\infty} L_{x}^{2}}
$$

We now make use of the simple calculus inequality,

$$
\|f\|_{L_{t}^{\infty} L_{x}^{2}} \lesssim\left\|\partial_{t} f\right\|_{L_{t}^{2} L_{x}^{2}}^{\frac{1}{2}} \cdot\|f\|_{L_{t}^{2} L_{x}^{2}}^{\frac{1}{2}}+\|f\|_{L_{t}^{2} L_{x}^{2}} .
$$

Thus,

$$
\begin{aligned}
\left\|g_{k^{\prime}}\right\|_{L_{t}^{\infty} L_{x}^{2}} & \lesssim\left\|\partial_{t} g_{k^{\prime}}\right\|_{L_{t}^{2} L_{x}^{2}}^{\frac{1}{2}} \cdot\left\|g_{k^{\prime}}\right\|_{L_{t}^{2} L_{x}^{2}}^{\frac{1}{2}}+\left\|g_{k^{\prime}}\right\|_{L_{t}^{2} L_{x}^{2}} \\
& \lesssim 2^{k^{\prime} / 2}\left(\left\|\partial_{t} g_{k^{\prime}}\right\|_{L_{t}^{2} L_{x}^{2}}^{\frac{1}{2}} \cdot\left\|\nabla g_{k^{\prime}}\right\|_{L_{t}^{2} L_{x}^{2}}^{\frac{1}{2}}+\left\|g_{k^{\prime}}\right\|_{L_{t}^{2} L_{x}^{2}}^{\frac{1}{2}} \cdot\left\|\nabla g_{k^{\prime}}\right\|_{L_{t}^{2} L_{x}^{2}}^{\frac{1}{2}}\right) \\
& \lesssim 2^{k^{\prime} / 2}\left\|\nabla g_{k^{\prime}}\right\|_{L_{t}^{2} L_{x}^{2}}^{\frac{1}{2}}\left(\left\|\partial_{t} g_{k^{\prime}}\right\|_{L_{t}^{2} L_{x}^{2}}+\left\|g_{k^{\prime}}\right\|_{L_{t}^{2} L_{x}^{2}}\right)^{\frac{1}{2}} \\
& \lesssim 2^{k^{\prime} / 2}\left\|g_{k^{\prime}}\right\|_{H^{1}}^{\frac{1}{2}} \cdot\left\|g_{k^{\prime}}\right\|_{H^{1}}^{\frac{1}{2}}=2^{k^{\prime} / 2}\left\|g_{k^{\prime}}\right\|_{H^{1}}
\end{aligned}
$$

Thus,

$$
\left\|g_{k^{\prime}}\right\|_{L_{t}^{\infty} L_{x}^{2}} \lesssim 2^{k^{\prime} / 2}\left\|g_{k^{\prime}}\right\|_{H^{1}}, \quad\left\|h_{k^{\prime \prime}}\right\|_{L_{t}^{\infty} L_{x}^{2}} \lesssim 2^{k^{\prime \prime} / 2}\left\|h_{k^{\prime \prime}}\right\|_{H^{1}}
$$

Therefore, since $k^{\prime} \geq k^{\prime \prime} \geq k$,

$$
\begin{aligned}
\left\|P_{k}\left(g_{k^{\prime}} \cdot h_{k^{\prime \prime}}\right)\right\|_{L_{t}^{\infty} L_{x}^{2}} & \lesssim 2^{k-\frac{k^{\prime}}{2}-\frac{k^{\prime \prime}}{2}}\left\|g_{k^{\prime}}\right\|_{H^{1}} \cdot\left\|h_{k^{\prime \prime}}\right\|_{H^{1}} \\
& =2^{-\frac{1}{2}\left(\left|k^{\prime}-k\right|+\left|k^{\prime \prime}-k\right|\right)}\left\|g_{k^{\prime}}\right\|_{H^{1}} \cdot\left\|h_{k^{\prime \prime}}\right\|_{H^{1}}
\end{aligned}
$$

as desired.

Case 2. $\quad k^{\prime} \geq k>k^{\prime \prime} \quad$ Using once more the estimates (14),

$$
\begin{aligned}
\left\|P_{k}\left(g_{k^{\prime}} \cdot h_{k^{\prime \prime}}\right)\right\|_{L_{t}^{\infty} L_{x}^{2}} & \lesssim\left\|g_{k^{\prime}}\right\|_{L_{t}^{\infty} L_{x}^{2}} \cdot\left\|h_{k^{\prime \prime}}\right\|_{L_{t}^{\infty} L_{x}^{\infty}} \lesssim 2^{k^{\prime \prime}}\left\|g_{k^{\prime}}\right\|_{L_{t}^{\infty} L_{x}^{2}} \cdot\left\|h_{k^{\prime \prime}}\right\|_{L_{t}^{\infty} L_{x}^{2}} \\
& \lesssim 2^{k^{\prime \prime}} 2^{-\frac{k^{\prime}}{2}-\frac{k^{\prime \prime}}{2}}\left\|g_{k^{\prime}}\right\|_{H^{1}} \cdot\left\|h_{k^{\prime \prime}}\right\|_{H^{1}}=2^{\left(k^{\prime \prime}-k^{\prime}\right) / 2}\left\|g_{k^{\prime}}\right\|_{H^{1}} \cdot\left\|h_{k^{\prime \prime}}\right\|_{H^{1}} \\
& =2^{-\frac{1}{2}\left(\left|k^{\prime}-k\right|+\left|k^{\prime \prime}-k\right|\right)}\left\|g_{k^{\prime}}\right\|_{H^{1}} \cdot\left\|h_{k^{\prime \prime}}\right\|_{H^{1}}
\end{aligned}
$$

as desired.

\subsection{Proof of proposition 1.3.}

Proof of (4): As before we have to estimate the sum,

$$
\sum_{k}\left\|P_{k} \int_{0}^{1} g \cdot h d t\right\|_{L_{x}^{2}}
$$

For each integer $k$ we decompose, $h=h_{<k}+h_{\geq k}$ with $h_{<k}=\sum_{k^{\prime}<k} h_{k^{\prime}}$, and $h_{\geq k}=\sum_{k^{\prime} \geq k} h_{k^{\prime}}$. Thus,

$$
P_{k} \int_{0}^{1} g \cdot h d t=P_{k}\left(\int_{0}^{1} g \cdot h_{\geq k}\right)+P_{k}\left(\int_{0}^{1} g \cdot h_{<k}\right)=A_{k}+B_{k}
$$


1.) Estimates for $B_{k}$. Observe that ${ }^{3} P_{k}\left(g \cdot h_{<k}\right)=P_{k}\left(g_{\geq k} \cdot h_{<k}\right)$. Thus, using freely LP5, $B_{k}=P_{k}\left(\int_{0}^{1} g_{\geq k} \cdot h_{<k}\right)$. We now rely on the Bernstein and finite band inequalities LP3, LP4,

$$
\begin{aligned}
\left\|B_{k}\right\|_{L_{x}^{2}} & \lesssim\left\|\int_{0}^{1} g_{\geq k} \cdot h_{<k}\right\|_{L_{x}^{2}} \leq\left\|g_{\geq k}\right\|_{L_{t}^{2} L_{x}^{2}} \cdot\left\|h_{<k}\right\|_{L_{t}^{2} L_{x}^{2}} \\
& \lesssim 2^{k} \sum_{k^{\prime \prime}<k \leq k^{\prime}}\left\|g_{k^{\prime}}\right\|_{L_{t}^{2} L_{x}^{2}} \cdot\left\|h_{k^{\prime \prime}}\right\|_{L_{t}^{2} L_{x}^{\infty}} \\
& \lesssim \sum_{k^{\prime \prime}<k \leq k^{\prime}} 2^{k^{\prime \prime}-k^{\prime}}\left\|\nabla g_{k^{\prime}}\right\|_{L_{t}^{2} L_{x}^{2}} \cdot\left\|h_{k^{\prime \prime}}\right\|_{L_{t}^{2} L_{x}^{2}}
\end{aligned}
$$

Therefore,

$$
\begin{aligned}
\sum_{k}\left\|B_{k}\right\|_{L_{x}^{2}} & \lesssim \sum_{k} \sum_{k^{\prime \prime}<k \leq k^{\prime}} 2^{k^{\prime \prime}-k^{\prime}}\left\|\nabla g_{k^{\prime}}\right\|_{L_{t}^{2} L_{x}^{2}} \cdot\left\|h_{k^{\prime \prime}}\right\|_{L_{t}^{2} L_{x}^{2}} \\
& \lesssim \sum_{k^{\prime \prime} \leq k^{\prime}} 2^{\frac{k^{\prime \prime}-k^{\prime}}{2}}\left\|\nabla g_{k^{\prime}}\right\|_{L_{t}^{2} L_{x}^{2}} \cdot\left\|h_{k^{\prime \prime}}\right\|_{L_{t}^{2} L_{x}^{2}} \lesssim\|\nabla g\|_{L_{t}^{2} L_{x}^{2}} \cdot\|h\|_{L_{t}^{2} L_{x}^{2}} .
\end{aligned}
$$

2.) Estimates for $A_{k}$. To estimate $A_{k}$ we have to be more careful. According 4 to LP3, $\Delta P_{k} f=2{ }^{2 k} P_{k} f$

$$
\begin{aligned}
\left\|A_{k}\right\|_{L_{x}^{2}} & =\left\|P_{k}\left(\int_{0}^{1} g \cdot \Delta h_{\geq k}\right)\right\|_{L_{x}^{2}} \lesssim \sum_{k^{\prime} \geq k} 2^{-2 k^{\prime}}\left\|P_{k}\left(\int_{0}^{1} g \cdot \Delta h_{k^{\prime}}\right)\right\|_{L_{x}^{2}} \\
& \lesssim \sum_{k^{\prime} \geq k} 2^{-2 k^{\prime}}\left\|P_{k}\left(\nabla \int_{0}^{1}\left(g \cdot \nabla h_{k^{\prime}}\right)\right)\right\|_{L_{x}^{2}}+\sum_{k^{\prime} \geq k} 2^{-2 k^{\prime}}\left\|P_{k}\left(\int_{0}^{1} \nabla g \cdot \nabla h_{k^{\prime}}\right)\right\|_{L_{x}^{2}} \\
& =J_{1}+J_{2}
\end{aligned}
$$

Now, using LP3,

$$
\begin{aligned}
J_{1} & =\sum_{k^{\prime} \geq k} 2^{-2 k^{\prime}}\left\|P_{k}\left(\nabla \int_{0}^{1}\left(g \cdot \nabla h_{k^{\prime}}\right)\right)\right\|_{L_{x}^{2}} \lesssim \sum_{k^{\prime} \geq k} 2^{-2 k^{\prime}+k}\left\|\int_{0}^{1} g \cdot \nabla h_{k^{\prime}}\right\|_{L_{x}^{2}} \\
& \lesssim \sum_{k^{\prime} \geq k} 2^{-2 k^{\prime}+k}\left\|\left(\int_{0}^{1}|g|^{2}\right)^{\frac{1}{2}} \cdot\left(\int_{0}^{1}\left|\nabla h_{k^{\prime}}\right|^{2}\right)^{\frac{1}{2}}\right\|_{L_{x}^{2}} \\
& \lesssim \sum_{k^{\prime} \geq k} 2^{-2 k^{\prime}+k}\|g\|_{L_{x}^{\infty} L_{t}^{2}} \cdot\left\|\nabla h_{k^{\prime}}\right\|_{L_{t}^{2} L_{x}^{2}} \lesssim \sum_{k^{\prime} \geq k} 2^{-k^{\prime}+k}\|g\|_{L_{x}^{\infty} L_{t}^{2}} \cdot\left\|h_{k^{\prime}}\right\|_{L_{t}^{2} L_{x}^{2}}
\end{aligned}
$$

On the other hand, using the dual Bernstein inequality

$$
\begin{aligned}
J_{2} & =\sum_{k^{\prime} \geq k} 2^{-2 k^{\prime}}\left\|P_{k}\left(\int_{0}^{1} \nabla g \cdot \nabla h_{k^{\prime}}\right)\right\|_{L_{x}^{2}} \lesssim \sum_{k^{\prime} \geq k} 2^{-2 k^{\prime}+k}\left\|\left(\int_{0}^{1} \nabla g \cdot \nabla h_{k^{\prime}}\right)\right\|_{L_{x}^{1}} \\
& \lesssim \sum_{k^{\prime} \geq k} 2^{-2 k^{\prime}+k}\|\nabla g\|_{L_{t}^{2} L_{x}^{2}} \cdot\left\|\nabla h_{k^{\prime}}\right\|_{L_{t}^{2} L_{x}^{2}} \lesssim \sum_{k^{\prime} \geq k} 2^{-k^{\prime}+k}\|\nabla g\|_{L_{t}^{2} L_{x}^{2}} \cdot\left\|h_{k^{\prime}}\right\|_{L_{t}^{2} L_{x}^{2}}
\end{aligned}
$$

Hence,

$$
\left\|A_{k}\right\|_{L_{x}^{2}} \lesssim \sum_{k^{\prime} \geq k} 2^{-k^{\prime}+k}\left\|h_{k^{\prime}}\right\|_{L_{t}^{2} L_{x}^{2}} \cdot\left(\|\nabla g\|_{L_{t}^{2} L_{x}^{2}}+\|g\|_{L_{x}^{\infty} L_{t}^{2}}\right)
$$

\footnotetext{
${ }^{3}$ In fact $P_{k}\left(g \cdot h_{<k}\right)=P_{k}\left(g_{>k+1} \cdot h_{<k}\right)$, neglecting a finite number of terms does not matter.

${ }^{4}$ In fact $\Delta P_{k} f=2^{2 k} \tilde{P}_{k} f$ with a slightly modified LP -projection.
} 
and therefore,

$$
\begin{aligned}
\sum_{k}\left\|A_{k}\right\|_{L_{x}^{2}} & \lesssim \sum_{k^{\prime}}\left\|h_{k^{\prime}}\right\|_{L_{t}^{2} L_{x}^{2}} \cdot\left(\|\nabla g\|_{L_{t}^{2} L_{x}^{2}}+\|g\|_{L_{x}^{\infty} L_{t}^{2}}\right) \\
& \lesssim\left(\|\nabla g\|_{L_{t}^{2} L_{x}^{2}}+\|g\|_{L_{x}^{\infty} L_{t}^{2}}\right) \cdot\|h\|_{L_{t}^{2} B_{x}^{0}}
\end{aligned}
$$

as desired.

Proof of (5): We have to estimate the sum,

$$
\sum_{k}\left\|P_{k}\left(g \cdot \int_{0}^{t} h\right)\right\|_{L_{t}^{2} L_{x}^{2}}
$$

For each integer $k$ we decompose, $h=h_{<k}+h_{\geq k}$ with $h_{<k}=\sum_{k^{\prime}<k} h_{k^{\prime}}$, and $h_{\geq k}=\sum_{k^{\prime} \geq k} h_{k^{\prime}}$. Thus,

$$
P_{k}\left(g \cdot \int_{0}^{t} h\right) d t=P_{k}\left(g \cdot \int_{0}^{t} h_{\geq k}\right)+P_{k}\left(g \cdot \int_{0}^{t} h_{<k}\right)=A_{k}+B_{k}
$$

1.) Estimates for $B_{k}$. Observe that ${ }^{5} P_{k}\left(g \cdot \int_{0}^{t} h_{<k}\right)=P_{k}\left(g_{\geq k} \cdot \int_{0}^{t} h_{<k}\right)$. Thus, using freely LP5, $B_{k}=P_{k}\left(g_{\geq k} \cdot \int_{0}^{t} h_{<k}\right)$. We rely on LP3, LP4,

$$
\begin{aligned}
\left\|B_{k}\right\|_{L_{t}^{2} L_{x}^{2}} & \lesssim\left\|g_{\geq k} \cdot \int_{0}^{t} h_{<k}\right\|_{L_{t}^{2} L_{x}^{2}} \leq\left\|g_{\geq k}\right\|_{L_{t}^{2} L_{x}^{2}} \cdot\left\|\int_{0}^{t} h_{<k}\right\|_{L_{t}^{\infty} L_{x}^{2}} \\
& \lesssim 2^{k} \sum_{k^{\prime \prime}<k \leq k^{\prime}}\left\|g_{k^{\prime}}\right\|_{L_{t}^{2} L_{x}^{2}} \cdot\left\|h_{k^{\prime \prime}}\right\|_{L_{t}^{1} L_{x}^{\infty}} \\
& \lesssim \sum_{k^{\prime \prime}<k \leq k^{\prime}} 2^{k^{\prime \prime}-k^{\prime}}\left\|\nabla g_{k^{\prime}}\right\|_{L_{t}^{2} L_{x}^{2}} \cdot\left\|h_{k^{\prime \prime}}\right\|_{L_{t}^{1} L_{x}^{2}}
\end{aligned}
$$

Therefore,

$$
\begin{aligned}
\sum_{k}\left\|B_{k}\right\|_{L_{t}^{2} L_{x}^{2}} & \lesssim \sum_{k} \sum_{k^{\prime \prime}<k \leq k^{\prime}} 2^{k^{\prime \prime}-k^{\prime}}\left\|\nabla g_{k^{\prime}}\right\|_{L_{t}^{2} L_{x}^{2}} \cdot\left\|h_{k^{\prime \prime}}\right\|_{L_{t}^{1} L_{x}^{2}} \\
& \lesssim \sum_{k^{\prime \prime} \leq k^{\prime}} 2^{\frac{k^{\prime \prime}-k^{\prime}}{2}}\left\|\nabla g_{k^{\prime}}\right\|_{L_{t}^{2} L_{x}^{2}} \cdot\left\|h_{k^{\prime \prime}}\right\|_{L_{t}^{1} L_{x}^{2}} \lesssim\|\nabla g\|_{L_{t}^{2} L_{x}^{2}} \cdot\|h\|_{L_{t}^{1} L_{x}^{2}}
\end{aligned}
$$

2.) Estimates for $A_{k}$. To estimate $A_{k}$ we proceed as in the proof of (4).

$$
\begin{aligned}
\left\|A_{k}\right\|_{L_{t}^{2} L_{x}^{2}} & =\left\|P_{k}\left(g \cdot \int_{0}^{t} h_{\geq k}\right)\right\|_{L_{t}^{2} L_{x}^{2}} \lesssim \sum_{k^{\prime} \geq k} 2^{-2 k^{\prime}}\left\|P_{k}\left(g \cdot \int_{0}^{t} \Delta h_{k^{\prime}}\right)\right\|_{L_{t}^{2} L_{x}^{2}} \\
& \lesssim \sum_{k^{\prime} \geq k} 2^{-2 k^{\prime}}\left\|P_{k}\left(\nabla\left(g \cdot \int_{0}^{t} \nabla h_{k^{\prime}}\right)\right)\right\|_{L_{t}^{2} L_{x}^{2}} \\
& +\sum_{k^{\prime} \geq k} 2^{-2 k^{\prime}}\left\|P_{k}\left(\nabla g \cdot \int_{0}^{t} \nabla h_{k^{\prime}}\right)\right\|_{L_{t}^{2} L_{x}^{2}} \\
& =J_{1}+J_{2}
\end{aligned}
$$

\footnotetext{
${ }^{5}$ In fact $P_{k}\left(g \cdot h_{<k}\right)=P_{k}\left(g_{>k+1} \cdot h_{<k}\right)$, neglecting a finite number of terms does not matter.
} 
Now, using LP3,

$$
\begin{aligned}
J_{1} & =\sum_{k^{\prime} \geq k} 2^{-2 k^{\prime}}\left\|P_{k}\left(\nabla\left(g \cdot \int_{0}^{t} \nabla h_{k^{\prime}}\right)\right)\right\|_{L_{t}^{2} L_{x}^{2}} \lesssim \sum_{k^{\prime} \geq k} 2^{-2 k^{\prime}+k}\left\|g \cdot \int_{0}^{t} \nabla h_{k^{\prime}}\right\|_{L_{t}^{2} L_{x}^{2}} \\
& \lesssim \sum_{k^{\prime} \geq k} 2^{-2 k^{\prime}+k}\|g\|_{L_{x}^{\infty} L_{t}^{2}} \cdot\left\|\int_{0}^{t} \nabla h_{k^{\prime}}\right\|_{L_{x}^{2} L_{t}^{\infty}} \lesssim \sum_{k^{\prime} \geq k} 2^{-k^{\prime}+k}\|g\|_{L_{x}^{\infty} L_{t}^{2}} \cdot\left\|h_{k^{\prime}}\right\|_{L_{t}^{1} L_{x}^{2}}
\end{aligned}
$$

On the other hand, using the dual Bernstein inequality

$$
\begin{aligned}
J_{2} & =\sum_{k^{\prime} \geq k} 2^{-2 k^{\prime}}\left\|P_{k}\left(\nabla g \cdot \int_{0}^{t} \nabla h_{k^{\prime}}\right)\right\|_{L_{t}^{2} L_{x}^{2}} \lesssim \sum_{k^{\prime} \geq k} 2^{-2 k^{\prime}+k}\left\|\nabla g \cdot \int_{0}^{t} \nabla h_{k^{\prime}}\right\|_{L_{t}^{2} L_{x}^{1}} \\
& \lesssim \sum_{k^{\prime} \geq k} 2^{-2 k^{\prime}+k}\|\nabla g\|_{L_{t}^{2} L_{x}^{2}} \cdot\left\|\int_{0}^{t} \nabla h_{k^{\prime}}\right\|_{L_{t}^{\infty} L_{x}^{2}} \\
& \lesssim \sum_{k^{\prime} \geq k} 2^{-k^{\prime}+k}\|\nabla g\|_{L_{t}^{2} L_{x}^{2}} \cdot\left\|h_{k^{\prime}}\right\|_{L_{t}^{1} L_{x}^{2}}
\end{aligned}
$$

Hence,

$$
\left\|A_{k}\right\|_{L_{t}^{2} L_{x}^{2}} \lesssim \sum_{k^{\prime} \geq k} 2^{-k^{\prime}+k}\left\|h_{k^{\prime}}\right\|_{L_{t}^{1} L_{x}^{2}} \cdot\left(\|\nabla g\|_{L_{t}^{2} L_{x}^{2}}+\|g\|_{L_{x}^{\infty} L_{t}^{2}}\right)
$$

and therefore,

$$
\begin{aligned}
\sum_{k}\left\|A_{k}\right\|_{L_{t}^{2} L_{x}^{2}} & \lesssim \sum_{k^{\prime}}\left\|h_{k^{\prime}}\right\|_{L_{t}^{1} L_{x}^{2}} \cdot\left(\|\nabla g\|_{L_{t}^{2} L_{x}^{2}}+\|g\|_{L_{x}^{\infty} L_{t}^{2}}\right) \\
& \lesssim\left(\|\nabla g\|_{L_{t}^{2} L_{x}^{2}}+\|g\|_{L_{x}^{\infty} L_{t}^{2}}\right) \cdot\|h\|_{L_{t}^{1} B_{x}^{0}}
\end{aligned}
$$

as desired.

\section{Geometric Set-up. Geometric LP- projections}

We assume given an Einstein spacetime $(\mathcal{M}, \mathbf{g})$ a space -like hypersurface $\Sigma$ and an outgoing null hypersurface $\mathcal{H}$, initiating on a compact 2 surface $S_{0} \subset \Sigma$ diffeomorphic to $\mathbb{S}^{2}$, given by the level hypersurfaces of an optical function $u$, i.e. solution to the Eikonal equation

$$
\mathbf{g}^{\alpha \beta} \partial_{\alpha} u \partial_{\beta} u=0 .
$$

We briefly recall the main geometric definitions, see section 2 of [Kl-Rodn1], associated with $\mathcal{H}$.

1.) Geodesic foliation: Let $L=-\mathbf{g}^{\alpha \beta} \partial_{a} u \partial_{b}$ be the corresponding null generator vectorfield and $s$ its affine parameter, i.e. $L(s)=1,\left.\quad s\right|_{S_{0}}=0$. The level surfaces $S_{s}$ of $s$ generates the geodesic foliation on $\mathcal{H}$. We shall denote by $\nabla$ the covariant differentiation on $S_{s}$ and by $\nabla_{L}$ the projection to $S_{s}$ of the covariant derivative with respect to $L$, see section 2. We also denote by $r$ the function on $\mathcal{H}$ defined by $r=r(s)=\sqrt{(4 \pi)^{-1}\left|S_{s}\right|}$, with $\left|S_{s}\right|$ the area of $S_{s}$. Let $\mathcal{H}_{t}$ be the portion of $\mathcal{H}$ between $s=0$ and $s=t$ and, for simplicity, assume $\mathcal{H}=\mathcal{H}_{1}$. 
2.) Null pair: With our choice of $L$ we have

$$
<L, L>=0, \quad D_{L} L=0
$$

where $<,>=<,>_{g}$ denoting the metric of $\mathcal{M}$. At any point $P \in S_{s} \subset \mathcal{H}$ we denote by $\underline{L}$ the null vector conjugate to $L$ relative to the geodesic foliation, i.e.

$$
<L, \underline{L}>=-2, \quad<\underline{L}, X>=0 \quad \text { for all } X \in T_{p}\left(S_{s}\right) .
$$

We shall say that $L, \underline{L}$ form the canonical null pair associated to the foliation. We denote by $\gamma$ the induced metric on $S_{v}$, by $\nabla$ the induced covariant derivative and $K$ the Gauss curvature. An arbitrary orthonormal frame on $S_{v}$ will be denoted by $\left(e_{a}\right)_{a=1,2}$. Clearly,

$$
<e_{a}, L>=<e_{a}, \underline{L}>=0, \quad<e_{a}, e_{b}>=\delta_{a b} .
$$

A null pair together with an orthonormal frame $\left(e_{a}\right)_{a=1,2}$ as above is called a null frame associated to the foliation.

3.) Total curvature flux: We introduce the total curvature flux along $\mathcal{H}$ to be the integral ${ }^{6}$, see precise definition of the null curvature components $\alpha, \beta, \rho, \sigma, \beta$ in section 2 of [Kl-Rodn1],

$$
\mathcal{R}_{0}=\left(\|\alpha\|_{L^{2}(\mathcal{H})}^{2}+\|\beta\|_{L^{2}(\mathcal{H})}^{2}+\|\rho\|_{L^{2}(\mathcal{H})}^{2}+\|\sigma\|_{L^{2}(\mathcal{H})}^{2}+\|\underline{\beta}\|_{L^{2}(\mathcal{H})}^{2}\right)^{\frac{1}{2}}
$$

with $\alpha, \beta, \rho, \sigma, \underline{\beta}$ null components of the curvature tensor $\mathbf{R}$ of the spacetime, background metric $\overline{\mathbf{g}}$.

In [Kl-Rodn1] we worked under the assumption the $\mathcal{R}_{0}$ is sufficiently small. In this paper the only curvature components we shall need are $\beta$ and the Gauss curvature $K$ of the $S_{s}$ surfaces. We will make specific assumptions about this, consistent with the small curvature flux condition, i.e. $\mathcal{R}_{0}$ sufficiently small.

4.) Null connection coefficients: The null second fundamental forms $\chi, \underline{\chi}$ of the foliation $S_{v}$ are given by

$$
\chi_{a b}=<D_{a} L, e_{b}>, \quad \underline{\chi}_{a b}=<D_{a} \underline{L}, e_{b}>
$$

The torsion is given by,

$$
\zeta_{a}=\frac{1}{2}<D_{a} L, \underline{L}>
$$

We also denote $\operatorname{tr} \chi=\delta^{a b} \chi_{a b}$ and $\operatorname{tr} \underline{\chi}=\delta^{a b} \underline{\hat{\chi}}_{a b}$ and $\hat{\chi}=\hat{\chi}-\frac{1}{2} \operatorname{tr} \chi \delta, \underline{\hat{\chi}}=\underline{\chi}-\frac{1}{2} \operatorname{tr} \underline{\chi} \delta$. Recall the definition of the mass aspect function $\mu=-\operatorname{div} \zeta+\frac{1}{2} \hat{\chi} \cdot \underline{\hat{\chi}}-\bar{\rho}+|\zeta|^{2}$.

5.) Commutator formulas Commutation formulas between $\nabla_{L}$ and $\nabla$ play an important role in the paper. We recall, see section 2.15 of [Kl-Rodn1],

\footnotetext{
${ }^{6}$ Here $\|F\|_{L^{2}(\mathcal{H})}=\left(\int_{0}^{1} d s \int_{S_{s}}|F|^{2}\right)^{\frac{1}{2}}$ where $\int_{S_{s}}|F|^{2}$ denotes the integral with respect to the volume element $d \mu_{s}$ of $S_{s}$.
} 
Proposition 3.1. Consider an arbitrary $k$-covariant, $S$-tangent vectorfield $F_{\underline{a}}=$ $F_{a_{1} \ldots a_{k}}$ Then,

$$
\nabla_{L} \nabla_{b} F_{\underline{a}}-\nabla_{b} \nabla_{L} F_{\underline{a}}=-\chi_{b c} \nabla_{c} F_{\underline{a}}+\sum_{i}\left(\chi_{a_{i} b} \zeta_{c}-\chi_{b c} \zeta_{a_{i}}+\epsilon_{a_{i} c}{ }^{\star} \beta_{b}\right) F_{a_{1} \ldots c \ldots a_{k}}
$$

In particular for scalars $f$,

$$
\nabla_{L} \nabla_{b} f-\nabla_{b} \nabla_{L} f=-\chi_{b c} \nabla_{c} f
$$

Also, for a one form $F$,

$$
L(\operatorname{div} F)-\operatorname{div}\left(\nabla_{L} F\right)=-\chi \cdot \nabla F+\left(\frac{1}{2} \operatorname{tr} \chi \cdot \zeta+\hat{\chi} \cdot \zeta-\beta\right) \cdot F
$$

and again for scalars,

$$
L(\Delta f)-\Delta(L f)=-\operatorname{tr} \chi \Delta f-2 \hat{\chi} \cdot \nabla^{2} f+(\nabla \cdot \operatorname{tr} \chi+2 \hat{\chi} \cdot \zeta+\operatorname{tr} \chi \cdot \zeta) \cdot \nabla f
$$

6.) Bochner identity Bochner identity holds for scalars and tensors on surfaces $S=S_{s}, 0 \leq s \leq 1$,

\section{Proposition 3.2.}

i) For a scalar function $f$,

$$
\int_{S}\left|\nabla^{2} f\right|^{2}=\int_{S}|\Delta f|^{2}-\int_{S} K|\nabla f|^{2}
$$

ii) For a tensorfield $F$

$$
\int_{S}\left|\nabla^{2} F\right|^{2}=\int_{S}|\Delta F|^{2}-\int_{S} K\left(2|\nabla F|^{2}-|\operatorname{div} F|^{2}\right)+\int_{S} K^{2}|F|^{2}
$$

Remark 3.3. The difference between scalars and tensors is substantial as terms quadratic in the curvature are much more difficult to control, see the properties K1-K2 we state in the next section.

3.4. Main geometric properties of $\mathcal{H}$. The proof of the Main Theorem of [Kl-Rodn1] was based on the bootstrap assumptions BA1-BA4 concerning the geometric quantities $\operatorname{tr} \chi, \hat{\chi}, \zeta, \operatorname{tr} \chi, \hat{\chi}$. In this paper we make a consistent but somewhat different set of assumptions, A1, A2, WS, K1, K2 concerning tr $\chi, \hat{\chi}$, $\zeta$ and the Gauss curvature $K$. In the following theorem we stress the fact that these assumptions follow from the bootstrap assumptions BA1-BA3, the small curvature flux and initial conditions $\mathcal{R}_{0}, \mathcal{I}_{0}$ of [Kl-Rodn1].

Proposition 3.5. The geometric properties of the geodesic foliation of $\mathcal{H}$ described below in A1, A2, WS, K1, K2 follow from the bootstrap assumptions BA1-BA3, the small curvature flux and the initial conditions $\mathcal{R}_{0}, \mathcal{I}_{0}$ of [Kl-Rodn1] 
Proof : It will become obvious that A1, A2 are contained in assumptions BA1, BA2. Properties WS, K1, K2 have been carefully derived in section 4 of [Kl-Rodn1] as consequences BA1-BA3, the small curvature flux and the initial conditions $\mathcal{R}_{0}$, $\mathcal{I}_{0}$.

We now describe properties A1, A2, WS, K1, K2 and their immediate consequences.

The most primitive assumption is, as in [Kl-Rodn1]:

A1. $\sup _{\mathcal{H}} r\left|\overline{\operatorname{tr} \chi}-\frac{2}{r}\right| \leq \Delta_{0}, \quad \sup _{\mathcal{H}} r|\operatorname{tr} \chi-\overline{\operatorname{tr} \chi}| \leq \Delta_{0} . \quad$ where $0<$ $\Delta_{0}<\frac{1}{2}$ is a sufficiently small constant.

Based on the assumption A1 we could easily deduced, see section 3.7 in [Kl-Rodn1],

$$
r_{0}+\frac{1}{2} s \leq r \leq r_{0}+\frac{3}{2} s .
$$

Moreover,

$$
1 \leq \frac{\sqrt{\mid} \gamma_{s} \mid}{\sqrt{\mid} \gamma_{0} \mid} \leq 2\left(\frac{3}{2}\right)^{6}, \quad \text { for all } \quad 0 \leq s \leq t \leq 1
$$

i.e. the volume elements of $S_{s}$ and $S_{0}$ remain comparable in the interval $0 \leq s \leq 1$. As a consequence of $(22)$ we also infer that the $L^{2}(\mathcal{H})$ norm, defined in the footnote 6 below, is equivalent to the product norm on $[0, t] \times S_{0}$,

$$
\|F\|_{L^{2}}=\|F\|_{L_{t}^{2} L_{x}^{2}}=\left(\int_{0}^{t} \int_{S_{0}}|F|^{2} d s d \mu_{0}\right)^{\frac{1}{2}}=\left(\int_{0}^{t} d s \int_{S_{0}}|F(s, \omega)|^{2} \sqrt{\left|\gamma_{0}\right|} d \omega\right)_{23)}^{\frac{1}{2}}
$$

We shall also make use of the following norms,

$$
\begin{aligned}
& \|F\|_{L_{x}^{\infty} L_{t}^{2}}=\sup _{\omega \in S_{0}}\left(\int_{0}^{t} d s|F(s, \omega)|^{2}\right)^{\frac{1}{2}} \\
& \|F\|_{L_{x}^{2} L_{t}^{\infty}}=\left\|\sup _{0 \leq s \leq 1}|F(s, \omega)|\right\|_{L^{2}\left(S_{0}\right)}
\end{aligned}
$$

as well as, for $1 \leq p \leq \infty$,

$$
\begin{aligned}
\|F\|_{L_{t}^{\infty} L_{x}^{p}} & =\sup _{0 \leq s \leq t}\|F(s)\|_{L^{p}\left(S_{0}\right)} \\
\|F\|_{L_{t}^{2} L_{x}^{p}} & =\left(\int_{0}^{t}\|F(s)\|_{L^{p}\left(S_{0}\right)}^{2} d s\right)^{\frac{1}{2}}
\end{aligned}
$$

Observe that $\|F\|_{L_{t}^{\infty} L_{x}^{2}} \leq\|F\|_{L_{x}^{2} L_{t}^{\infty}}$. We recall the following transport lemma, see section 3.7 in [Kl-Rodn1].

Lemma 3.6. Consider the equation $\nabla_{L} F+k \operatorname{tr} \chi F=G$ for $S$-tangent tensors $F, G$ on $\mathcal{H}$. Then, for any $p \geq 1$,

$$
\|F\|_{L_{x}^{p} L_{t}^{\infty}} \lesssim\|F(0)\|_{L^{p}\left(S_{0}\right)}+\|F\|_{L_{x}^{p} L_{t}^{1}}
$$


We shall also make us of the following notations:

Definition 3.7. Given an arbitrary $S$-tangent tensor $H$ on $\mathcal{H}=\mathcal{H}_{1}$ we denote

$$
\|\bar{\nabla} F\|_{L^{2}}=\|\nabla F\|_{L^{2}}+\left\|\nabla_{L} F\right\|_{L^{2}} .
$$

We also introduce the following norms ,

$$
\begin{aligned}
\mathcal{N}_{1}(F) & =\|F\|_{L^{2}}+\|\bar{\nabla} F\|_{L^{2}} \\
& =\|F\|_{L^{2}}+\|\nabla F\|_{L^{2}}+\left\|\nabla_{L} F\right\|_{L^{2}} \\
\mathcal{N}_{2}(F) & =\|F\|_{L^{2}}+\|\bar{\nabla} F\|_{L^{2}}+\|\nabla \bar{\nabla} F\|_{L^{2}} \\
& =\|F\|_{L^{2}}+\|\nabla F\|_{L^{2}}+\left\|\nabla^{2} F\right\|_{L^{2}} \\
& +\left\|\nabla_{L} F\right\|_{L^{2}}+\left\|\nabla \cdot \nabla_{L} F\right\|_{L^{2}}
\end{aligned}
$$

where $L^{2}$ here stands for $L_{t}^{2} L_{x}^{2}$.

The second set of assumptions we need is:

A2.

$$
\begin{aligned}
\|\hat{\chi}\|_{L_{x}^{\infty} L_{t}^{2}}, \quad\|\zeta\|_{L_{x}^{\infty} L_{t}^{2}} & \leq \Delta_{0}, \\
\|\nabla \operatorname{tr} \chi\|_{L_{x}^{2} L_{t}^{\infty}}, \quad \mathcal{N}_{1}(\hat{\chi}), \quad \mathcal{N}_{1}(\zeta) & \leq \Delta_{0}
\end{aligned}
$$

Remark 3.8. The assumptions A2 are essentially the same as BA2 of [Kl-Rodn1] except for the bound on $\mu$ which is not needed here.

As in [Kl-Rodn1] we can simplify our various calculations by introducing the following symbolic notations for connection coefficients.

Definition 3.9. We denote by $A$ the collection formed by the connection coefficients: $A=\operatorname{tr} \chi-\frac{2}{r}, \hat{\chi}, \zeta$

With these notation the assumptions, $\mathbf{A} \mathbf{1}$ and $\mathbf{A 2}$ take the form,

$$
\left\|\operatorname{tr} \chi-\frac{2}{r}\right\|_{L_{t}^{\infty} L_{x}^{\infty}}, \quad\|\nabla \operatorname{tr} \chi\|_{L_{x}^{2} L_{t}^{\infty}}, \quad\|A\|_{L_{x}^{\infty} L_{t}^{2}}, \quad \mathcal{N}_{1}[A] \lesssim \Delta_{0}
$$

The following inequalities are straightforward consequences of $\mathbf{A 1}$ and A2, see[Kl-Rodn1]:

Lemma 3.10. The following estimates hold for an arbitrary, smooth, S-tangent tensorfield $F$ :

$$
\begin{aligned}
\|F\|_{L_{t}^{\infty} L_{x}^{2}},\|F\|_{L_{t}^{\infty} L_{x}^{4}}, \quad\|F\|_{L_{t}^{6} L_{x}^{6}} & \lesssim \mathcal{N}_{1}[F] \\
\|F\|_{L_{t}^{\infty} L_{x}^{\infty}} & \lesssim \mathcal{N}_{2}[F]
\end{aligned}
$$

Lemma 3.11. Let $w$ be a solution of the scalar transport equation

$$
\nabla_{L} w=f,\left.\quad w\right|_{S_{0}}=0,
$$

For any $p \geq 1$,

$$
\|\nabla w\|_{L_{x}^{p} L_{t}^{\infty}} \lesssim\|\nabla f\|_{L_{x}^{p} L_{t}^{1}}
$$


Proof : We only need to differentiate according to the commutator formula of proposition $3.1,\left[\nabla_{L}, \nabla\right] w=-\frac{1}{2} \operatorname{tr} \chi \cdot \nabla w-\hat{\chi} \cdot \nabla w$ and then apply lemma 3.6 to the transport equation,

$$
\nabla_{L} \nabla w+\frac{1}{2} \operatorname{tr} \chi \cdot \nabla w=\nabla f-\hat{\chi} \cdot \nabla w
$$

The notation introduced in definition 3.9 allows us to express in a compact form the commutator formulas of proposition 3.1 More precisely,

Proposition 3.12. In what follows we denote arbitrary $S$ tangent tensorfields by capital letters $F$ and scalars by low case letters $f$.

$$
\begin{gathered}
{\left[\nabla_{L}, \nabla\right] f=-\frac{1}{2} \operatorname{tr\chi } \cdot \nabla f+A \cdot \nabla f} \\
{\left[\nabla_{L}, \Delta\right] f=\nabla\left(\left(\frac{1}{r}+A\right) \cdot \nabla f\right)+\nabla A \cdot \nabla f+A \cdot A \cdot \nabla f} \\
{\left[\nabla_{L}, \nabla\right] F=-\frac{1}{2} \operatorname{tr} \chi \cdot \nabla F+A \cdot \nabla F+\beta \cdot F+\left(A+\frac{1}{r}\right) \cdot A \cdot F} \\
{\left[\nabla_{L}, \Delta\right] F=-\operatorname{tr\chi } \Delta f+A \cdot \nabla^{2} F+\nabla A \cdot \nabla F+\left(A+\frac{1}{r}\right) \cdot A \cdot \nabla F} \\
+\beta \cdot \nabla F+\nabla\left(\beta \cdot \nabla F+\left(A+\frac{1}{r}\right) \cdot A \cdot F\right)
\end{gathered}
$$

Lemma 3.13. For a given 1-form $F$ let $w$ be a solution of the scalar transport equation

$$
\nabla_{L} w=\operatorname{div} F,\left.\quad w\right|_{S_{0}}=0,
$$

and let 1-form $W$ be a solution of the equation

$$
\nabla_{L} W-\chi \cdot W=F,\left.\quad W\right|_{S_{0}}=0 .
$$

Then for any $1 \leq p \leq 2$,

$$
\|\operatorname{div} W-w\|_{L_{x}^{p} L_{t}^{\infty}} \lesssim \Delta_{0}\|F\|_{L_{x}^{\frac{2 p}{2-p}} L_{t}^{1}} .
$$

Proof: We commute the equation (35) with div, using the commutation formula of proposition 3.12, and subtract the transport equation for $w$ we obtain

$$
\nabla_{L}(\operatorname{div} W-w)=\nabla \chi \cdot W+\beta \cdot W+\left(A+\frac{1}{r}\right) \cdot A \cdot W
$$

Applying the estimate (28) of lemma 3.6 we infer that

$$
\begin{aligned}
\|\operatorname{div} W-w\|_{L_{x}^{p} L_{t}^{\infty}} & \lesssim\|\nabla \chi \cdot W\|_{L_{x}^{p} L_{t}^{1}}+\|\beta \cdot W\|_{L_{x}^{p} L_{t}^{1}}+\left\|\left(A+\frac{1}{r}\right) \cdot A \cdot W\right\|_{L_{x}^{p} L_{t}^{1}} \\
& \lesssim\|A\|_{L_{x}^{\infty} L_{t}^{2}}^{2} \cdot\|W\|_{L_{x}^{p} L_{t}^{\infty}}+\left(\|\beta\|_{L_{t}^{2} L_{x}^{2}}+\|\nabla A\|_{L_{t}^{2} L_{x}^{2}}\right) \cdot\|W\|_{L_{x}^{2-p} L_{t}^{2}}
\end{aligned}
$$


Again applying the estimate (28) of lemma 3.6 to the transport equation for $W$ and using the conditions A1, A2, we derive

$$
\|\operatorname{div} W-w\|_{L_{x}^{p} L_{t}^{\infty}} \lesssim \Delta_{0}\left(\|F\|_{L_{x}^{\frac{2 p}{2-p}} L_{t}^{1}}+\Delta_{0}\|F\|_{L_{x}^{p} L_{t}^{1}}\right) \lesssim \Delta_{0}\|F\|_{L_{x}^{\frac{2 p}{2-p}} L_{t}^{1}}
$$

as desired.

In addition to $\mathbf{A} \mathbf{1}-\mathbf{A} \mathbf{2}$ we need two other type of assumptions.

WS. The initial surface $S_{0}$ can be covered with a finite number of coordinate charts $\left(w^{1}, w^{2}\right)$ such that relative to the transported coordinates $\left(s, \omega^{a}\right)$ on $\mathcal{H}$, with $s$ the afine parameter, the metric $\gamma$ and its partial derivatives $\partial \gamma$, relative to the coordinates $(s, \omega)$, verify the estimates

$$
\|\gamma\|_{L_{t}^{\infty} L_{x}^{\infty}}, \quad\left\|\gamma^{-1}\right\|_{L_{t}^{\infty} L_{x}^{\infty}} \lesssim 1, \quad\left\|\partial\left(\gamma-\stackrel{\circ}{\gamma}_{s}\right)\right\|_{L_{x}^{2} L_{t}^{\infty}} \lesssim \Delta_{0}
$$

where $\stackrel{\circ}{\gamma}_{s}=(1+s)^{2} \stackrel{\circ}{\gamma}$ and $\stackrel{\circ}{\gamma}$ denotes the standard metric on $S$, isometric to that of $\mathbb{S}^{2}$. We shall also make assumptions on the Gauss curvature $K$ of the surfaces $S_{s}$.

K1. The Gauss curvature $K$ of the $S_{s}$ surfaces and the null curvature component $\beta$, see (16), verify:

$$
\left\|K-\frac{1}{r^{2}}\right\|_{L_{t}^{2} L_{x}^{2}}, \quad\|\beta\|_{L_{t}^{2} L_{x}^{2}} \lesssim \Delta_{0}
$$

K2. The Gauss curvature $K$ of the $S_{s}$ surfaces satisfies

$$
\left\|\Lambda^{-\gamma}\left(K-r^{-2}\right)\right\|_{L_{x}^{2} L_{t}^{\infty}} \lesssim \Delta_{0}
$$

with $\Lambda^{-\gamma}=(1-\Delta)^{-\gamma / 2}$, for any $\gamma>\frac{1}{2}$.

The properties WS, K1, K2 allow us to apply all the results of [Kl-Rodn2]. In what follows we shall present a summary of the results proved in [Kl-Rodn2] which shall be needed in this paper.

\subsection{Calculus inequalities on surfaces.}

Proposition 3.15. The following calculus inequalities hold true for our surfaces $S=S_{s}$ for any tensorfield $F$.

$$
\|F\|_{L^{p}(S)} \lesssim\|\nabla F\|_{L^{2}(S)}^{1-\frac{2}{p}} \cdot\|F\|_{L^{2}(S)}^{\frac{2}{p}}+\|F\|_{L^{2}(S)}, \quad 2 \leq p<\infty
$$

Also, for every $2 \leq p<\infty$,

$$
\|F\|_{L^{\infty}(S)} \lesssim\left\|\nabla^{2} F\right\|_{L^{2}(S)}^{\frac{1}{p}}\|\nabla F\|_{L^{2}(S)}^{\frac{p-2}{p}}\|F\|_{L^{2}(S)}^{\frac{1}{p}}+\|\nabla F\|_{L^{2}(S)}
$$

As a consequence of the Böchner identity for tensors, see proposition 3.2

$$
\begin{aligned}
\left\|\nabla^{2} F\right\|_{L^{2}(S)} & \lesssim\|\Delta F\|_{L^{2}(S)}+\|K\|_{L^{2}(S)}^{\frac{p}{p-1}}\|\nabla F\|_{L^{2}(S)}^{\frac{p-2}{p-1}}\|F\|_{L^{2}(S)}^{\frac{1}{p-1}} \\
& +\|K\|_{L^{2}(S)}\|\nabla F\|_{L^{2}(S)}
\end{aligned}
$$


while for scalars $f$,

$$
\left\|\nabla^{2} f\right\|_{L^{2}(S)}+\|\nabla f\|_{L^{2}(S)} \lesssim\|\Delta f\|_{L^{2}(S)}
$$

Proof : For the first three inequalities see [Kl-Rodn2]. The proof of (42) can be found in [Kl-Rodn1] section 4.

3.16. Properties of the heat flow. Given a tensor $F$ on $S=S_{s}$ we define the corresponding heat flow $U(\tau) F$ to be the unique solution of the equation,

$$
\partial_{\tau} U(\tau) F-\Delta U(\tau) F=0, \quad U(0) F=F .
$$

Here $\Delta$ denotes the standard Laplace-Beltrami operator on tensors,

$$
\Delta G=\gamma^{i j} \nabla_{i} \nabla_{j} G .
$$

Proposition 3.17. The heat flow $U(\tau) F$ verifies the following properties:

$$
\begin{aligned}
\|U(\tau) F\|_{L^{p}(S)} & \lesssim\|F\|_{L^{p}(S)}, \quad 1 \leq p \leq \infty \\
\|\nabla U(\tau) F\|_{L^{2}(S)} & \lesssim\|\nabla F\|_{L^{2}(S)} \\
\|\nabla U(\tau) F\|_{L^{2}(S)} & \lesssim \tau^{-\frac{1}{2}}\|F\|_{L^{2}(S)} \\
\|U(\tau) \nabla F\|_{L^{2}(S)} & \lesssim \tau^{-\frac{1}{2}}\|F\|_{L^{2}(S)} \\
\|\Delta U(\tau) F\|_{L^{2}(S)} & \lesssim \tau^{-1}\|F\|_{L^{2}(S)}
\end{aligned}
$$

Also, for $2 \leq p<\infty$,

$$
\|U(\tau) F\|_{L^{p}(S)} \lesssim\left(1+\tau^{-(1-2 / p)}\right)\|F\|_{L^{2}(S)}
$$

and the dual estimate, for $1<q \leq 2$,

$$
\|U(\tau) F\|_{L^{2}(S)} \lesssim\left(1+\tau^{(1-2 / q)}\right)\|F\|_{L^{q}(S)}
$$

In addition, if $f$ is a scalar function ${ }^{7}$

$$
\|U(\tau) f\|_{L^{\infty}(S)} \lesssim\left(1+\tau^{-1}\right)\|f\|_{L^{2}(S)}
$$

and its dual

$$
\|U(\tau) f\|_{L^{2}(S)} \lesssim\left(1+\tau^{-1}\right)\|f\|_{L^{1}(S)}
$$

Proof : See [Kl-Rodn2].

\footnotetext{
${ }^{7}$ We do not know if such estimate holds in the tensor case. This failure is also connected with the absence of strong tensor Bernstein inequality, to be discussed in the next subsection.
} 
3.18. Geometric LP-projections. Finally we recall below the definition and main properties of the Littlwood-Paley(LP) projections introduced in [Kl-Rodn2].

Definition 3.19. Consider the class $\mathcal{M}$ of smooth functions $m$ on $[0, \infty)$, vanishing sufficiently fast at $\infty$, verifying the vanishing moments property:

$$
\int_{0}^{\infty} \tau^{k_{1}} \partial_{\tau}^{k_{2}} m(\tau) d \tau=0, \quad\left|k_{1}\right|+\left|k_{2}\right| \leq N
$$

We set,

$$
m_{k}(\tau)=2^{2 k} m\left(2^{2 k} \tau\right)
$$

and define the geometric Littlewood -Paley (LP) projections $P_{k}$, associated to the LP- representative function $m \in \mathcal{M}$, for arbitrary tensorfields $F$ on a given surface $S=S_{s}, 0 \leq s \leq 1$, to be

$$
P_{k} F=\int_{0}^{\infty} m_{k}(\tau) U(\tau) F d \tau
$$

where $U(\tau) F$ is the heat flow on $S$.

Given an interval $I \subset \mathbb{Z}$ we define

$$
P_{I}=\sum_{k \in I} P_{k} f
$$

In particular we shall use the notation $P_{<k}, P_{\leq k}, P_{>k}, P_{\geq k}$.

Observe that $P_{k}$ are selfadjoint. They verify the following properties:

Proposition 3.20. The following properties of the LP projections depend only on the conditions $\mathbf{W S}, \mathbf{K} \mathbf{1}, \mathbf{K} \mathbf{2}$.

i) $L^{p}$-boundedness For any $1 \leq p \leq \infty$, and any interval $I \subset \mathbb{Z}$,

$$
\left\|P_{I} F\right\|_{L^{p}(S)} \lesssim\|F\|_{L^{p}(S)}
$$

ii) $\quad L^{p}$ - almost orthogonality Consider two families of LP-projections $P_{k}, \tilde{P}_{k}$ associated to $m$ and respectively $\tilde{m}$, both in $\mathcal{M}$. For any $1 \leq p \leq \infty$ :

$$
\left\|P_{k} \tilde{P}_{k^{\prime}} F\right\|_{L^{p}(S)} \lesssim 2^{-2\left|k-k^{\prime}\right|}\|F\|_{L^{p}(S)}
$$

iii) Bessel inequality

$$
\sum_{k}\left\|P_{k} F\right\|_{L^{2}(S)}^{2} \lesssim\|F\|_{L^{2}(S)}^{2}
$$

iv) Reproducing property ${ }^{8} \quad$ Given an appropriately defined $\bar{m} \in \mathcal{M}$ there exists $m \in \mathcal{M}$ such that such that $\bar{m}=m \star m$. Thus,

$$
{ }^{(\bar{m})} P_{k}={ }^{(m)} P_{k} \cdot{ }^{(m)} P_{k} .
$$

Whenever there is no danger of confusion we shall simply write $P_{k}=P_{k} \cdot P_{k}$.

\footnotetext{
${ }^{8}$ see precise statement in[Kl-Rodn1].
} 
v) Finite band property For any $1 \leq p \leq \infty, k \geq 0$,

$$
\begin{array}{rlr}
\left\|\Delta P_{k} F\right\|_{L^{p}(S)} & \lesssim 2^{2 k}\|F\|_{L^{p}(S)} & {[\Delta \mathbf{F B}]} \\
\left\|P_{k} F\right\|_{L^{p}(S)} & \lesssim 2^{-2 k}\|\Delta F\|_{L^{p}} & {\left[\Delta \mathbf{F B}^{-\mathbf{1}}\right]}
\end{array}
$$

In addition, the $L^{2}$ estimates

$$
\begin{array}{rlr}
\left\|\nabla P_{k} F\right\|_{L^{2}(S)} & \lesssim 2^{k}\|F\|_{L^{2}(S)} & {[\nabla \mathbf{F B}]} \\
\left\|P_{k} F\right\|_{L^{2}(S)} & \lesssim 2^{-k}\|\nabla F\|_{L^{2}} & {\left[\nabla \mathbf{F B}^{-\mathbf{1}}\right]}
\end{array}
$$

hold together with the dual estimate

$$
\left\|P_{k} \nabla F\right\|_{L^{2}(S)} \lesssim 2^{k}\|F(S)\|_{L^{2}}
$$

vi) Weak Bernstein inequality. For any $2 \leq p<\infty$

$$
\begin{array}{ll}
\left\|P_{k} F\right\|_{L^{p}(S)} & \lesssim\left(2^{\left(1-\frac{2}{p}\right) k}+1\right)\|F\|_{L^{2}(S)}, \\
\left\|P_{<0} F\right\|_{L^{p}(S)} \lesssim\|F\|_{L^{2}(S)} & {[\mathbf{w B}]}
\end{array}
$$

together with the dual estimates

$$
\begin{array}{ll}
\left\|P_{k} F\right\|_{L^{2}(S)} \lesssim\left(2^{\left(1-\frac{2}{p}\right) k}+1\right)\|F\|_{L^{p^{\prime}}(S)}, & {\left[\mathbf{w B}^{*}\right]} \\
\left\|P_{<0} F\right\|_{L^{2}(S)} & \lesssim\|F\|_{L^{p^{\prime}}(S)}
\end{array}
$$

vii) Strong Scalar Bernstein Inequality For any scalar function $f$ and $k \geq 0$

$$
\begin{array}{ll}
\left\|P_{k} f\right\|_{L^{\infty}(S)} & \lesssim 2^{k}\|f\|_{L^{2}(S)} \\
\left\|P_{<0} f\right\|_{L^{\infty}(S)} \lesssim\|f\|_{L^{2}(S)} & {[\mathbf{s s B}],}
\end{array}
$$

and the dual estimates,

$$
\begin{aligned}
\left\|P_{k} f\right\|_{L^{1}(S)} & \lesssim 2^{k}\|f\|_{L^{2}(S)} \\
\left\|P_{<0} f\right\|_{L^{1}(S)} & \lesssim\|f\|_{L^{2}(S)}
\end{aligned}
$$

In addition we have the following curvature dependent estimates.

viii) Strong Tensor Bernstein Inequality For any tensor-field $F, k \geq 0$

$$
\begin{aligned}
& \left\|P_{k} F\right\|_{L^{\infty}(S)} \lesssim\left(2^{k}+2^{k \frac{p-2}{p-1}}\|K\|_{L^{2}(S)}^{\frac{1}{p-1}}\right) \cdot\|F\|_{L^{2}(S)}, \quad[\mathbf{s t B}] \\
& \left\|P_{<0} F\right\|_{L^{\infty}(S)} \lesssim\left(1+\|K\|_{L^{2}(S)}^{\frac{1}{p-1}}\right) \cdot\|F\|_{L^{2}(S)}
\end{aligned}
$$

ix) Dyadic Böchner inequality For any tensor-field $F$ and $2 \leq p<\infty, k \geq 0$,

$$
\begin{aligned}
& \left\|\nabla^{2} P_{k} F\right\|_{L^{2}(S)} \lesssim\left(2^{2 k}+2^{k}\|K\|_{L^{2}(S)}+2^{k \frac{p-2}{p-1}}\|K\|_{L^{2}(S)}^{\frac{p}{p-1}}\right) \cdot\|F\|_{L^{2}(S)}, \\
& \left\|\nabla^{2} P_{<0} F\right\|_{L^{2}(S)} \lesssim\left(1+\|K\|_{L^{2}(S)}+\|K\|_{L^{2}(S)}^{\frac{p}{p-1}}\right) \cdot\|F\|_{L^{2}(S)}
\end{aligned}
$$

x) Dyadic $L^{\infty}$ inequality For any tensor-field $F$ and $2 \leq p<\infty, k \geq 0$,

$$
\begin{aligned}
& \left\|P_{k} F\right\|_{L^{\infty}} \lesssim\left(2^{k}+2^{k \frac{p-1}{p}}\|K\|_{L^{2}}^{\frac{1}{p}}+2^{k \frac{p-2}{p-1}}\|K\|_{L^{2}}^{\frac{1}{p-1}}\right) \cdot\|F\|_{L^{2}}, \\
& \left\|P_{<0} F\right\|_{L^{\infty}} \lesssim\left(1+\|K\|_{L^{2}}^{\frac{1}{p}}+\|K\|_{L^{2}}^{\frac{1}{p-1}}\right) \cdot\|F\|_{L^{2}}
\end{aligned}
$$


Proof: See [Kl-Rodn2].

In what follows we outline some of the main differences between the properties of geometric LP theory projections recorded in theorem 3.20 and the properties LP1-LP5.

(1) The simple, pointwise, orthogonality property LP1 does not hold. The replacement by the almost orthogonality (55) is not going to create major difficulties, however the usual trichotomy properties of products are not longer valid. More precisely, in the classical LP theory low-low interactions of the type ${ }^{9} P_{k}\left(f_{<k} \cdot g_{<k}\right)$ are forbidden. This is no longer valid for our geometric LP theory.

(2) The geometric LP projections commute with the geometric laplacean $\Delta$ but fail to commute with covariant derivatives. Because of this one has to be very careful when applying the finite band properties recorded in $\mathrm{v}$ ).

(3) In our applications to null hypersurfaces we don't have a bound ${ }^{10}$ for the quantity $\left\|K-\frac{1}{r^{2}}\right\|_{L^{2}(S)}$. Because of this we have to be very careful when we apply the strong Bernstein estimates $\left(L^{2}-L^{\infty}\right.$ ) for tensorfields. However, we do have an unconditional strong Bernstein inequality for scalars.

(4) In flat Minkowski space both the classical and geometric LP projections commute $^{11}$ with $\nabla_{L}$ derivatives. This is no longer true for the geometric LP projections for null hypersurfaces on curved backgrounds. Moreover, due to our weak regularity assumptions BA1 - BA2 as well as WS, K1, $\mathbf{K 2}$, the commutators are often not any better, in terms of their regularity properties, than the principal term.

\subsection{Besov spaces on surfaces $S=S_{s}$.}

The following result was proved in [Kl-Rodn1]

\section{Proposition 3.22.}

i.) Consider the LP projections $P_{k}$ associated to an arbitrary $m \in \mathcal{M}_{2}$. Then,

$$
\begin{aligned}
\sum_{k}\left\|P_{k} f\right\|_{L^{2}(S)}^{2} & \lesssim\|f\|_{L^{2}(S)}^{2} \\
\sum_{k} 2^{2 k}\left\|P_{k} f\right\|_{L^{2}(S)}^{2} & \lesssim\|\nabla f\|_{L^{2}(S)}^{2}
\end{aligned}
$$

ii.) If in addition the LP-projections $P_{k}$ verify:

$$
\sum_{k} P_{k}^{2}=I
$$

\footnotetext{
${ }^{9}$ In fact $P_{k}\left(f_{<k-2} \cdot g_{<k-2}\right)$

${ }^{10}$ According to assumption $\mathbf{K} 1$ we only control $\left\|K-\frac{1}{r^{2}}\right\|_{L^{2}(\mathcal{H})}$. The only bound for the Gauss curvature, on a fixed surface $S=S_{s}$, we posses is given by $\mathbf{K 2}$.

${ }^{11}$ modulo a $\frac{1}{r}$ term generated by the mean curvature of the sphere foliation of the Minkowski null cone.
} 
Then,

$$
\begin{aligned}
\|f\|_{L^{2}(S)}^{2} & =\sum_{k}\left\|P_{k} f\right\|_{L^{2}(S)}^{2} \\
\|\nabla f\|_{L^{2}(S)}^{2} & \lesssim \sum_{k} 2^{2 k}\left\|P_{k} f\right\|_{L^{2}(S)}^{2}
\end{aligned}
$$

Using a family of LP projections $P_{k}$ verifying $\sum P_{k}^{2}=I$ we can now define our main Besov type norms:

Definition 3.23. Given an arbitrary tensor $F$ on a fixed $S=S_{s}$ we define the Besov norm $B_{2,1}^{a}(S)$ for every $0 \leq \alpha<\infty$,

$$
\|F\|_{B_{2,1}^{a}(S)}=\left(\sum_{k \geq 0} 2^{a k}\left\|P_{k} F\right\|_{L^{2}(S)}\right)+\left\|\sum_{k<0} P_{k} F\right\|_{L^{2}(S)} .
$$

We recall the following product estimates:

Proposition 3.24. Let $a, a^{\prime}, b, b^{\prime}>0$ such that $a+b=a^{\prime}+b^{\prime} \geq 1$. Then for all tensorfields $F, G$ and any $0 \leq c<1$,

$$
\|F \cdot G\|_{B_{2,1}^{c}(S)} \lesssim\left\|\Lambda^{a+c} F\right\|_{L^{2}(S)}\left\|\Lambda^{b} G\right\|_{L^{2}(S)}+\left\|\Lambda^{a^{\prime}} G\right\|_{L^{2}(S)}\left\|\Lambda^{b^{\prime}+c} G\right\|_{L^{2}(S)}
$$

Proof: See [Kl-Rodn2].

We shall also need the following estimate connecting the norms $B_{2,1}^{1}\left(S_{0}\right)$ and $B_{2,1}^{0}\left(S_{0}\right)$ for scalars.

Proposition 3.25. Given a scalar function $f$ on $S$ we have the inequality:

$$
\|f\|_{B_{2,1}^{1}(S)} \lesssim\|f\|_{B_{2,1}^{0}(S)}+\|\nabla f\|_{B_{2,1}^{0}(S)}
$$

Proof : See [Kl-Rodn2].

\subsection{Besov spaces on null hypersurfaces $\mathcal{H}$.}

Using the geometric LP projections we are ready to define our main Besov type norms on $\mathcal{H}$.

Definition 3.27. For $S$-tangent tensors $F$ on $\mathcal{H}$ we introduce the norms, for $0 \leq$ $a \leq 1$ :

$$
\begin{aligned}
\|F\|_{\mathcal{B}^{a}} & =\sum_{k \geq 0} 2^{a k}\left\|P_{k} F\right\|_{L_{t}^{\infty} L_{x}^{2}}+\left\|P_{<0} F\right\|_{L_{t}^{\infty} L_{x}^{2}}, \\
\|F\|_{\mathcal{P}^{a}} & =\sum_{k \geq 0} 2^{a k}\left\|P_{k} F\right\|_{L_{t}^{2} L_{x}^{2}}+\left\|P_{<0} F\right\|_{L_{t}^{2} L_{x}^{2}}
\end{aligned}
$$


The following is a crucial result allowing us to pass from tensorial $\mathcal{B}^{0}$ estimates to their $\mathcal{B}^{0}$ scalar counterparts.

Proposition 3.28. There exist a finite number of vectorfields $X_{1}, \ldots X_{l}$ verifying the following properties ${ }^{12}$,

$$
\|X, \stackrel{\circ}{\nabla} X\|_{L_{t}^{\infty} L_{x}^{\infty}} \lesssim 1, \quad\|(\nabla-\stackrel{\circ}{\nabla}) X\|_{L_{x}^{2} L_{t}^{\infty}} \lesssim \Delta_{0}, \quad\|\nabla(\stackrel{\circ}{\nabla} X)\|_{L_{x}^{2} L_{t}^{\infty}} \lesssim 1
$$

An arbitrary $S$-tangent tensor $F \in L_{t}^{\infty} L_{x}^{2}$ is in $\mathcal{B}^{0}$ if and only if $F \cdot X_{i} \in \mathcal{B}^{0}$ for all $1 \leq i \leq l$. Moreover the $X_{i}$ 's can be chosen to be coordinate vectorfields with $\nabla_{L} X_{i}=0$.

Proof : To prove the proposition we first choose the vectorfields $X$ to be the coordinate vectorfields associated to the transported coordinates $(t, \omega)$. The conditions $\|X\|_{L_{t}^{\infty} L_{x}^{\infty}} \lesssim 1$ and $\|(\nabla-\stackrel{\circ}{\nabla}) X\|_{L_{x}^{2} L_{t}^{\infty}} \lesssim \Delta_{0}$ are equivalent to our WS condition. Moreover $\stackrel{\circ}{\nabla}^{2} X$ are clearly bounded and the condition on $\nabla \stackrel{\circ}{\nabla} X$ follows from $\|\partial \gamma\|_{L_{X}^{2} L_{t}^{\infty}} \lesssim 1$.

With the help of these vectorfields we note the following characterization of the $L_{t}^{\infty} L_{x}^{2}$ norm for tensors ${ }^{13} F$.

$$
\|F\|_{L_{t}^{\infty} L_{x}^{2}} \approx \max _{1 \leq i \leq l}\left\|X_{i} \cdot F\right\|_{L_{t}^{\infty} L_{x}^{2}}
$$

We now proceed as follows ${ }^{14}$ :

$$
\begin{aligned}
\|F\|_{\mathcal{B}^{0}} & =\sum_{k \geq 0}\left\|P_{k} F\right\|_{L_{t}^{\infty} L_{x}^{2}} \approx \max _{1 \leq i \leq l} \sum_{k \geq 0}\left\|X_{i} \cdot P_{k} F\right\|_{L_{t}^{\infty} L_{x}^{2}} \\
& =\max _{1 \leq i \leq l} \sum_{k \geq 0}\left\|P_{k}\left(X_{i} \cdot F\right)\right\|_{L_{t}^{\infty} L_{x}^{2}}+O\left(\sum_{k \geq 0}\left\|\left[P_{k}, X\right] \cdot F\right\|_{L_{t}^{\infty} L_{x}^{2}}\right)
\end{aligned}
$$

It suffices to prove that,

$$
\begin{aligned}
\sum_{k \geq 0}\left\|\left[P_{k}, X\right] \cdot F\right\|_{L_{t}^{\infty} L_{x}^{2}} & \lesssim\|(\nabla-\stackrel{\circ}{\nabla}) X\|_{L_{x}^{2} L_{t}^{\infty}}\|F\|_{\mathcal{B}^{0}}+\|F\|_{L_{t}^{\infty} L_{x}^{2}} \\
& \lesssim \Delta_{0}\|F\|_{\mathcal{B}^{0}}+\|F\|_{L_{t}^{\infty} L_{x}^{2}} .
\end{aligned}
$$

\footnotetext{
12 recall that $\stackrel{\circ}{\nabla}$ represents the covariant derivative with respect to the background metric $(1+s)^{2} \stackrel{\circ}{\gamma}$

13 for simplicity of notations we restrict to 1 -forms.

${ }^{14}$ We only show the $X_{i} \cdot F \in \mathcal{B}^{0}$ implies $F \in \mathcal{B}^{0}$. This is the implication which will be needed later. Observe that we also drop the term $\left\|P_{<0} F\right\|_{L_{t}^{\infty} L_{x}^{2}} \lesssim\|F\|_{L_{t}^{\infty} L_{x}^{2}}$, which is trivial, in the expression for $\|F\|_{\mathcal{B}^{0}}$
} 
To prove (67) we have to recall the formula

$$
\begin{aligned}
{\left[P_{k}, X\right] \cdot F } & =\int_{0}^{\infty} m_{k}(\tau) \Phi(\tau) \\
\Phi(\tau ; k) & =\int_{0}^{\tau} U\left(\tau-\tau^{\prime}\right)[\Delta, X] U\left(\tau^{\prime}\right) F d \tau^{\prime}=\Phi_{1}(\tau)+\Phi_{2}(\tau)+\Phi_{3}(\tau)+\Phi_{4}(\tau) \\
\Phi_{1}(\tau ; k) & =\int_{0}^{\tau} U\left(\tau-\tau^{\prime}\right)(\nabla-\stackrel{\circ}{\nabla}) X \cdot \nabla U\left(\tau^{\prime}\right) F d \tau^{\prime} \\
\Phi_{2}(\tau ; k) & \left.=\int_{0}^{\tau} U\left(\tau-\tau^{\prime}\right) \operatorname{div}(\nabla-\stackrel{\circ}{\nabla}) X \cdot U\left(\tau^{\prime}\right) F\right) d \tau^{\prime} \\
\Phi_{3}(\tau ; k) & =\int_{0}^{\tau} U\left(\tau-\tau^{\prime}\right) \stackrel{\circ}{\nabla} X \cdot \nabla U\left(\tau^{\prime}\right) F d \tau^{\prime} \\
\Phi_{4}(\tau ; k) & =\int_{0}^{\tau} U\left(\tau-\tau^{\prime}\right) \operatorname{div}\left(\stackrel{\circ}{\nabla} X \cdot U\left(\tau^{\prime}\right) F\right) d \tau^{\prime}
\end{aligned}
$$

since

$$
\begin{aligned}
{[\Delta, X] F } & =\nabla^{i}\left(\nabla_{i} X \cdot F\right)+\nabla_{i} X \cdot \nabla^{i} F=\nabla^{i}\left(\left(\nabla_{i}-\stackrel{\circ}{\nabla}_{i}\right) X \cdot F\right)+\left(\nabla_{i}-\stackrel{\circ}{\nabla}_{i}\right) X \cdot \nabla^{i} F \\
& +\nabla^{i}\left(\stackrel{\circ}{\nabla}_{i} X \cdot F\right)+\stackrel{\circ}{\nabla}_{i} X \cdot \nabla^{i} F
\end{aligned}
$$

To estimate $\Phi_{1}$ we observe that $U\left(\tau-\tau^{\prime}\right)$ corresponds to a scalar heat flow and therefore we make use of the following scalar heat flow estimate (51):

$$
\left\|U\left(\tau-\tau^{\prime}\right) f\right\|_{L_{x}^{2}} \lesssim\left(1+\left|\tau-\tau^{\prime}\right|^{-\frac{1}{2}}\right)\|f\|_{L_{x}^{1}}
$$

Remark 3.29. In what follows, we shall systematically replace the above estimate, and all other heat flow estimates like it, with their slightly incorrect versions where we ignore the non-singular term 1 . We thus write

$$
\left\|U\left(\tau-\tau^{\prime}\right) f\right\|_{L_{x}^{2}} \lesssim\left|\tau-\tau^{\prime}\right|^{-\frac{1}{2}}\|f\|_{L_{x}^{1}}
$$

Therefore,

$$
\begin{aligned}
\left\|\Phi_{1}(\tau ; k)\right\|_{L_{t}^{\infty} L_{x}^{2}} & \lesssim \int_{0}^{\tau}\left|\tau-\tau^{\prime}\right|^{-\frac{1}{2}}\left\|(\nabla-\stackrel{\circ}{\nabla}) X \cdot \nabla U\left(\tau^{\prime}\right) F\right\|_{L_{t}^{\infty} L_{x}^{1}} \\
& \lesssim \int_{0}^{\tau}\left|\tau-\tau^{\prime}\right|^{-\frac{1}{2}}\|(\nabla-\stackrel{\circ}{\nabla}) X\|_{L_{x}^{2} L_{t}^{\infty}} \cdot\left\|\nabla U\left(\tau^{\prime}\right) F\right\|_{L_{t}^{\infty} L_{x}^{2}} \\
& \lesssim \Delta_{0} \cdot \int_{0}^{\tau}\left|\tau-\tau^{\prime}\right|^{-\frac{1}{2}}\left\|\nabla U\left(\tau^{\prime}\right) F\right\|_{L_{t}^{\infty} L_{x}^{2}}
\end{aligned}
$$

To estimate the integral

$$
J(\tau)=\int_{0}^{\tau}\left|\tau-\tau^{\prime}\right|^{-\frac{1}{2}}\left\|\nabla U\left(\tau^{\prime}\right) F\right\|_{L_{t}^{\infty} L_{x}^{2}}
$$

we decompose it as follows:

$$
J(\tau) \lesssim \sum_{m} \int_{0}^{\tau}\left|\tau-\tau^{\prime}\right|^{-\frac{1}{2}}\left\|\nabla P_{m} U\left(\tau^{\prime}\right) F\right\|_{L_{t}^{\infty} L_{x}^{2}}=\sum_{m} J_{m}(\tau)
$$

Next we make use of

Lemma 3.30.

$$
J_{m}(\tau) \lesssim \min \left(2^{m} \tau^{\frac{1}{2}}, 2^{-m} \tau^{-\frac{1}{2}}\right)\left\|P_{m} F\right\|_{L_{t}^{\infty} L_{x}^{2}}
$$


and proceed as follows:

$$
\int_{0}^{\infty} m_{k}(\tau)\left\|\Phi_{1}(\tau ; k)\right\|_{L_{t}^{\infty} L_{x}^{2}} \lesssim \sum_{m}\left\|P_{m} F\right\|_{L_{t}^{\infty} L_{x}^{2}} \int_{0}^{\infty} m_{k}(\tau) \min \left(2^{m} \tau^{\frac{1}{2}}, 2^{-m} \tau^{-\frac{1}{2}}\right)
$$

Now,

$$
\begin{aligned}
\int_{0}^{\infty} m_{k}(\tau) \min \left(2^{m} \tau^{\frac{1}{2}}, 2^{-m} \tau^{-\frac{1}{2}}\right) & =2^{m} \int_{0}^{2^{-2 m}} m_{k}(\tau) \tau^{\frac{1}{2}}+2^{-m} \int_{2^{-2 m}}^{\infty} m_{k}(\tau) \tau^{-\frac{1}{2}} \\
& =2^{m-k} \int_{0}^{2^{2(k-m)}} \tilde{m}(\tau)+2^{k-m} \int_{2^{2(k-m)}}^{\infty} \hat{m}(\tau) \\
& \lesssim 2^{-|k-m|}
\end{aligned}
$$

Here $\tilde{m}(\tau)=\tau^{\frac{1}{2}} m(\tau)$ and $\hat{m}(\tau)=\tau^{-\frac{1}{2}} m(\tau)$. Moreover, to arrive at the inequality above we used the following bounds:

$$
\int_{0}^{\infty} \tilde{m}(\tau), \quad \int_{0}^{\infty} \hat{m}(\tau) \lesssim 1, \quad \int_{0}^{a} \tilde{m}(\tau) \lesssim a, \quad \int_{A}^{\infty} \hat{m}(\tau) \lesssim A^{-1}
$$

which hold for all sufficiently small $a$ and all sufficiently large $A$. Thus,

$$
\sum_{k \geq 0} \int_{0}^{\infty} m_{k}(\tau)\left\|\Phi_{1}(\tau ; k)\right\|_{L_{t}^{2} L_{x}^{2}} \lesssim \Delta_{0} \sum_{k \geq 0} \sum_{m} 2^{-|m-k|}\left\|P_{m} F\right\|_{L_{t}^{2} L_{x}^{2}} \lesssim \Delta_{0}\|F\|_{\mathcal{P}^{0}}
$$

To estimate $\Phi_{2}$ we first observe that the following estimate for the scalar heat flow holds for any $1<p \leq 2$ :

$$
\left\|U\left(\tau-\tau^{\prime}\right) \operatorname{div} g\right\|_{L_{x}^{2}} \lesssim\left(\tau-\tau^{\prime}\right)^{-\frac{1}{p}}\|g\|_{L_{x}^{p}}
$$

Using this we obtain for some $p<2$ sufficiently close to $p=2$,

$$
\begin{aligned}
\left\|\Phi_{2}(\tau ; k)\right\|_{L_{t}^{\infty} L_{x}^{2}} & \lesssim \int_{0}^{\tau}\left(\tau-\tau^{\prime}\right)^{-\frac{1}{p}}\left\|(\nabla-\stackrel{\circ}{\nabla}) X \cdot U\left(\tau^{\prime}\right) F\right\|_{L_{t}^{\infty} L_{x}^{p}} \\
& \lesssim \int_{0}^{\tau}\left(\tau-\tau^{\prime}\right)^{-\frac{1}{p}}\|(\nabla-\stackrel{\circ}{\nabla}) X\|_{L_{x}^{2} L_{t}^{\infty}}\left\|U\left(\tau^{\prime}\right) F\right\|_{L_{t}^{\infty} L_{x}^{\frac{2 p}{2-p}}} \\
& \lesssim \Delta_{0} \sum_{m} \int_{0}^{\tau}\left(\tau-\tau^{\prime}\right)^{-\frac{1}{p}}\left\|P_{m} U\left(\tau^{\prime}\right) F\right\|_{L_{t}^{\infty} L_{x}^{\frac{2 p}{2-p}}} \\
& \lesssim \Delta_{0} \sum_{m} \int_{0}^{\tau}\left(\tau-\tau^{\prime}\right)^{-\frac{1}{p}}\left\|\nabla P_{m} U\left(\tau^{\prime}\right) F\right\|_{L_{t}^{\infty} L_{x}^{2}}^{2-\frac{2}{p}}\left\|P_{m} U\left(\tau^{\prime}\right) F\right\|_{L_{t}^{\infty} L_{x}^{2}}^{\frac{2}{p}-1}
\end{aligned}
$$

The remaining argument now is a straightforward modification of the proof for $\Phi_{1}$. We infer that

$\sum_{k \geq 0} \int_{0}^{\infty} m_{k}(\tau)\left\|\Phi_{2}(\tau ; k)\right\|_{L_{t}^{\infty} L_{x}^{2}} \lesssim \Delta_{0} \sum_{k \geq 0} \sum_{m} 2^{-\left(2-\frac{2}{p}\right)|k-m|}\left\|P_{m} F\right\|_{L_{t}^{\infty} L_{x}^{2}} \lesssim \Delta_{0}\|F\|_{\mathcal{B}^{0}}$

It only remains to estimate the easier terms $\Phi_{3}, \Phi_{4}$.

$$
\begin{aligned}
\Phi_{3}(\tau ; k) & =\int_{0}^{\tau} U\left(\tau-\tau^{\prime}\right) \stackrel{\circ}{\nabla} X \cdot \nabla U\left(\tau^{\prime}\right) F d \tau^{\prime}=\Phi_{31}(\tau ; k)+\Phi_{32}(\tau ; k) \\
& =\int_{0}^{\tau} U\left(\tau-\tau^{\prime}\right)(\nabla \stackrel{\circ}{\nabla} X) \cdot U\left(\tau^{\prime}\right) F d \tau^{\prime}+\int_{0}^{\tau} U\left(\tau-\tau^{\prime}\right) \nabla\left(\stackrel{\circ}{\nabla} X \cdot U\left(\tau^{\prime}\right) F\right) d \tau^{\prime}
\end{aligned}
$$


Now, starting as for $\Phi_{1}, \Phi_{2}$,

$$
\begin{aligned}
\left\|\Phi_{31}(\tau ; k)\right\|_{L_{t}^{\infty} L_{x}^{2}} & \lesssim \int_{0}^{\tau}\left(\tau-\tau^{\prime}\right)^{-\frac{1}{2}}\left\|\nabla \stackrel{\circ}{\nabla} X \cdot U\left(\tau^{\prime}\right) F\right\|_{L_{t}^{\infty} L_{x}^{1}} \\
& \lesssim \int_{0}^{\tau}\left(\tau-\tau^{\prime}\right)^{-\frac{1}{2}}\|\nabla \stackrel{\circ}{\nabla} X\|_{L_{x}^{2} L_{t}^{\infty}} \cdot\left\|U\left(\tau^{\prime}\right) F\right\|_{L_{t}^{\infty} L_{x}^{2}} \lesssim \tau^{\frac{1}{2}}\|F\|_{L_{t}^{\infty} L_{x}^{2}} \\
\left\|\Phi_{32}(\tau ; k)\right\|_{L_{t}^{\infty} L_{x}^{2}} & \lesssim \int_{0}^{\tau}\left(\tau-\tau^{\prime}\right)^{-\frac{1}{2}}\left\|\stackrel{\circ}{\nabla} X \cdot U\left(\tau^{\prime}\right) F\right\|_{L_{t}^{\infty} L_{x}^{2}} \lesssim \tau^{\frac{1}{2}}\|F\|_{L_{t}^{\infty} L_{x}^{2}}
\end{aligned}
$$

Hence,

$$
\sum_{k \geq 0} \int_{0}^{\infty} m_{k}(\tau)\left\|\Phi_{3}(\tau ; k)\right\|_{L_{t}^{\infty} L_{x}^{2}} \lesssim \sum_{k \geq 0} \int_{0}^{\infty} \tau^{\frac{1}{2}} m_{k}(\tau) d \tau \lesssim \sum_{k} 2^{-k}
$$

and similarly for $\Phi_{4}$. Thus, going back to (69) we have

$$
\sum_{k}\left\|\left[P_{k}, X\right] F\right\|_{L_{t}^{\infty} L_{x}^{2}} \lesssim \Delta_{0}\|F\|_{\mathcal{B}^{0}}+\|F\|_{L_{t}^{\infty} L_{x}^{2}}
$$

Proof of lemma 3.30. Recall that our goal is to prove the estimate

$$
\int_{0}^{\tau}\left(\tau-\tau^{\prime}\right)^{-\frac{1}{2}}\left\|\nabla P_{m} U\left(\tau^{\prime}\right) F\right\|_{L_{t}^{\infty} L_{x}^{2}} \lesssim \min \left(2^{m} \tau^{\frac{1}{2}}, 2^{-m} \tau^{-\frac{1}{2}}\right)\left\|P_{m} F\right\|_{L_{t}^{\infty} L_{x}^{2}} .
$$

We first observe that,

$$
\left\|\nabla P_{m} U\left(\tau^{\prime}\right) F\right\|_{L_{t}^{\infty} L_{x}^{2}} \lesssim 2^{m} \min \left(1,2^{-4 m}\left(\tau^{\prime}\right)^{-2}\right)\left\|P_{m} F\right\|_{L_{t}^{\infty} L_{x}^{2}}
$$

Indeed we have both

$$
\left\|\nabla P_{m} U\left(\tau^{\prime}\right) F\right\|_{L_{t}^{\infty} L_{x}^{2}} \lesssim 2^{m}\left\|P_{m} F\right\|_{L_{t}^{\infty} L_{x}^{2}}
$$

and

$$
\begin{aligned}
\left\|\nabla P_{m} U\left(\tau^{\prime}\right) F\right\|_{L_{t}^{\infty} L_{x}^{2}} & \lesssim 2^{m}\left\|P_{m} U\left(\tau^{\prime}\right) F\right\|_{L_{t}^{\infty} L_{x}^{2}} \\
& \lesssim 2^{m} 2^{-4 m}\left\|P_{m} \Delta^{2} U\left(\tau^{\prime}\right) F\right\|_{L_{t}^{\infty} L_{x}^{2}} \\
& \lesssim 2^{m} 2^{-4 m}\left(\tau^{\prime}\right)^{-2}\left\|P_{m} F\right\|_{L_{t}^{\infty} L_{x}^{2}}
\end{aligned}
$$

To show that,

$$
\begin{aligned}
I_{m}(\tau) & =\left\|P_{m} F\right\|_{L_{t}^{\infty} L_{x}^{2}}^{-1} \cdot \int_{0}^{\tau}\left(\tau-\tau^{\prime}\right)^{-\frac{1}{2}}\left\|\nabla P_{m} U\left(\tau^{\prime}\right) F\right\|_{L_{t}^{\infty} L_{x}^{2}} \\
& \lesssim \min \left(2^{m} \tau^{\frac{1}{2}}, 2^{-m} \tau^{-\frac{1}{2}}\right)
\end{aligned}
$$

it suffices to prove

$$
\begin{aligned}
& I_{m}(\tau) \lesssim 2^{m} \tau^{\frac{1}{2}} \quad \text { if } \quad \tau \leq 2^{-2 m} \\
& I_{m}(\tau) \lesssim 2^{-m} \tau^{-\frac{1}{2}} \quad \text { if } \quad \tau \geq 2^{-2 m}
\end{aligned}
$$

Using (71) we infer that,

$$
I_{m}(\tau) \lesssim 2^{m} \int_{0}^{\tau}\left(\tau-\tau^{\prime}\right)^{-\frac{1}{2}} \min \left(1,2^{-4 m}\left(\tau^{\prime}\right)^{-2}\right) d \tau^{\prime}
$$


For $\tau \leq 2^{-2 m}$ we have,

$$
I_{m}(\tau) \lesssim 2^{m} \int_{0}^{\tau}\left(\tau-\tau^{\prime}\right)^{-\frac{1}{2}} d \tau^{\prime} \lesssim 2^{m} \tau^{\frac{1}{2}}
$$

For $\tau \geq 2^{-2 m}$,

$$
\begin{aligned}
I_{m}(\tau) & \lesssim 2^{-3 m} \int_{2^{-2 m}}^{\tau}\left(\tau-\tau^{\prime}\right)^{-\frac{1}{2}} \cdot\left(\tau^{\prime}\right)^{-2} d \tau^{\prime}+2^{m} \int_{0}^{2^{-2 m}}\left(\tau-\tau^{\prime}\right)^{-\frac{1}{2}} d \tau^{\prime} \\
& \lesssim 2^{-m} \tau^{-\frac{1}{2}}
\end{aligned}
$$

as desired.

\section{MAIn RESUlts}

We are now ready to state our main results. They can be viewed as extensions of results mentioned in the introduction, see proposition 1.5, to null hypersurfaces $\mathcal{H}$ verifying the assumptions A1, A2, WS and K1, K2. The first is a generalized sharp bilinear trace theorem.

Theorem 4.1 (Bilinear-Trace-Transport). Consider the transport equation along $\mathcal{H}$ :

$$
\nabla_{L} W=\nabla_{L} F \cdot G
$$

for $S$-tangent tensors $W, F, G$. Then,

$$
\|W\|_{\mathcal{B}^{0}} \lesssim\left\|\left.W\right|_{S_{0}}\right\|_{B_{2,1}^{0}\left(S_{0}\right)}+\mathcal{N}_{1}[F] \cdot\left(\mathcal{N}_{1}[G]+\|G\|_{L_{x}^{\infty} L_{t}^{2}}\right)
$$

Remark 4.2. We have a stronger estimate in the case of a transport equation for a scalar function $w$.

$$
\|w\|_{\mathcal{B}^{0}} \lesssim\left\|\left.w\right|_{S_{0}}\right\|_{B_{2,1}^{0}\left(S_{0}\right)}+\mathcal{N}_{1}[F] \cdot \mathcal{N}_{1}[G]
$$

The next two theorems are the noncommutative versions of the sharp product estimates of proposition 1.3.

Theorem 4.3 (Product-Transport I). Consider the transport equation along $\mathcal{H}$ :

$$
\nabla_{L} W=F \cdot G
$$

for $S$-tangent tensors $W, F, G$. We have the estimate,

$$
\|W\|_{\mathcal{B}^{0}} \lesssim\left\|\left.W\right|_{S_{0}}\right\|_{B_{2,1}^{0}\left(S_{0}\right)}+\|F\|_{\mathcal{P}^{0}} \cdot\left(\mathcal{N}_{1}[G]+\|G\|_{L_{x}^{\infty} L_{t}^{2}}\right)
$$

Theorem 4.4 (Product-Transport II). Given any pair of $S$-tangent tensors $G, W$, of same type such that $W$ satisfies a transport equation, of the form,

$$
\nabla_{L} W=F
$$

Then,

$$
\|G \cdot W\|_{\mathcal{P}^{0}} \lesssim\left(\|F\|_{\mathcal{P}^{0}}+\left\|\left.W\right|_{S_{0}}\right\|_{B_{2,1}^{0}\left(S_{0}\right)}\right) \cdot\left(\mathcal{N}_{1}[G]+\|G\|_{L_{x}^{\infty} L_{t}^{2}}\right)
$$

As a consequence of theorems 4.1, 4.3 we derive the following. 
Theorem 4.5 (Sharp-Trace). For any $S$-tangent tensor $F$, which allows a decomposition of the form $\nabla F=\nabla_{L} \check{F}+G$, we have

$$
\|F\|_{L_{x}^{\infty} L_{t}^{2}} \lesssim \mathcal{N}_{1}[F]+\mathcal{N}_{1}[\check{F}]+\|G\|_{\mathcal{P}^{0}}
$$

Proof : The proof can be found in section 5 of [Kl-Rodn1]. The idea is to introduce the scalar function $f(t)=\int_{0}^{t}|F|^{2}$ and observe that it verifies the transport equation,

Differentiating it one derives,

$$
\nabla_{L} f=|F|^{2}, \quad U(0)=0 .
$$

$$
\begin{aligned}
\nabla_{L}(\nabla f)+\frac{1}{2} \operatorname{tr} \chi(\nabla f) & =2 F \cdot \nabla F-\hat{\chi} \cdot(\nabla f) \\
& =2 F \cdot \nabla_{L} \check{F}+2 F \cdot G-\hat{\chi} \cdot(\nabla f)
\end{aligned}
$$

and apply ${ }^{15}$ propositions 4.1, 4.3 .

In particular, we have the following noncommutative version of the classical sharp trace theorem.

Corollary 4.6. For any $S$-tangent tensor $F$

$$
\int_{0}^{t}\left|\nabla_{L} F\right|^{2} \lesssim \int_{\mathcal{H}}\left(\left|\nabla^{2} F\right|^{2}+\left|\nabla_{L}^{2} F\right|^{2}+|F|^{2}\right)
$$

\subsection{Reduction to scalar estimates.}

The first two transport theorems can be reduced to the case of a scalar transport equation. More precisely, we have the following

Proposition 4.8. Assume that the conclusions of theorems 4.1 and 4.3 have been verified for scalar transport equations. Then they also hold true in the tensor case. Moreover, in the particular case of theorems 4.3, and 4.4 we can reduce the corresponding estimates to a fully scalar situation, i.e both $F$ and $G$ are scalar functions.

Proof : In view of the scalar characterization of the space $\mathcal{B}^{0}$ stated in proposition 3.28 it suffices to do the following. We multiply the transport equation for a tensor ${ }^{16}$ $W$, in either of the theorems, by the vectorfields $X$ to derive a scalar equation

$$
\nabla_{L}(X \cdot W)=\nabla_{L} F \cdot(G \widehat{\otimes} X) \quad(\text { or } F \cdot(G \widehat{\otimes} X))
$$

where $\widehat{\otimes}$ denotes either a tensor product or a contraction. It only remains to observe that the norm $\mathcal{N}_{1}[G \widehat{\otimes} X]+\|G \widehat{\otimes} X\|_{L_{x}^{\infty} L_{t}^{2}}$ is invariant with respect to a tensor multiplication by a vectorfield $X$ with the properties guaranteed by proposition 3.28. To prove the second part of the proposition consider the case of theorem 4.3 where we have already reduced to the case $\nabla_{L} w=F \cdot G$ with $w$ scalar and $F \cdot G$ denotes the scalar product between two tensorfields. Clearly $F \cdot G$ can be expressed, at every point, as a product between various scalar components of $F$ and $G$ inner

\footnotetext{
${ }^{15}$ One has to take some care to eliminate the term $\operatorname{tr} \chi \cdot \nabla f$ first, as it is done in [Kl-Rodn1].

${ }^{16}$ Assume for simplicity that $W$ is a 1 -form
} 
product with the vectorfields $X_{i}$ and the components of the metric $\gamma$. Therefore we can apply the proposition 3.28 to each of the scalar components ${ }^{17}$ and derive our result in view of the invariance of the norms involved.

The reduction to scalar equations is a very important simplification in so far as it allows us to work with integral estimates. We state below a result which, in view of the reduction made above, implies theorems 4.1, 4.3.

Theorem 4.9. The following statements hold true for arbitrary $S$ tangent tensorfields $F, G$ of same order ${ }^{18}$ :

$$
\begin{gathered}
\left\|\int_{0}^{t} \nabla_{L} F \cdot G\right\|_{\mathcal{B}^{0}} \lesssim \mathcal{N}_{1}[F] \cdot \mathcal{N}_{1}[G] \\
\left\|\int_{0}^{t} F \cdot G\right\|_{\mathcal{B}^{0}} \lesssim\|F\|_{\mathcal{P}^{0}} \cdot\left(\mathcal{N}_{1}[G]+\|G\|_{L_{x}^{\infty} L_{t}^{2}}\right)
\end{gathered}
$$

Moreover for solutions of the homogeneous scalar transport equation $\nabla_{L} w=0$ we have,

$$
\|w\|_{\mathcal{B}^{0}} \lesssim\left\|\left.w\right|_{S_{0}}\right\|_{\mathcal{B}^{0}}
$$

Indeed, once we are in the scalar case, for example in the case of theorem 4.1, $\nabla_{L} w=F \cdot G$, we can integrate and therefore reduce the statement of the theorem to,

$$
\sum_{k}\left\|P_{k} \int_{0}^{t} F \cdot G\right\|_{L_{t}^{\infty} L_{x}^{2}} \lesssim \mathcal{N}_{1}[F] \cdot\left(\mathcal{N}_{1}[G]+\|G\|_{L_{x}^{\infty} L_{t}^{2}}\right)
$$

Remark 4.10. We can also prove a more precise dyadic version of the estimate (79): for any $k \geq 0$ and some $\sigma>0$,

$$
\begin{aligned}
\left\|P_{k} \int_{0}^{t} F \cdot G\right\|_{L_{t}^{\infty} L_{x}^{2}} \lesssim & \left(\sum_{k^{\prime}} 2^{-\sigma\left|k-k^{\prime}\right|}\left\|P_{k^{\prime}} F\right\|_{L_{t}^{2} L_{x}^{2}}+2^{-\sigma k}\|F\|_{L_{t}^{2} L_{x}^{2}}\right) \\
& \left(\mathcal{N}_{1}[G]+\|G\|\right)
\end{aligned}
$$

\section{Some DyAdic ESTimates}

In the proof of the theorems we shall need the notion of an $\mathcal{N}_{1}$ envelope of a tensorfield. It plays the role of an LP -localized version of the $\mathcal{N}_{1}$ norm of definition 3.7 .

\footnotetext{
${ }^{17}$ The components of the metric $\gamma$ can be combined with $G$.

18 such that $F \cdot G$ denotes a scalar.
} 
Definition 5.1. For a given smooth $S$-tangent tensor-field $F$ and a sufficiently small $\epsilon>0$ we define its $\mathcal{N}_{1}$-envelope (of $\operatorname{order}^{19} \epsilon$ ) to be any sequence of positive real numbers $\mathcal{N}_{1}\left[F_{k}\right]$ satisfying the following properties:

$$
\begin{aligned}
\mathcal{N}_{1}\left[F_{k}\right] & \lesssim 2^{\epsilon\left|k-k^{\prime}\right|} \mathcal{N}_{1}\left[F_{k^{\prime}}\right], \quad \text { for any } k, k^{\prime}, \\
\sum_{k} \mathcal{N}_{1}\left[F_{k}\right]^{2} & \approx \mathcal{N}_{1}[F]^{2}
\end{aligned}
$$

The existence of an envelope follows from the following elementary construction. Let $\overline{\mathcal{N}_{1}}\left[F_{k}\right]$, be defined as follows:

$$
\overline{\mathcal{N}}_{1}\left[F_{k}\right]=\left\|F_{k}\right\|_{L_{t}^{2} L_{x}^{2}}+\left\|\nabla F_{k}\right\|_{L_{t}^{2} L_{x}^{2}}+\left\|\left(\nabla_{L} F\right)_{k}\right\|_{L_{t}^{2} L_{x}^{2}},
$$

Note that, in view of proposition 3.22,

$$
\sum_{k} \bar{N}_{1}\left[F_{k}\right]^{2} \approx \mathcal{N}_{1}[F]^{2}
$$

We now easily check that the sequences

$$
\mathcal{N}_{1}\left[F_{k}\right]=\sum_{k^{\prime}} 2^{-\epsilon\left|k-k^{\prime}\right|} \overline{\mathcal{N}}_{1}\left[F_{k^{\prime}}\right]
$$

are desired envelopes. The following simple result provides us with a useful tool in handling various error terms.

Lemma 5.2. Let $\left\{\mathcal{N}_{1}\left[F_{k}\right]\right\}$ be an envelope (of order $\epsilon$ ) for a tensor-field $F$. Then for any $0<\alpha \leq \epsilon$ the sequence $\left\{\mathcal{N}_{1}\left[F_{k}\right]+2^{-\alpha k} \mathcal{N}_{1}[F]\right\}$ is also an envelope. Moreover, for any $\alpha>\epsilon$ the sequence $\left\{\mathcal{N}_{1}\left[F_{k}\right]+2^{-\alpha k} \mathcal{N}_{1}[F]\right\}$ is dominated by an $\mathcal{N}_{1}$-envelope for $F$.

Proof : The first part of the lemma is obvious from the definition. From a purely technical point of view $\left\{\mathcal{N}_{1}\left[F_{k}\right]+2^{-\alpha k} \mathcal{N}_{1}[F]\right\}$ is not an an envelope of order $\epsilon$ if $\alpha \geq \epsilon$ yet it can be clearly dominated by one.

We now formulate and prove a number of results which will be routinely used in the proof of our main theorem. The next result allows us to treat the multitude of commutator terms with $\nabla_{L}$ which will appear throughout the next sections.

Lemma 5.3. For any smooth $S$-tangent tensor field $F$ and all $q<2$ sufficiently close to $q=2^{20}$,

$$
\left\|\left[P_{k}, \nabla_{L}\right] F\right\|_{L_{t}^{q} L_{x}^{2}}+2^{-k}\left\|\nabla\left[P_{k}, \nabla_{L}\right] F\right\|_{L_{t}^{q} L_{x}^{2}} \lesssim 2^{-\frac{k}{2}+} \mathcal{N}_{1}[F]
$$

while for $q=1$,

$$
\left\|\left[P_{k}, \nabla_{L}\right] F\right\|_{L_{t}^{1} L_{x}^{2}}+2^{-k}\left\|\nabla\left[P_{k}, \nabla_{L}\right] F\right\|_{L_{t}^{1} L_{x}^{2}} \lesssim 2^{-k+} \mathcal{N}_{1}[F]
$$

Proof : See section 12.

As a corollary of this we see how to control the $\nabla_{L}$-derivative of the LP pieces of a tensor-field $f$ in terms of its envelope.

\footnotetext{
${ }^{19}$ Unless otherwise specified we shall assume that $\epsilon$ is a fixed, sufficiently small constant.

${ }^{20} \mathrm{By} 2^{-\frac{k}{2}+}$ we mean $2^{-a k}$ with $a<1 / 2$ arbitrarily close to $1 / 2$.
} 
Lemma 5.4. For all $q, 1 \leq q<2$ sufficiently close to $q=2$ and any smooth $S$-tangent tensor-field $F$,

$$
\left\|\nabla_{L} F_{k}\right\|_{L_{t}^{q} L_{x}^{2}} \lesssim \mathcal{N}_{1}\left[F_{k}\right]
$$

for some $\mathcal{N}_{1}$-envelope for $f$.

Proof : We write

$$
\nabla_{L} F_{k}=P_{k} \nabla_{L} F+\left[P_{k}, \nabla_{L}\right] F
$$

The commutator estimate of Lemma 5.3 implies that

$$
\left\|\left[P_{k}, \nabla_{L}\right] F\right\|_{L_{t}^{q} L_{x}^{2}} \lesssim 2^{-\frac{k}{2}} \mathcal{N}_{1}[F]
$$

Thus,

$$
\left\|\nabla_{L} F_{k}\right\|_{L_{t}^{q} L_{x}^{2}} \lesssim\left\|P_{k} \nabla_{L} F\right\|_{L_{t}^{q} L_{x}^{2}}+2^{-\frac{k}{2}} \mathcal{N}_{1}[F] \lesssim \mathcal{N}_{1}\left[F_{k}\right]
$$

The above result can be complemented by a dyadic Gagliardo-Nirenberg estimate with respect to the time variable $t$.

Lemma 5.5. For any smooth $S$-tangent tensor field $f$, any $2 \leq q \leq \infty$, we have the following dyadic Gagliardo-Nirenberg inequality

$$
\left\|F_{k}\right\|_{L_{t}^{q} L_{x}^{2}} \lesssim 2^{-k\left(\frac{1}{2}+\frac{1}{q}\right)} \mathcal{N}_{1}\left[F_{k}\right] \quad \quad\left[\mathbf{G N}_{\mathbf{k}}\right]
$$

Proof : First, we trivially estimate

$$
\left\|F_{k}\right\|_{L_{t}^{q} L_{x}^{2}}^{q} \lesssim\left\|F_{k}\right\|_{L_{t}^{2} L_{x}^{2}}^{2}\left\|F_{k}\right\|_{L_{x}^{2} L_{t}^{\infty}}^{q-2}
$$

On the other hand,

$$
\begin{aligned}
\left\|F_{k}\right\|_{L_{t}^{\infty}}^{2} & \lesssim\left\|\nabla_{L} F_{k} \cdot f_{k}\right\|_{L_{t}^{1}}+\left\|F_{k}\right\|_{L_{t}^{2}}^{2} \\
& \lesssim\left\|\left(\nabla_{L} F\right)_{k} \cdot F_{k}\right\|_{L_{t}^{1}}+\left\|\left[P_{k}, \nabla_{L}\right] F \cdot F_{k}\right\|_{L_{t}^{1}}+\left\|F_{k}\right\|_{L_{t}^{2}}^{2}
\end{aligned}
$$

Thus,

$$
\left\|F_{k}\right\|_{L_{x}^{2} L_{t}^{\infty}}^{2} \lesssim\left\|\left(\nabla_{L} F\right)_{k}\right\|_{L_{t}^{2} L_{x}^{2}}\left\|F_{k}\right\|_{L_{t}^{2} L_{x}^{2}}+\left\|\left[P_{k}, \nabla_{L}\right] F \cdot F_{k}\right\|_{L_{t}^{1} L_{x}^{1}}+\left\|F_{k}\right\|_{L_{t}^{2} L_{x}^{2}}^{2}
$$

We choose an exponent $r<2$ sufficiently close to $r=2$ and estimate the commutator term as follows.

$$
\begin{aligned}
\left\|\left[P_{k}, \nabla_{L}\right] F \cdot F_{k}\right\|_{L_{t}^{1} L_{x}^{1}} & \lesssim\left\|\left[P_{k}, \nabla_{L}\right] F\right\|_{L_{t}^{r} L_{x}^{2}}\left\|F_{k}\right\|_{L_{t}^{r^{\prime}} L_{x}^{2}} \\
& \lesssim 2^{-\frac{k}{2}} \mathcal{N}_{1}[F]\left\|F_{k}\right\|_{L_{t}^{r^{\prime}} L_{x}^{2}}
\end{aligned}
$$

Combining all the estimates we obtain that

$$
\left\|F_{k}\right\|_{L_{t}^{q} L_{x}^{2}}^{q} \lesssim\left\|F_{k}\right\|_{L_{t}^{2} L_{x}^{2}}^{2}\left(\left\|\left(\nabla_{L} F\right)_{k}\right\|_{L_{t}^{2} L_{x}^{2}}\left\|F_{k}\right\|_{L_{t}^{2} L_{x}^{2}}+2^{-\frac{k}{2}} \mathcal{N}_{1}[F]\left\|F_{k}\right\|_{L_{t}^{r^{\prime}} L_{x}^{2}}+\left\|F_{k}\right\|_{L_{t}^{2} L_{x}^{2}}\right)^{\frac{q}{2}-1}
$$

It follows that for all $r^{\prime}>2$ sufficiently close to $r^{\prime}=2$,

$$
\left\|F_{k}\right\|_{L_{t}^{q} L_{x}^{2}} \lesssim\left\|\left(\nabla_{L} F\right)_{k}\right\|_{L_{t}^{2} L_{x}^{2}}^{\frac{1}{2}-\frac{1}{q}}\left\|F_{k}\right\|_{L_{t}^{2} L_{x}^{2}}^{\frac{1}{2}+\frac{1}{2}}+\left\|F_{k}\right\|_{L_{t}^{2} L_{x}^{2}}+2^{-\frac{k}{2}\left(\frac{1}{2}-\frac{1}{q}\right)}\left(\mathcal{N}_{1}[F]\right)^{\frac{1}{2}-\frac{1}{q}}\left\|F_{k}\right\|_{L_{t}^{\prime \prime} L_{x}^{2}}^{\frac{1}{2}-\frac{1}{q}}\left\|F_{k}\right\|_{L_{t}^{2} L_{x}^{2}}^{\frac{2}{q}}
$$


Using the above estimate with $q=r^{\prime}$ and then plugging the result into the above estimate for a given $q$, we obtain that for any $\alpha<\frac{1}{4}-\frac{1}{2 q}$,

$$
\left\|F_{k}\right\|_{L_{t}^{q} L_{x}^{2}} \lesssim 2^{-k\left(\frac{1}{2}+\frac{1}{q}\right)}\left(\mathcal{N}_{1}\left[F_{k}\right]+2^{-\alpha k} \mathcal{N}_{1}[F]\right) .
$$

The desired result now easily follows.

Lemma 5.6. For any smooth $S$-tangent tensor-field $F$ and any Lebesque exponent $2 \leq q<4$,

$$
\left\|\nabla F_{k}\right\|_{L_{t}^{q} L_{x}^{4}} \lesssim 2^{k\left(1-\frac{1}{q}\right)} \mathcal{N}_{1}\left[F_{k}\right]
$$

Proof By Gagliardo-Nirenberg (39),

$$
\left\|\nabla F_{k}\right\|_{L_{t}^{q} L_{x}^{4}} \lesssim\|\| \nabla^{2} F_{k}\left\|_{L_{x}^{2}}^{\frac{1}{2}}\right\| \nabla F_{k}\left\|_{L_{x}^{2}}^{\frac{1}{2}}\right\|_{L_{t}^{q}}
$$

On the other hand, according to the Bochner inequality ${ }^{21}$ (41),

$$
\left\|\nabla^{2} F_{k}\right\|_{L_{x}^{2}} \lesssim\left\|\Delta F_{k}\right\|_{L_{x}^{2}}+\|K\|_{L_{x}^{2}}^{\frac{p}{p-1}}\left\|\nabla F_{k}\right\|_{L_{x}^{2}}
$$

for any $2 \leq p<\infty$. Choose the exponent $p$ such that

$$
2=\frac{q p}{2(p-1)}
$$

The existence of such $p$ is guaranteed by the condition that $q<4$. Thus, using Hölder inequality and condition K1,

$$
\begin{aligned}
\left\|\nabla F_{k}\right\|_{L_{t}^{q} L_{x}^{4}} & \lesssim\left\|\Delta F_{k}\right\|_{L_{t}^{q} L_{x}^{2}}^{\frac{1}{2}}\left\|\nabla F_{k}\right\|_{L_{t}^{q} L_{x}^{2}}^{\frac{1}{2}}+\left\|\nabla F_{k}\right\|_{L_{t}^{\infty} L_{x}^{2}} \\
& \lesssim 2^{\frac{3 k}{2}}\left\|F_{k}\right\|_{L_{t}^{q} L_{x}^{2}}+2^{k}\left\|F_{k}\right\|_{L_{t}^{\infty} L_{x}^{2}}
\end{aligned}
$$

It remains to apply the Gagliardo-Nirenberg inequality $\left[\mathbf{G N}_{\mathbf{k}}\right]$ of Lemma 5.5.

Our next result is the integrated version of the strong Bernstein inequality.

Lemma 5.7. For any $S$-tangent tensor-field $F$ and exponent $2 \leq q<\infty$,

$$
\left\|F_{k}\right\|_{L_{t}^{q} L_{x}^{\infty}} \lesssim 2^{k\left(\frac{1}{2}-\frac{1}{q}\right)} \mathcal{N}_{1}\left[F_{k}\right]
$$

Proof : Observe that the dyadic $L^{\infty}$ inequality of x) of proposition 3.20 implies that for all sufficiently large $p$

$$
\left\|F_{k}\right\|_{L_{x}^{\infty}} \lesssim 2^{k}\left(1+2^{-\frac{1}{p} k}\|K\|_{L_{x}^{2}}^{\frac{1}{p}}+2^{-\frac{1}{p-1} k}\|K\|_{L_{x}^{2}}^{\frac{1}{p-1}}\right)\left\|F_{k}\right\|_{L_{x}^{2}}
$$

Taking the $L_{t}^{q}$ norm we and using the condition $\mathbf{K} 1$ we obtain

$$
\left\|F_{k}\right\|_{L_{x}^{\infty}} \lesssim 2^{k}\left\|F_{k}\right\|_{L_{t}^{q} L_{x}^{2}}+2^{-\frac{1}{p} k}\left\|F_{k}\right\|_{L_{t}^{\frac{2 q p}{2 p-q}} L_{x}^{2}}+2^{-\frac{1}{p-1} k}\left\|F_{k}\right\|_{L_{t}^{\frac{2 q(p-1)}{2(p-1)-q}} L_{x}^{2}}
$$

Thus, applying the dyadic Gagliardo-Nirenberg estimate of Lemma 5.5 we derive

$$
\begin{aligned}
\left\|F_{k}\right\|_{L_{x}^{\infty}} & \lesssim\left(2^{k\left(\frac{1}{2}-\frac{1}{q}\right)}+2^{-\frac{1}{p} k} \cdot 2^{k\left(\frac{1}{2}-\frac{2 p-q}{2 p q}\right)}+2^{-\frac{1}{p-1} k} \cdot 2^{k\left(\frac{1}{2}-\frac{2(p-1)-q}{2(p-1) q}\right)}\right) \mathcal{N}_{1}\left[F_{k}\right] \\
& \lesssim 2^{k\left(\frac{1}{2}-\frac{1}{q}\right)} \cdot\left(1+2^{-\frac{1}{2 p} k}+2^{-\frac{1}{2(p-1)} k}\right) \mathcal{N}_{1}\left[F_{k}\right] \\
& \lesssim 2^{k\left(\frac{1}{2}-\frac{1}{q}\right)} \mathcal{N}_{1}\left[F_{k}\right]
\end{aligned}
$$

\footnotetext{
${ }^{21}$ Simplified a bit and ignoring lower order terms.
} 
as desired.

\section{Notations. Outline of the Remainder of the PAPER}

In the proof we shall often refer to various properties of the LP calculus and other analytical tools developed above. To help the reader we give below a glossary of our main notations:

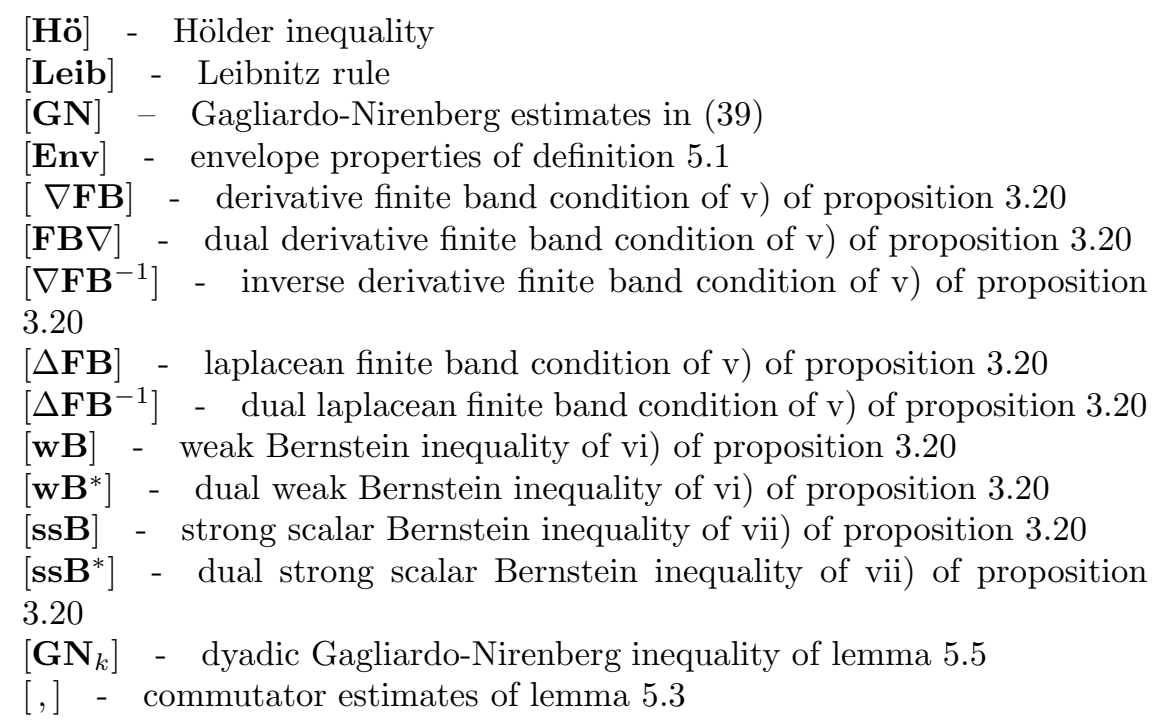

The remainder of the paper is focused on the proof of theorems 4.4 and 4.9 .

We start with section 7 where we prove an integration by parts lemma which is the noncommutative counterpart of lemma 2.2 .

Section 8 contains the proof of the sharp bilinear trace estimate of theorem 4.9. The proof follows the outline of the corresponding flat statement with modifications taking into the account non-commutativity and absence of the strong tensor Bernstein inequality.

Sections 9 and 10 provide the proofs of the integrated sharp product estimates I and II of theorems 4.9 and 4.4 .

In section 11 we finish the proof of theorem 4.9 by establishing the $\mathcal{B}^{0}$ estimates for solutions of a homogeneous scalar transport equation. The proof is quickly reduced to an estimate for the commutator $\left[\nabla_{L}, P_{k}\right]$ applied to a scalar $\mathcal{B}^{0}$ function.

Finally, section 12 contains the proof of non-sharp tensor commutator estimates for $\left[\nabla_{L}, P_{k}\right]$ needed to control various error terms throughout the paper. 


\section{DyADIC INTEGRATION BY PARTS}

In this section we shall deal with an integration by parts lemma which is needed in the proof of the sharp bilinear trace theorem of theorem 4.9. The lemma is the non-commutative analogue of the integration by parts argument in the proof of the flat sharp bilinear trace estimate of proposition 1.2.

We shall estimate the time integral of the expression

$$
" \nabla_{L} "\left(F_{k^{\prime}} \cdot G_{k^{\prime \prime}}\right):=\left(\nabla_{L} F\right)_{k^{\prime}} \cdot G_{k^{\prime \prime}}+F_{k^{\prime}} \cdot\left(\nabla_{L} G\right)_{k^{\prime \prime}}
$$

where $F, G$ are tensors of the same order and - denotes the scalar product. Observe that the expression above differs from the perfect derivative $\frac{d}{d s}\left(F_{k^{\prime}} \cdot G_{k^{\prime \prime}}\right)$ by the commutator terms

$$
\left[P_{k^{\prime}}, \nabla_{L}\right] F \cdot G_{k^{\prime \prime}}, \quad F_{k^{\prime}} \cdot\left[P_{k^{\prime \prime}}, \nabla_{L}\right] G
$$

Proposition 7.1. Let $F, G$ be tensors of same order. Then for any $k, k^{\prime}, k^{\prime \prime}$,

$$
\left\|P_{k} \int_{0}^{t} " \nabla_{L} "\left(F_{k^{\prime}} \cdot G_{k^{\prime \prime}}\right)\right\|_{L_{t}^{\infty} L_{x}^{2}} \lesssim 2^{-\sigma\left(\left|k^{\prime}-k^{\prime \prime}\right|+\left|k^{\prime}-k\right|\right)} \mathcal{N}_{1}\left[F_{k^{\prime}}\right] \cdot \mathcal{N}_{1}\left[G_{k^{\prime \prime}}\right]
$$

with a strictly positive $\sigma$ independent of $k, k^{\prime}, k^{\prime \prime}$.

Proof : By symmetry it suffices to consider the following three cases:
a) $k^{\prime} \geq k^{\prime \prime} \geq k$
b) $k^{\prime} \geq k>k^{\prime \prime}$,
c) $k>k^{\prime} \geq k^{\prime \prime}$

In all of the cases the proof of the proposition reduces to the estimate for the commutators

$$
\begin{aligned}
&\left\|P_{k} \int_{0}^{t}\left[P_{k^{\prime}}, \nabla_{L}\right] F \cdot G_{k^{\prime \prime}}\right\|_{L_{t}^{\infty} L_{x}^{2}} \lesssim 2^{-\sigma\left(\left|k^{\prime}-k^{\prime \prime}\right|+\left|k^{\prime}-k\right|\right)} \mathcal{N}_{1}\left[F_{k^{\prime}}\right] \cdot \mathcal{N}_{1}\left[G_{k^{\prime \prime}}\right] \\
&\left\|P_{k} \int_{0}^{t} F_{k^{\prime}} \cdot\left[P_{k^{\prime \prime}}, \nabla_{L}\right] G\right\|_{L_{t}^{\infty} L_{x}^{2}} \lesssim 2^{-\sigma\left(\left|k^{\prime}-k^{\prime \prime}\right|+\left|k^{\prime}-k\right|\right)} \mathcal{N}_{1}\left[F_{k^{\prime}}\right] \cdot \mathcal{N}_{1}\left[G_{k^{\prime \prime}}\right]
\end{aligned}
$$

as well as the estimate for the boundary terms

$$
\left\|P_{k}\left(F_{k^{\prime}}(t) \cdot G_{k^{\prime \prime}}(t)-F_{k^{\prime}}(0) \cdot G_{k^{\prime \prime}}(0)\right)\right\|_{L_{t}^{\infty} L_{x}^{2}} \lesssim 2^{-\sigma\left(\left|k^{\prime}-k^{\prime \prime}\right|+\left|k^{\prime}-k\right|\right)} \mathcal{N}_{1}\left[F_{k^{\prime}}\right] \cdot \mathcal{N}_{1}\left[G_{k^{\prime \prime}}\right]
$$

Since $k^{\prime \prime} \leq k^{\prime}$ the estimate (89) is more sensitive than (88) and we shall only prove it and (90) in what follows.

We start with the easier of the three case.

a) We start by applying the dual strong Bernstein inequality for scalars followed by Cauchy-Schwartz with $q<2$, the dyadic Gagliardo-Nirenberg inequality of Lemma 
5.5 and the commutator estimate of Lemma 5.3, to infer that

$$
\begin{aligned}
\left\|P_{k} \int_{0}^{t} F_{k^{\prime}} \cdot\left[P_{k^{\prime \prime}}, \nabla_{L}\right] G\right\|_{L_{t}^{\infty} L_{x}^{2}} & \lesssim 2^{k}\left\|F_{k^{\prime}} \cdot\left[P_{k^{\prime \prime}}, \nabla_{L}\right] G\right\|_{L_{t}^{1} L_{x}^{1}} \\
& \lesssim 2^{k}\left\|F_{k^{\prime}}\right\|_{L_{t}^{q^{\prime}} L_{x}^{2}}\left\|\left[P_{k^{\prime \prime}}, \nabla_{L}\right] G\right\|_{L_{t}^{q} L_{x}^{2}} \quad[\mathbf{H o ̈}] \\
{\left[\mathbf{G N}_{k}\right] \&[,] } & \lesssim 2^{k} 2^{-k^{\prime}\left(\frac{1}{2}+\frac{1}{q^{\prime}}\right)} 2^{-\frac{k^{\prime \prime}}{2}} \mathcal{N}_{1}\left[F_{k^{\prime}}\right] \cdot \mathcal{N}_{1}[G] \\
& \lesssim 2^{-\left(\frac{1}{2}+\frac{1}{q^{\prime}}\right)\left(k^{\prime}-k^{\prime \prime}\right)} 2^{-\left(k^{\prime \prime}-k\right)} \mathcal{N}_{1}\left[F_{k^{\prime}}\right] \cdot 2^{-\frac{1}{q^{\prime}} k^{\prime \prime}} \mathcal{N}_{1}[G] \\
{[\text { Env }] } & \lesssim 2^{-\left(\frac{1}{2}+\frac{1}{q^{\prime}}\right)\left(k^{\prime}-k^{\prime \prime}\right)} 2^{-\left(k^{\prime \prime}-k\right)} \mathcal{N}_{1}\left[F_{k^{\prime}}\right] \cdot \mathcal{N}_{1}\left[G_{k^{\prime \prime}}\right]
\end{aligned}
$$

The estimate for the boundary terms proceeds as follows. Using the dual strong Bernstein inequality for scalars followed by the Cauchy-Schwartz and the dyadic Gagliardo-Nirenberg estimate of Lemma 5.5 with $q=\infty$, we obtain

$$
\begin{array}{rlrl}
\left\|P_{k}\left(F_{k^{\prime}} \cdot G_{k^{\prime \prime}}\right)\right\|_{L_{t}^{\infty} L_{x}^{2}} & \lesssim 2^{k}\left\|F_{k^{\prime}} \cdot G_{k^{\prime \prime}}\right\|_{L_{t}^{\infty} L_{x}^{1}} & & {\left[\mathbf{s s B}^{*}\right]} \\
& \lesssim 2^{k}\left\|F_{k^{\prime}}\right\|_{L_{t}^{\infty} L_{x}^{2}}\left\|G_{k^{\prime \prime}}\right\|_{L_{t}^{\infty} L_{x}^{2}} & & {[\mathbf{H o ̈}]} \\
& \lesssim 2^{k-\frac{k^{\prime}}{2}-\frac{k^{\prime \prime}}{2}} \mathcal{N}_{1}\left[F_{k^{\prime}}\right] \cdot \mathcal{N}_{1}\left[G_{k^{\prime \prime}}\right] & & {\left[\mathbf{G N}_{k}\right]} \\
& \lesssim 2^{-\frac{1}{2}\left(k^{\prime}-k^{\prime \prime}\right)} 2^{-\left(k^{\prime \prime}-k\right)} \mathcal{N}_{1}\left[F_{k^{\prime}}\right] \cdot \mathcal{N}_{1}\left[G_{k^{\prime \prime}}\right] &
\end{array}
$$

b) We apply the Hölder inequality with $q<2$ followed by the Gagliardo-Nirenebrg and weak Bernstein inequalities in the $x$ variable, the dyadic Gagliardo-Nirenberg inequality of Lemma 5.5, and finally the commutator estimates of Lemma 5.3, to infer that

$$
\begin{aligned}
\left\|P_{k} \int_{0}^{t} F_{k^{\prime}} \cdot\left[P_{k^{\prime \prime}}, \nabla_{L}\right] G\right\|_{L_{t}^{\infty} L_{x}^{2}} & \lesssim\left\|F_{k^{\prime}}\right\|_{L_{t}^{q^{\prime}} L_{x}^{4}}\left\|\left[P_{k^{\prime \prime}}, \nabla_{L}\right] G\right\|_{L_{t}^{q} L_{x}^{4}} \quad[\mathbf{H o ̈}] \\
{[\mathbf{w B}] \&[\mathbf{G N}] } & \lesssim 2^{\frac{k^{\prime}}{2}}\left\|F_{k^{\prime}}\right\|_{L_{t}^{q^{\prime}} L_{x}^{2}}\left\|\nabla\left[P_{k^{\prime \prime}}, \nabla_{L}\right] G\right\|_{L_{t}^{q} L_{x}^{2}}^{\frac{1}{2}}\left\|\left[P_{k^{\prime \prime}}, \nabla_{L}\right] G\right\|_{L_{t}^{q} L_{x}^{2}}^{\frac{1}{2}} \\
{\left[\mathbf{G N}_{k}\right] \&[,] } & \lesssim 2^{-\frac{1}{q^{\prime}} k^{\prime}} \mathcal{N}_{1}\left[F_{k^{\prime}}\right] \cdot \mathcal{N}_{1}[G] \\
& \lesssim 2^{-\frac{1}{2 q^{\prime}}\left(k^{\prime}-k^{\prime \prime}\right)} 2^{-\frac{1}{2 q^{\prime}}\left(k-k^{\prime \prime}\right)} \mathcal{N}_{1}\left[F_{k^{\prime}}\right] \cdot 2^{-\frac{1}{q^{\prime}}} \mathcal{N}^{\prime \prime}[G] \\
{[\mathbf{E n v}] } & \lesssim 2^{-\frac{1}{2 q^{\prime}}\left(k^{\prime}-k^{\prime \prime}\right)} 2^{-\frac{1}{2 q^{\prime}}\left(k-k^{\prime \prime}\right)} \mathcal{N}_{1}\left[F_{k^{\prime}}\right] \cdot \mathcal{N}_{1}\left[G_{k^{\prime \prime}}\right]
\end{aligned}
$$

The boundary terms are estimated with the help of the Hölder inequality followed by the weak Bernstein inequality and the dyadic Gagliardo-Nirenberg estimate of Lemma 5.5.

$$
\begin{aligned}
\left\|P_{k}\left(F_{k^{\prime}} \cdot G_{k^{\prime \prime}}\right)\right\|_{L_{t}^{\infty} L_{x}^{2}} & \lesssim\left\|F_{k^{\prime}}\right\|_{L_{t}^{\infty} L_{x}^{\frac{8}{3}}}\left\|G_{k^{\prime \prime}}\right\|_{L_{t}^{\infty} L_{x}^{8}} \\
{[\mathbf{w B}] } & \lesssim 2^{\frac{1}{4} k^{\prime}+\frac{3}{4} k^{\prime \prime}}\left\|F_{k^{\prime}}\right\|_{L_{t}^{\infty} L_{x}^{2}}\left\|G_{k^{\prime \prime}}\right\|_{L_{t}^{\infty} L_{x}^{2}} \\
{\left[\mathbf{G N}_{k}\right] } & \lesssim 2^{-\frac{1}{4}\left(k^{\prime}-k^{\prime \prime}\right)} \mathcal{N}_{1}\left[F_{k^{\prime}}\right] \cdot \mathcal{N}_{1}\left[G_{k^{\prime \prime}}\right] \\
& \lesssim 2^{-\frac{1}{8}\left(k^{\prime}-k^{\prime \prime}\right)} 2^{-\frac{1}{8}\left(k-k^{\prime \prime}\right)} \mathcal{N}_{1}\left[F_{k^{\prime}}\right] \cdot \mathcal{N}_{1}\left[G_{k^{\prime \prime}}\right]
\end{aligned}
$$

c) We start by applying the inverse finite band condition followed by Lemma 3.11 and Hölder inequality with an exponent $1 \leq q<2$ chosen to be sufficiently close to 


$$
\begin{aligned}
q=2, & \\
\left\|P_{k} \int_{0}^{t} F_{k^{\prime}} \cdot\left[P_{k^{\prime \prime}}, \nabla_{L}\right] G\right\|_{L_{t}^{\infty} L_{x}^{2}} & \lesssim 2^{-k}\left\|\nabla \int_{0}^{t} F_{k^{\prime}} \cdot\left[P_{k^{\prime \prime}}, \nabla_{L}\right] G\right\|_{L_{t}^{\infty} L_{x}^{2}} \quad\left[\nabla \mathbf{F B}^{-\mathbf{1}}\right] \\
& \lesssim 2^{-k}\left\|\nabla\left(F_{k^{\prime}} \cdot\left[P_{k^{\prime \prime}}, \nabla_{L}\right] G\right)\right\|_{L_{t}^{1} L_{x}^{2}} \\
& \lesssim 2^{-k}\left\|\nabla F_{k^{\prime}}\right\|_{L_{t}^{q^{\prime}} L_{x}^{4}}\left\|\left[P_{k^{\prime \prime}}, \nabla_{L}\right] G\right\|_{L_{t}^{q} L_{x}^{4}} \\
& +2^{-k}\left\|F_{k^{\prime}}\right\|_{L_{t}^{q^{\prime}} L_{x}^{\infty}}\left\|\nabla\left[P_{k^{\prime \prime}}, \nabla_{L}\right] G\right\|_{L_{t}^{q} L_{x}^{2}} \\
& =C_{k k^{\prime} k^{\prime \prime}}^{(1)}+C_{k k^{\prime} k^{\prime \prime}}^{(2)}
\end{aligned}
$$

To estimate the first term above we use the derivative Bernstein inequality of Lemma 5.6 together with the Gagliardo-Nirenberg estimate followed by the commutator estimates of Lemma 5.3,

$$
\begin{aligned}
C_{k k^{\prime} k^{\prime \prime}}^{(1)} & \lesssim 2^{-k} 2^{k^{\prime}\left(\frac{1}{2}-\frac{1}{q^{\prime}}\right)} \mathcal{N}_{1}\left[F_{k^{\prime}}\right] \cdot\left\|\nabla\left[P_{k^{\prime \prime}}, \nabla_{L}\right] G\right\|_{L_{t}^{q} L_{x}^{2}}^{\frac{1}{2}}\left\|\left[P_{k^{\prime \prime}}, \nabla_{L}\right] G\right\|_{L_{t}^{q} L_{x}^{2}}^{\frac{1}{2}} \\
& \lesssim 2^{-k} 2^{k^{\prime}\left(1-\frac{1}{q^{\prime}}\right.} \mathcal{N}_{1}\left[F_{k^{\prime}}\right] \cdot \mathcal{N}_{1}[G] \\
& \lesssim 2^{-\frac{1}{2 q^{\prime}}\left(k^{\prime}-k^{\prime \prime}\right)} 2^{-\frac{1}{2 q^{\prime}}\left(k-k^{\prime \prime}\right)} \mathcal{N}_{1}\left[F_{k^{\prime}}\right] \cdot 2^{-\frac{1}{q^{\prime}} k^{\prime \prime}} \mathcal{N}_{1}[G] \\
& \lesssim 2^{-\frac{1}{2 q^{\prime}}\left(k^{\prime}-k^{\prime \prime}\right)} 2^{-\frac{1}{2 q^{\prime}}\left(k-k^{\prime \prime}\right)} \mathcal{N}_{1}\left[F_{k^{\prime}}\right] \cdot \mathcal{N}_{1}\left[G_{k^{\prime \prime}}\right]
\end{aligned}
$$

To estimate the term $C_{k k^{\prime} k^{\prime \prime}}^{(2)}$ we apply the integrated version of the strong Bernstein inequality of Lemma 5.7 together with the commutator estimate of Lemma 5.3.

$$
\begin{aligned}
C_{k k^{\prime} k^{\prime \prime}}^{(2)} & \lesssim 2^{-k} 2^{k^{\prime}\left(\frac{1}{2}-\frac{1}{q^{\prime}}\right)} 2^{-\frac{k^{\prime \prime}}{2}} \mathcal{N}_{1}\left[F_{k^{\prime}}\right] \cdot \mathcal{N}_{1}[G] \\
& \lesssim 2^{-\frac{1}{q^{\prime}}\left(k^{\prime}-k^{\prime \prime}\right)} 2^{-\frac{1}{4}\left(k-k^{\prime \prime}\right)} \mathcal{N}_{1}\left[F_{k^{\prime}}\right] \cdot 2^{-k^{\prime \prime}\left(\frac{3}{4}+\frac{1}{q^{\prime}}\right)} \mathcal{N}_{1}[G] \\
& \lesssim 2^{-\frac{1}{q^{\prime}}\left(k^{\prime}-k^{\prime \prime}\right)} 2^{-\frac{1}{4}\left(k-k^{\prime \prime}\right)} \mathcal{N}_{1}\left[F_{k^{\prime}}\right] \cdot \mathcal{N}_{1}\left[G_{k^{\prime \prime}}\right]
\end{aligned}
$$

To estimate the boundary terms for the low-low interaction $\left(k^{\prime \prime} \leq k^{\prime}<k\right)$ we argue as follows. We start by applying the finite band property,

$$
\begin{aligned}
\left\|P_{k}\left(F_{k^{\prime}} \cdot G_{k^{\prime \prime}}\right)\right\|_{L_{t}^{\infty} L_{x}^{2}} & \lesssim 2^{-2 k}\left\|\Delta\left(F_{k^{\prime}} \cdot G_{k^{\prime \prime}}\right)\right\|_{L_{t}^{\infty} L_{x}^{2}} \\
{[\text { Leib] }} & \lesssim 2^{-2 k}\left\|\Delta F_{k^{\prime}} \cdot G_{k^{\prime \prime}}\right\|_{L_{t}^{\infty} L_{x}^{2}}+2^{-2 k}\left\|F_{k^{\prime}} \cdot \Delta G_{k^{\prime \prime}}\right\|_{L_{t}^{\infty} L_{x}^{2}} \\
& +2^{-2 k}\left\|P_{k}\left(\nabla F_{k^{\prime}} \cdot \nabla G_{k^{\prime \prime}}\right)\right\|_{L_{t}^{\infty} L_{x}^{2}} \\
{[\Delta \mathbf{F B}] } & \lesssim 2^{-2\left(k-k^{\prime}\right)}\left\|F_{k^{\prime}} \cdot G_{k^{\prime \prime}}\right\|_{L_{t}^{\infty} L_{x}^{2}}+2^{-2\left(k-k^{\prime \prime}\right)}\left\|F_{k^{\prime}} \cdot G_{k^{\prime \prime}}\right\|_{L_{t}^{\infty} L_{x}^{2}} \\
& +2^{-2 k}\left\|P_{k}\left(\nabla F_{k^{\prime}} \cdot \nabla G_{k^{\prime \prime}}\right)\right\|_{L_{t}^{\infty} L_{x}^{2}}
\end{aligned}
$$

The most difficult is the last term. We apply the dual strong scalar Bernstein inequality followed by Hölder inequality, finite band property, and the dyadic Gagliardo-Nirenberg inequality of Lemma 5.5,

$$
\begin{aligned}
2^{-2 k}\left\|P_{k}\left(\nabla F_{k^{\prime}} \cdot \nabla G_{k^{\prime \prime}}\right)\right\|_{L_{t}^{\infty} L_{x}^{2}} & \lesssim 2^{-k}\left\|\nabla F_{k^{\prime}} \cdot \nabla G_{k^{\prime \prime}}\right\|_{L_{t}^{\infty} L_{x}^{1}} \quad\left[\mathbf{s s B}^{*}\right] \\
{[\mathbf{H o ̈}] } & \lesssim 2^{-k}\left\|\nabla F_{k^{\prime}}\right\|_{L_{t}^{\infty} L_{x}^{2}}\left\|\nabla G_{k^{\prime \prime}}\right\|_{L_{t}^{\infty} L_{x}^{2}} \\
{[\nabla \mathbf{F B}] } & \lesssim 2^{-k+k^{\prime}+k^{\prime \prime}}\left\|F_{k^{\prime}}\right\|_{L_{t}^{\infty} L_{x}^{2}}\left\|G_{k^{\prime \prime}}\right\|_{L_{t}^{\infty} L_{x}^{2}} \\
{\left[\mathbf{G N}_{k}\right] } & \lesssim 2^{-k+\frac{k^{\prime}}{2}+\frac{k^{\prime \prime}}{2}} \mathcal{N}_{1}\left[F_{k^{\prime}}\right] \cdot \mathcal{N}_{1}\left[G_{k^{\prime \prime}}\right] \\
& \lesssim 2^{-\frac{1}{4}\left(k^{\prime}-k^{\prime \prime}\right)} 2^{-\frac{1}{4}\left(k-k^{\prime \prime}\right)} \mathcal{N}_{1}\left[F_{k^{\prime}}\right] \cdot \mathcal{N}_{1}\left[G_{k^{\prime \prime}}\right]
\end{aligned}
$$




\section{PROOF OF THE SHARP BILINEAR TRACE THEOREM}

In this section we provide the proof of the sharp bilinear trace estimate (78) of theorem 4.9:

$$
\left\|\int_{0}^{t} \nabla_{L} F \cdot G\right\|_{\mathcal{B}^{0}} \lesssim \mathcal{N}_{1}[F] \cdot \mathcal{N}_{1}[G]
$$

In fact it suffices to prove,

$$
\sum_{k \geq 0}\left\|P_{k} \int_{0}^{t} \nabla_{L} F \cdot G\right\|_{L_{t}^{\infty} L_{x}^{2}} \lesssim \mathcal{N}_{1}[F] \cdot \mathcal{N}_{1}[G] .
$$

Indeed for the low frequencies we can use the dual strong scalar Bernstein inequality $\left[\mathbf{s s B}^{*}\right]$,

$$
\begin{aligned}
\left\|P_{<0} \int_{0}^{t} \nabla_{L} F \cdot G\right\|_{L_{t}^{\infty} L_{x}^{2}} & \lesssim\left\|\int_{0}^{t} \nabla_{L} F \cdot G\right\|_{L_{t}^{\infty} L_{x}^{1}} \quad\left[\mathbf{s s B}^{*}\right] \\
& \lesssim\left\|\nabla_{L} F\right\|_{L_{t}^{2} L_{x}^{2}} \cdot\|G\|_{L_{t}^{2} L_{x}^{2}} \lesssim \mathcal{N}_{1}[F] \cdot \mathcal{N}_{1}[G] .
\end{aligned}
$$

We start with the LP - decompositions of $\nabla_{L} F \cdot G$.

$$
\begin{aligned}
\left(\nabla_{L} F \cdot G\right) & =\left(\nabla_{L} F\right)_{<k} \cdot G_{\geq k}+\left(\nabla_{L} F\right)_{\geq k} \cdot G_{<k}+\left(\nabla_{L} F\right)_{\geq k} \cdot G_{\geq k} \\
& +\left(\nabla_{L} F\right)_{<k} \cdot G_{<k}
\end{aligned}
$$

Thus,

$$
\begin{array}{cc}
P_{k} \int_{0}^{t} \nabla_{L} F \cdot G=A_{k}+B_{k}+C_{k}+D_{k} \\
A_{k}=P_{k} \int_{0}^{t}\left(\nabla_{L} F\right)_{<k} \cdot G_{\geq k}, & B_{k}=P_{k} \int_{0}^{t}\left(\nabla_{L} F\right)_{\geq k} \cdot G_{<k} \\
C_{k}=P_{k} \int_{0}^{t}\left(\nabla_{L} F\right)_{<k} \cdot G_{<k}, & D_{k}=P_{k} \int_{0}^{t}\left(\nabla_{L} F\right)_{\geq k} \cdot G_{\geq k}
\end{array}
$$

8.1. Estimates for $A_{k}=P_{k} \int_{0}^{t}\left(\nabla_{L} F\right)_{<k} \cdot G_{\geq k}$.

This is the easiest term. We start by giving a "slightly wrong" proof based on the use of strong, tensorial form, of the Bernstein inequality "[stB]":

$$
\left\|P_{k} F\right\|_{L_{x}^{\infty}} \lesssim 2^{k}\|F\|_{L_{x}^{2}}
$$

which we don't in fact possess. We shall indicate however how to circumvent this problem in remark 8.2. 
Indeed, using (94),

$$
\begin{aligned}
& \left\|A_{k}\right\|_{L_{t}^{\infty} L_{x}^{2}} \lesssim \sum_{k^{\prime}<k \leq k^{\prime \prime}} \int_{0}^{1}\left\|\left(\nabla_{L} F\right)_{k^{\prime}} \cdot G_{k^{\prime \prime}}\right\|_{L_{x}^{2}} d t \\
& \text { [Hö] } \\
& \lesssim \sum_{k^{\prime}<k \leq k^{\prime \prime}} \int_{0}^{1}\left\|\left(\nabla_{L} F\right)_{k^{\prime}}\right\|_{L_{x}^{\infty}}\left\|G_{k^{\prime \prime}}\right\|_{L_{x}^{2}} d t \\
& \text { "[stB" }] " \quad \lesssim \sum_{k^{\prime}<k \leq k^{\prime \prime}} 2^{k^{\prime}} \int_{0}^{1}\left\|\left(\nabla_{L} F\right)_{k^{\prime}}\right\|_{L_{x}^{2}}\left\|G_{k^{\prime \prime}}\right\|_{L_{x}^{2}} d t \\
& {\left[\nabla \mathbf{F B}^{-1}\right] \quad \lesssim \sum_{k^{\prime}<k \leq k^{\prime \prime}} 2^{k^{\prime}-k^{\prime \prime}}\left\|\left(\nabla_{L} F\right)_{k^{\prime}}\right\|_{L_{t}^{2} L_{x}^{2}}\left\|\nabla G_{k^{\prime \prime}}\right\|_{L_{t}^{2} L_{x}^{2}}}
\end{aligned}
$$

Therefore,

$$
\begin{aligned}
\sum_{k}\left\|A_{k}\right\|_{L_{t}^{\infty} L_{x}^{2}} & \lesssim \sum_{k^{\prime}<k \leq k^{\prime \prime}} 2^{k^{\prime}-k^{\prime \prime}}\left\|\left(\nabla_{L} F\right)_{k^{\prime}}\right\|_{L_{t}^{2} L_{x}^{2}}\left\|\nabla g_{k^{\prime \prime}}\right\|_{L_{t}^{2} L_{x}^{2}} \\
& \lesssim \sum_{k^{\prime}<k^{\prime \prime}} 2^{\frac{k^{\prime}-k^{\prime \prime}}{2}}\left\|\left(\nabla_{L} F\right)_{k^{\prime}}\right\|_{L_{t}^{2} L_{x}^{2}}\left\|\nabla G_{k^{\prime \prime}}\right\|_{L_{t}^{2} L_{x}^{2}} \lesssim \mathcal{N}_{1}[F] \cdot \mathcal{N}_{1}[G]
\end{aligned}
$$

Remark 8.2. It is easy to see that we don't need (94); indeed we can replace it with the weak Bernstein inequalities for $L^{p^{\prime}}, L^{p^{\prime \prime}}$, with $p^{-1}+p^{\prime \prime-1}=2^{-1}$ and $p^{\prime \prime}<<p^{\prime}$, as follows

$$
\begin{aligned}
\left\|A_{k}\right\|_{L_{t}^{\infty} L_{x}^{2}} & \lesssim \sum_{k^{\prime}<k \leq k^{\prime \prime}} \int_{0}^{1}\left\|\left(\nabla_{L} F\right)_{k^{\prime}} \cdot G_{k^{\prime \prime}}\right\|_{L_{x}^{2}} d t \\
{[\mathbf{H o ̈}] } & \lesssim \sum_{k^{\prime}<k \leq k^{\prime \prime}} \int_{0}^{1}\left\|\left(\nabla_{L} F\right)_{k^{\prime}}\right\|_{L_{x}^{p^{\prime}}}\left\|G_{k^{\prime \prime}}\right\|_{L_{x}^{p^{\prime \prime}}} d t \\
{[\mathbf{w B}] } & \lesssim \sum_{k^{\prime}<k \leq k^{\prime \prime}} 2^{k^{\prime}\left(1-\frac{2}{p^{\prime}}\right)} 2^{k^{\prime \prime}\left(1-\frac{2}{p^{\prime \prime}}\right)} \int_{0}^{1}\left\|\left(\nabla_{L} F\right)_{k^{\prime}}\right\|_{L_{x}^{2}}\left\|G_{k^{\prime \prime}}\right\|_{L_{x}^{2}} d t \\
{\left[\nabla \mathbf{F B}^{-1}\right] } & \lesssim \sum_{k^{\prime}<k \leq k^{\prime \prime}} 2^{\left(k^{\prime}-k^{\prime \prime}\right) \cdot \frac{2}{p^{\prime \prime}}}\left\|\left(\nabla_{L} F\right)_{k^{\prime}}\right\|_{L_{t}^{2} L_{x}^{2}}\left\|\nabla G_{k^{\prime \prime}}\right\|_{L_{t}^{2} L_{x}^{2}}
\end{aligned}
$$

The proof then follows as before.

\subsection{Estimates for $D_{k}=P_{k} \int_{0}^{t}\left(\nabla_{L} F\right)_{\geq k} \cdot G_{\geq k}$.}

We write, $D_{k}=D_{k}^{1}+D_{k}^{2}$ where,

$$
D_{k}^{1}=\sum_{k \leq k^{\prime} \leq k^{\prime \prime}} P_{k} \int_{0}^{t}\left(\nabla_{L} F\right)_{k^{\prime}} \cdot G_{k^{\prime \prime}}, \quad D_{k}^{2}=\sum_{k \leq k^{\prime}<k^{\prime \prime}} P_{k} \int_{0}^{t}\left(\nabla_{L} F\right)_{k^{\prime \prime}} \cdot G_{k^{\prime}}
$$

We only provide the proof for the term $D_{k}^{2}$ which is more difficult to treat since the $\nabla_{L}$ derivative there falls on the factor with a higher frequency. The term $D_{k}^{1}$ can be treated in the same way without the integration by parts.

We need to apply the integration by parts estimate of proposition 7.1 followed by the scalar dual strong Bernstein inequality, Hölder, finite band and the property of 
envelopes,

$$
\mathcal{N}_{1}\left[F_{m}\right] \lesssim 2^{\epsilon|m-l|} \mathcal{N}_{1}\left[F_{l}\right], \quad \forall m, l \geq 0
$$

Thus, writing

$$
\left(\nabla_{L} F\right)_{k^{\prime \prime}} \cdot G_{k^{\prime}}=" \nabla_{L} "\left(F_{k^{\prime \prime}} \cdot G_{k^{\prime}}\right)-F_{k^{\prime \prime}} \cdot\left(\nabla_{L} G\right)_{k^{\prime}} .
$$

we derive, for $\sigma>\epsilon$,

$$
\begin{aligned}
\left\|D_{k}^{2}\right\|_{L_{t}^{\infty} L_{x}^{2}} & \lesssim \sum_{k \leq k^{\prime}<k^{\prime \prime}}\left\|P_{k} \int_{0}^{t} F_{k^{\prime \prime}} \cdot\left(\nabla_{L} G\right)_{k^{\prime}}\right\|_{L_{t}^{\infty} L_{x}^{2}} \quad \text { [prop. 7.1] } \\
& +\sum_{k \leq k^{\prime}<k^{\prime \prime}} 2^{-\sigma\left(\left|k^{\prime}-k^{\prime \prime}\right|+\left|k-k^{\prime}\right|\right)} \mathcal{N}_{1}\left[F_{k^{\prime \prime}}\right] \cdot \mathcal{N}_{1}\left[G_{k^{\prime}}\right] \\
{[\mathbf{s s B}] \&[\mathbf{E n v}] } & \lesssim 2^{k} \sum_{k \lesssim k^{\prime}<k^{\prime \prime}}\left\|\int_{0}^{t} F_{k^{\prime \prime}} \cdot\left(\nabla_{L} G\right)_{k^{\prime}}\right\|_{L_{t}^{\infty} L_{x}^{1}}+\mathcal{N}_{1}\left[F_{k}\right] \cdot \mathcal{N}_{1}\left[G_{k}\right] \\
{[\text { Hö] }] } & \lesssim 2^{k} \sum_{k \lesssim k^{\prime}<k^{\prime \prime}}\left\|F_{k^{\prime \prime}}\right\|_{L_{t}^{2} L_{x}^{2}}\left\|\left(\nabla_{L} G\right)_{k^{\prime}}\right\|_{L_{t}^{2} L_{x}^{2}}+\mathcal{N}_{1}\left[F_{k}\right] \cdot \mathcal{N}_{1}\left[G_{k}\right] \\
{[\text { Env }] } & \lesssim \sum_{k \lesssim k^{\prime}<k^{\prime \prime}} 2^{k-k^{\prime \prime}} \mathcal{N}_{1}\left[F_{k^{\prime \prime}}\right] \cdot \mathcal{N}_{1}\left[G_{k^{\prime}}\right]+\mathcal{N}_{1}\left[F_{k}\right] \cdot \mathcal{N}_{1}\left[G_{k}\right] \lesssim \mathcal{N}_{1}\left[F_{k}\right] \cdot \mathcal{N}_{1}\left[G_{k}\right]
\end{aligned}
$$

Thus,

$$
\sum_{k}\left\|D_{k}^{2}\right\|_{L_{t}^{\infty} L_{x}^{2}} \lesssim \sum_{k} \mathcal{N}_{1}\left[F_{k}\right] \cdot \mathcal{N}_{1}\left[G_{k}\right] \lesssim \mathcal{N}_{1}[F] \cdot \mathcal{N}_{1}[G]
$$

8.4. Estimates for $B_{k}=P_{k} \int_{0}^{t}\left(\nabla_{L} F\right)_{\geq k} \cdot G_{<k}$.

We start by decomposing,

$$
B_{k}=\sum_{k^{\prime}<k \leq k^{\prime \prime}} P_{k} \int_{0}^{t}\left(\nabla_{L} F\right)_{k^{\prime \prime}} \cdot G_{k^{\prime}}
$$

Integrating by parts with the help of proposition 7.1 we obtain,

$$
\begin{aligned}
\left\|B_{k}\right\|_{L_{t}^{\infty} L_{x}^{2}} & \lesssim \sum_{k^{\prime}<k \leq k^{\prime \prime}}\left(\left\|\int_{\gamma_{t}} F_{k^{\prime \prime}} \cdot\left(\nabla_{L} G\right)_{k^{\prime}}\right\|_{L_{t}^{\infty} L_{x}^{2}}+2^{-\sigma\left(\left|k^{\prime}-k^{\prime \prime}\right|+\left|k-k^{\prime}\right|\right)} \mathcal{N}_{1}\left[F_{k^{\prime \prime}}\right] \cdot \mathcal{N}_{1}\left[G_{k^{\prime}}\right]\right) \\
& \lesssim \sum_{k^{\prime}<k \leq k^{\prime \prime}}\left\|\int_{0}^{t} F_{k^{\prime \prime}} \cdot\left(\nabla_{L} G\right)_{k^{\prime}}\right\|_{L_{t}^{\infty} L_{x}^{2}}+\mathcal{N}_{1}\left[F_{k}\right] \cdot \mathcal{N}_{1}\left[G_{k}\right]
\end{aligned}
$$

To estimate the sum on the right hand side we proceed exactly as in the proof for $A_{k}$.

Estimates for $C_{k}=P_{k} \int_{0}^{t}\left(\nabla_{L} F\right)_{<k} \cdot G_{<k} \quad$ This term, which is absent in the classical paradifferential calculus, is by far the most difficult and requires a lot more 
work than the previous ones. We further decompose

$$
\begin{aligned}
& C_{k}=C_{k}^{(1)}+C_{k}^{(2)}, \\
& C_{k}^{(1)}=\sum_{k^{\prime} \leq k^{\prime \prime}<k} P_{k} \int_{0}^{t}\left(\nabla_{L} F\right)_{k^{\prime}} \cdot G_{k^{\prime \prime}}, \\
& C_{k}^{(2)}=\sum_{k^{\prime}<k^{\prime \prime}<k} P_{k} \int_{0}^{t}\left(\nabla_{L} F\right)_{k^{\prime \prime}} \cdot G_{k^{\prime}}
\end{aligned}
$$

As before we ignore the term $C_{k}^{(1)}$ since the term $C_{k}^{(2)}$ is clearly the more difficult one as the $\nabla_{L}$ derivative falls on a term with higher frequency and thus requires an integration by parts.

We first integrate by parts with the help of proposition 7.1.

$$
\left\|C_{k}^{(2)}\right\|_{L_{t}^{\infty} L_{x}^{2}} \lesssim \sum_{k^{\prime}<k^{\prime \prime}<k}\left\|P_{k} \int_{0}^{t} F_{k^{\prime \prime}} \cdot\left(\nabla_{L} G\right)_{k^{\prime}}\right\|_{L_{t}^{\infty} L_{x}^{2}}+\mathcal{N}_{1}\left[F_{k}\right] \cdot \mathcal{N}_{1}\left[G_{k}\right]
$$

To control the first term above we need to use first the finite band condition $\left[\nabla \mathbf{F B}^{-\mathbf{1}}\right]$ followed by Lemma 3.11 and the Leibnitz rule

$$
\begin{aligned}
\left\|P_{k} \int_{0}^{t} F_{k^{\prime \prime}} \cdot\left(\nabla_{L} G\right)_{k^{\prime}}\right\|_{L_{t}^{\infty} L_{x}^{2}} & \lesssim 2^{-k}\left\|\nabla \int_{0}^{t} F_{k^{\prime \prime}} \cdot\left(\nabla_{L} G\right)_{k^{\prime}}\right\|_{L_{t}^{\infty} L_{x}^{2}} \\
{[\text { Le 3.11 }] } & \lesssim 2^{-k}\left(\left\|\nabla\left(F_{k^{\prime \prime}} \cdot\left(\nabla_{L} G\right)_{k^{\prime}}\right)\right\|_{L_{t}^{1} L_{x}^{2}}+\left\|F_{k^{\prime \prime}} \cdot\left(\nabla_{L} G\right)_{k^{\prime}}\right\|_{L_{t}^{1} L_{x}^{2}}\right) \\
{[\text { Leib }] } & \lesssim 2^{-k}\left\|\nabla F_{k^{\prime \prime}} \cdot\left(\nabla_{L} G\right)_{k^{\prime}}\right\|_{L_{t}^{1} L_{x}^{2}}+2^{-k}\left\|F_{k^{\prime \prime}} \cdot \nabla\left(\nabla_{L} G\right)_{k^{\prime}}\right\|_{L_{t}^{1} L_{x}^{2}} \\
& +2^{-k}\left\|F_{k^{\prime \prime}} \cdot\left(\nabla_{L} G\right)_{k^{\prime}}\right\|_{L_{t}^{1} L_{x}^{2}}
\end{aligned}
$$

To continue it suffices to consider only the first term since in that case the derivative falls on a term with higher frequency. Using Hölder followed by the inequality (86) of Lemma 5.6 and the weak Bernstein inequality, we infer that,

$$
\begin{aligned}
\left\|\nabla F_{k^{\prime \prime}} \cdot\left(\nabla_{L} G\right)_{k^{\prime}}\right\|_{L_{t}^{1} L_{x}^{2}} & \lesssim\left\|\nabla F_{k^{\prime \prime}}\right\|_{L_{t}^{2} L_{x}^{4}}\left\|\left(\nabla_{L} G\right)_{k^{\prime}}\right\|_{L_{t}^{2} L_{x}^{4}} \\
{[(86)] } & \lesssim 2^{\frac{k^{\prime \prime}+k^{\prime}}{2}} \mathcal{N}_{1}\left[F_{k^{\prime \prime}}\right] \cdot \mathcal{N}_{1}\left[G_{k^{\prime}}\right]
\end{aligned}
$$

This leads to the estimate

$$
\begin{aligned}
\sum_{k} \sum_{k^{\prime}<k^{\prime \prime}<k}\left\|P_{k} \int_{0}^{t} F_{k^{\prime \prime}} \cdot\left(\nabla_{L} G\right)_{k^{\prime}}\right\|_{L_{t}^{\infty} L_{x}^{2}} & \lesssim \sum_{k} \sum_{k^{\prime}<k^{\prime \prime}<k} 2^{\frac{k^{\prime \prime}+k^{\prime}}{2}-k} \mathcal{N}_{1}\left[F_{k^{\prime \prime}}\right] \cdot \mathcal{N}_{1}\left[G_{k^{\prime}}\right] \\
& \lesssim \mathcal{N}_{1}[F] \cdot \mathcal{N}_{1}[G]
\end{aligned}
$$

\section{INTEGRATED SHARP PRODUCT ESTIMATE I}

In this section we prove the integrated sharp product estimate (79) of theorem 4.9. We have already shown that it suffices to prove the estimate only in a fully scalar case. Namely, that for two scalar functions $f$ and $g$,

$$
\left\|\int_{0}^{t} f \cdot g\right\|_{\mathcal{B}^{0}} \lesssim\|f\|_{\mathcal{P}^{0}} \cdot\left(\mathcal{N}_{1}[g]+\|g\|_{L_{x}^{\infty} L_{t}^{2}}\right)
$$


Observe that it suffices to prove,

$$
\sum_{k \geq 0}\left\|P_{k} \int_{0}^{t} f \cdot g\right\|_{L_{t}^{\infty} L_{x}^{2}} \lesssim\|f\|_{\mathcal{P}^{0}} \cdot\left(\mathcal{N}_{1}[g]+\|g\|_{L_{x}^{\infty} L_{t}^{2}}\right)
$$

Remark 9.1. The proof below will show that we have, in fact, a stronger dyadic estimate: for all $k \geq 0$,

$\left\|P_{k} \int_{0}^{t} f \cdot g\right\|_{L_{t}^{\infty} L_{x}^{2}} \lesssim\left(\mathcal{N}_{1}[g]+\|g\|_{L_{x}^{\infty} L_{t}^{2}}\right) \cdot\left(\sum_{k^{\prime}} 2^{-\left|k-k^{\prime}\right|}\left\|P_{k^{\prime}} f\right\|_{L_{t}^{2} L_{x}^{2}}+2^{-k}\|f\|_{L_{t}^{2} L_{x}^{2}}\right)$,

needed to establish the second part of theorem 4.3.

We start by applying the LP-decomposition to $f \cdot g$.

$$
f \cdot g=f_{<k} \cdot g+f_{\geq k} \cdot g
$$

Thus,

$$
P_{k}\left(\int_{0}^{t} f \cdot g\right)=A_{k}+B_{k}
$$

where

$$
A_{k}=P_{k} \int_{0}^{t} f_{<k} \cdot g, \quad B_{k}=P_{k} \int_{0}^{t} f_{\geq k} \cdot g
$$

9.2. Estimates for $A_{k}=P_{k} \int_{0}^{t} f_{<k} \cdot g$.

Applying the dual finite band condition followed by (31) of Lemma 3.11 and the Leibnitz rule we obtain

$$
\begin{aligned}
\left\|A_{k}\right\|_{L_{t}^{\infty} L_{x}^{2}} & \lesssim 2^{-k}\left\|\nabla \int_{0}^{t} f_{<k} \cdot g\right\|_{L_{t}^{\infty} L_{x}^{2}} \\
& \lesssim 2^{-k}\left\|\nabla\left(f_{<k} \cdot g\right)\right\|_{L_{x}^{2} L_{t}^{1}} \\
{[\mathbf{L e i b}] } & \lesssim 2^{-k}\left\|\nabla f_{<k} \cdot g\right\|_{L_{x}^{2} L_{t}^{1}}+2^{-k}\left\|f_{<k} \cdot \nabla g\right\|_{L_{x}^{2} L_{t}^{1}} \\
{[\mathbf{H} \ddot{\mathbf{o}}] } & \lesssim 2^{-k}\left\|\nabla f_{<k}\right\|_{L_{x}^{2} L_{t}^{2}}\|g\|_{L_{x}^{\infty} L_{t}^{2}}+2^{-k}\left\|f_{<k}\right\|_{L_{t}^{2} L_{x}^{\infty}}\|\nabla g\|_{L_{t}^{2} L_{x}^{2}} \\
{[\mathbf{F B}] \&[\mathbf{s s B}] } & \lesssim \sum_{k^{\prime}<k} 2^{k^{\prime}-k}\left\|f_{k^{\prime}}\right\|_{L_{t}^{2} L_{x}^{2}}\left(\|g\|_{L_{x}^{\infty} L_{t}^{2}}+\|\nabla g\|_{L_{t}^{2} L_{x}^{2}}\right)
\end{aligned}
$$

Therefore,

$$
\begin{aligned}
\sum_{k}\left\|A_{k}\right\|_{L_{t}^{\infty} L_{x}^{2}} & \lesssim\left(\|g\|_{L_{x}^{\infty} L_{t}^{2}}+\|\nabla g\|_{L_{t}^{2} L_{x}^{2}}\right) \sum_{k} \sum_{k^{\prime}<k} 2^{k^{\prime}-k}\left\|f_{k^{\prime}}\right\|_{L_{t}^{2} L_{x}^{2}} \\
& \lesssim\left(\|g\|_{L_{x}^{\infty} L_{t}^{2}}+\|\nabla g\|_{L_{t}^{2} L_{x}^{2}}\right) \sum_{k^{\prime}}\left\|f_{k^{\prime}}\right\|_{L_{t}^{2} L_{x}^{2}} \\
& \lesssim\|f\|_{\mathcal{P}^{0}} \cdot\left(\|g\|_{L_{x}^{\infty} L_{t}^{2}}+\|\nabla g\|_{L_{t}^{2} L_{x}^{2}}\right)
\end{aligned}
$$


9.3. Estimates for $B_{k}=P_{k} \int_{0}^{t} f_{\geq k} \cdot g$. We decompose $f_{\geq k}=\sum_{k^{\prime} \geq k} f_{k^{\prime}}$ and on each $k^{\prime \prime}$ dyadic LP-piece apply the finite band condition $f_{k^{\prime}} \approx 2^{-2 k^{\prime}} \Delta f_{k^{\prime}}$.

$$
\begin{aligned}
\left\|B_{k}\right\|_{L_{t}^{\infty} L_{x}^{2}} & \lesssim\left\|P_{k} \int_{0}^{t} f_{\geq k} \cdot g\right\|_{L_{t}^{\infty} L_{x}^{2}} \lesssim \sum_{k^{\prime} \geq k}\left\|P_{k} \int_{0}^{t} f_{k^{\prime}} \cdot g\right\|_{L_{t}^{\infty} L_{x}^{2}} \\
{\left[\Delta \mathbf{F B}^{-1}\right] } & \lesssim \sum_{k^{\prime} \geq k} 2^{-2 k^{\prime}}\left\|P_{k} \int_{0}^{t} \Delta f_{k^{\prime}} \cdot g\right\|_{L_{t}^{\infty} L_{x}^{2}} \\
& \lesssim \sum_{k^{\prime} \geq k} 2^{-2 k^{\prime}}\left\|P_{k} \int_{0}^{t} \operatorname{div}\left(\nabla f_{k^{\prime}} \cdot g\right)\right\|_{L_{t}^{\infty} L_{x}^{2}} \\
& +\sum_{k^{\prime} \geq k} 2^{-2 k^{\prime}}\left\|P_{k} \int_{0}^{t} \nabla f_{k^{\prime}} \cdot \nabla g\right\|_{L_{t}^{\infty} L_{x}^{2}}=B_{k}^{(1)}+B_{k}^{(2)}
\end{aligned}
$$

To estimate $B_{k}^{(1)}$ we use a result of Lemma 3.13 according to which for any vectorfield $F$

$$
\int_{0}^{t} \operatorname{div} F=\operatorname{div} W+E
$$

where $W$ is a solution of the transport equation

$$
\nabla_{L} W-\chi \cdot W=F,\left.\quad W\right|_{S_{0}}=0
$$

and the error term $E$ satisfies the estimate

$$
\|E\|_{L_{x}^{\frac{2 p}{2-p}} L_{t}^{\infty}} \lesssim\|F\|_{L_{x}^{p} L_{t}^{1}}
$$

for any $1 \leq p \leq 2$. Note that for any $r \geq 1$ we have the following estimate for $W$ :

$$
\|W\|_{L_{x}^{r} L_{t}^{\infty}} \lesssim\|F\|_{L_{x}^{r} L_{t}^{1}}
$$

Therefore we use the representation (97) with $F=\nabla f_{k^{\prime}} \cdot g$, apply the dual finite band condition together with strong scalar Bernstein inequality followed by the estimates (98) and (99) with $p=1$ and $r=2$, and finite band condition.

$$
\begin{aligned}
B_{k}^{(1)} & \lesssim \sum_{k^{\prime} \geq k} 2^{-2 k^{\prime}}\left\|P_{k} \operatorname{div} W\right\|_{L_{t}^{\infty} L_{x}^{2}}+\sum_{k^{\prime} \geq k} 2^{-2 k^{\prime}}\left\|P_{k} E\right\|_{L_{t}^{\infty} L_{x}^{2}} \\
{[\mathbf{F B} \nabla] \&\left[\mathbf{s s B}^{*}\right] } & \lesssim \sum_{k^{\prime} \geq k} 2^{k-2 k^{\prime}}\|W\|_{L_{t}^{\infty} L_{x}^{2}}+\sum_{k^{\prime} \geq k} 2^{k-2 k^{\prime}}\|E\|_{L_{t}^{\infty} L_{x}^{1}} \\
& \lesssim \sum_{k^{\prime} \geq k} 2^{k-2 k^{\prime}}\left\|\nabla f_{k^{\prime}} \cdot g\right\|_{L_{x}^{2} L_{t}^{1}}+\sum_{k^{\prime} \geq k} 2^{k-2 k^{\prime}}\left\|\nabla f_{k^{\prime}} \cdot g\right\|_{L_{x}^{2} L_{t}^{1}} \\
& \lesssim \sum_{k^{\prime} \geq k} 2^{k-2 k^{\prime}}\left\|\nabla f_{k^{\prime}}\right\|_{L_{x}^{2} L_{t}^{2}}\|g\|_{L_{x}^{\infty} L_{t}^{2}} \\
{[\nabla \mathbf{F B}] } & \lesssim \sum_{k^{\prime \prime} \geq k} 2^{k-k^{\prime}}\left\|f_{k^{\prime}}\right\|_{L_{t}^{2} L_{x}^{2}}\|g\|_{L_{x}^{\infty} L_{t}^{2}}
\end{aligned}
$$

Thus,

$$
\sum_{k} B_{k}^{(1)} \lesssim \sum_{k} \sum_{k^{\prime} \geq k} 2^{k-k^{\prime}}\left\|f_{k^{\prime}}\right\|_{L_{t}^{2} L_{x}^{2}}\|g\|_{L_{x}^{\infty} L_{t}^{2}} \lesssim\|f\|_{\mathcal{P}^{0}}\|g\|_{L_{x}^{\infty} L_{t}^{2}}
$$


We now estimate the term $B_{k}^{(2)}$. Using the dual strong scalar Bernstein inequality followed by Hölder inequality and the finite band property, we obtain

$$
\begin{aligned}
B_{k}^{(2)} & \lesssim \sum_{k^{\prime} \geq k} 2^{k-2 k^{\prime}}\left\|\int_{0}^{t} \nabla f_{k^{\prime}} \cdot \nabla g\right\|_{L_{t}^{\infty} L_{x}^{1}} \\
& \lesssim \sum_{k^{\prime} \geq k} 2^{k-2 k^{\prime}}\left\|\nabla f_{k^{\prime}}\right\|_{L_{t}^{2} L_{x}^{2}}\|\nabla g\|_{L_{t}^{2} L_{x}^{2}} \\
{[\nabla \mathbf{F B}] } & \lesssim \sum_{k^{\prime} \geq k} 2^{k-k^{\prime}}\left\|f_{k^{\prime}}\right\|_{L_{t}^{2} L_{x}^{2}}\|g\|_{L_{t}^{2} L_{x}^{2}}
\end{aligned}
$$

Therefore, as in the case of $B_{k}^{(1)}$,

$$
\sum_{k} B_{k}^{(2)} \lesssim\|f\|_{\mathcal{P}^{0}}\|g\|_{L_{x}^{\infty} L_{t}^{2}}
$$

It then follows that

$$
\sum_{k}\left\|B_{k}\right\|_{L_{t}^{\infty} L_{x}^{2}} \lesssim\|f\|_{\mathcal{P}^{0}}\|g\|_{L_{x}^{\infty} L_{t}^{2}}
$$

\section{INTEGRATED SHARP PRODUCT ESTIMATE II}

In this section we indicate how to prove the sharp product estimate of theorem 4.4, which has already been reduced to the fully scalar case. For scalar functions $w, g$. where $w$ is a solution of the transport equation

$$
\nabla_{L} w=f,\left.\quad f\right|_{S_{0}}=f_{0},
$$

we need to prove the estimate

$$
\|w \cdot g\|_{P^{0}} \lesssim\left(\left\|f_{0}\right\|_{B_{2,1}^{0}}+\|f\|_{\mathcal{P}^{0}}\right)\left(\mathcal{N}_{1}[g]+\|g\|_{L_{x}^{\infty} L_{t}^{2}}\right)
$$

Proof : We define the functions $w_{(<k)}, w_{(k)}, w_{(\geq k)}$ as solutions of the transport equations

$$
\begin{aligned}
& \nabla_{L} w_{(<k)}=f_{<k},\left.\quad w_{(<k)}\right|_{S_{0}}=f_{0<k}, \\
& \nabla_{L} w_{(k)}=f_{<k},\left.\quad w_{(k)}\right|_{S_{0}}=f_{0 k}, \\
& \nabla_{L} w_{(\geq k)}=f_{<k},\left.\quad w_{(\geq k)}\right|_{S_{0}}=f_{0 \geq k},
\end{aligned}
$$

Observe that according to the results of lemmas 3.6 and 3.11 we have

$$
\begin{aligned}
\left\|w_{([s] k)}\right\|_{L_{x}^{p} L_{t}^{\infty}} & \lesssim\left\|f_{0[s] k}\right\|_{L_{x}^{p}}+\left\|f_{[s] k}\right\|_{L_{x}^{p} L_{t}^{1}}, \\
\left\|\nabla w_{([s] k)}\right\|_{L_{x}^{p} L_{t}^{\infty}} & \lesssim\left\|\nabla f_{0[s] k}\right\|_{L_{x}^{p}}+\left\|\nabla f_{[s] k}\right\|_{L_{x}^{p} L_{t}^{1}}
\end{aligned}
$$

where $[s] k=<k, k, \geq k$. We now consider the following LP-decomposition of $F \cdot G$ :

$$
w \cdot g=w_{(<k)} \cdot g+w_{(\geq k)} \cdot g
$$

Thus, $P_{k}(w \cdot g)=A_{k}+B_{k}$, where

$$
A_{k}=P_{k}\left(w_{(<k)} \cdot g\right), \quad B_{k}=P_{k}\left(w_{(\geq k)} \cdot f\right) .
$$


10.1. Estimates for $A_{k}=P_{k}\left(w_{(<k)} \cdot g\right)$.

We have

$$
\begin{aligned}
\left\|A_{k}\right\|_{L_{t}^{2} L_{x}^{2}} & \lesssim 2^{-k}\left\|\nabla\left(w_{(<k)} \cdot g\right)\right\|_{L_{t}^{2} L_{x}^{2}} \\
{[\mathbf{L e i b}] } & \lesssim 2^{-k}\left\|\nabla w_{(<k)} \cdot g\right\|_{L_{x}^{2} L_{t}^{2}}+2^{-k}\left\|w_{(<k)} \cdot \nabla g\right\|_{L_{x}^{2} L_{t}^{2}} \\
{[\mathbf{H o ̈}] } & \lesssim 2^{-k}\left\|\nabla w_{(<k)}\right\|_{L_{x}^{2} L_{t}^{\infty}}\|g\|_{L_{x}^{\infty} L_{t}^{2}}+2^{-k}\left\|w_{(<k)}\right\|_{L_{t}^{\infty} L_{x}^{\infty}}\|\nabla g\|_{L_{t}^{2} L_{x}^{2}} \\
& \lesssim 2^{-k} \sum_{k^{\prime}<k}\left\|\nabla w_{\left(k^{\prime}\right)}\right\|_{L_{x}^{2} L_{t}^{\infty}}\|g\|_{L_{x}^{\infty} L_{t}^{2}}+2^{-k}\left\|w_{\left(k^{\prime}\right)}\right\|_{L_{t}^{\infty} L_{x}^{\infty}}\|\nabla g\|_{L_{t}^{2} L_{x}^{2}} \\
{[(101)] } & \lesssim 2^{-k} \sum_{k^{\prime}<k}\left(\left\|\nabla f_{0_{k^{\prime}}}\right\|_{L_{x}^{2}}+\left\|\nabla f_{k^{\prime}}\right\|_{L_{x}^{2} L_{t}^{1}}\right)\|g\|_{L_{x}^{\infty} L_{t}^{2}} \\
& +2^{-k}\left(\left\|f_{0_{k^{\prime}}}\right\|_{L_{x}^{\infty}}+\left\|f_{k^{\prime}}\right\|_{L_{x}^{\infty} L_{t}^{1}}\right)\|\nabla g\|_{L_{t}^{2} L_{x}^{2}} \\
{[\nabla \mathbf{F B}] \&[\mathbf{s s B}] } & \lesssim \sum_{k^{\prime}<k} 2^{k^{\prime}-k}\left(\left\|f_{0_{k^{\prime}}}\right\|_{L_{x}^{2}}+\left\|f_{k^{\prime}}\right\|_{L_{t}^{2} L_{x}^{2}}\right)\left(\|g\|_{L_{x}^{\infty} L_{t}^{2}}+\|\nabla g\|_{L_{t}^{2} L_{x}^{2}}\right)
\end{aligned}
$$

Therefore,

$$
\sum_{k} \lesssim\left\|A_{k}\right\|_{L_{t}^{\infty} L_{x}^{2}} \lesssim\left(\left\|f_{0}\right\|_{B_{2,1}^{0}}+\|f\|_{\mathcal{P}^{0}}\right) \cdot\left(\|g\|_{L_{x}^{\infty} L_{t}^{2}}+\|\nabla g\|_{L_{t}^{2} L_{x}^{2}}\right)
$$

10.2. Estimates for $B_{k}=P_{k}\left(w_{(\geq k)} \cdot g\right)$.

We define the functions $[\Delta w]_{\left(k^{\prime}\right)}$ as solutions of the transport equations

$$
\nabla_{L}[\Delta w]_{\left(k^{\prime}\right)}=\Delta f_{k^{\prime}},\left.\quad[\Delta w]_{\left(k^{\prime}\right)}\right|_{S_{0}}=\Delta f_{0 k^{\prime}}
$$

Observe that $\Delta f_{k^{\prime}} \approx 2^{2 k^{\prime}} f_{k^{\prime}}$ and therefore $[\Delta w]_{\left(k^{\prime}\right)} \approx 2^{2 k^{\prime}} w_{\left(k^{\prime}\right)}$. In view of this,

$$
\left\|B_{k}\right\|_{L_{t}^{2} L_{x}^{2}} \lesssim \sum_{k^{\prime} \geq k} 2^{-2 k^{\prime}}\left\|P_{k}\left([\Delta w]_{\left(k^{\prime}\right)} \cdot g\right)\right\|_{L_{t}^{2} L_{x}^{2}}
$$

Now, according to lemma 3.13 for $p=1$, we can write

$$
[\Delta w]_{\left(k^{\prime}\right)}=\operatorname{div} W_{\left(k^{\prime}\right)}+E_{\left(k^{\prime}\right)}
$$

where,

$$
\begin{aligned}
\nabla_{L} W_{\left(k^{\prime}\right)}-\chi \cdot W_{\left(k^{\prime}\right)} & =\nabla f_{k^{\prime}},\left.\quad W_{\left(k^{\prime}\right)}\right|_{S_{0}}=\nabla\left(f_{0}\right)_{k^{\prime}} \\
\left\|E_{\left(k^{\prime}\right)}\right\|_{L_{x}^{2} L_{t}^{\infty}} & \lesssim\left\|\nabla\left(f_{0}\right)_{k^{\prime}}\right\|_{L_{2}\left(S_{0}\right)}+\left\|\nabla f_{k^{\prime}}\right\|_{L_{x}^{2} L_{t}^{1}}
\end{aligned}
$$

The proof continues essentially as in the proof of the estimates for $B_{k}$ in the previous section.

\section{Sharp scalar Commutator estimates}

The goal of this section is to prove the last part of theorem 4.9. In other words we have to show that any solution $f$ of a homogeneous scalar transport equation

$$
\nabla_{L} f=0,\left.\quad f\right|_{S_{0}}=f_{0}
$$


verifies the estimate

$$
\|f\|_{\mathcal{B}^{0}} \lesssim\left\|f_{0}\right\|_{B_{2,1}^{0}\left(S_{0}\right)}
$$

We first observe that it is trivial to take care of the low frequency component $\left\|P_{<0} f\right\|_{L_{t}^{\infty} L_{x}^{2}}$ of $\|f\|_{\mathcal{B}^{0}}$. Indeed, $\left\|P_{<0} f\right\|_{L_{t}^{\infty} L_{x}^{2}} \lesssim\|f\|_{L_{t}^{\infty} L_{x}^{2}}$ and clearly $\|f\|_{L_{t}^{\infty} L_{x}^{2}} \leq$ $\left\|f_{0}\right\|_{L^{2}} \lesssim\left\|f_{0}\right\|_{B_{2,1}^{0}\left(S_{0}\right)}$. Therefore we only have to show that,

$$
\sum_{k \geq 0}\left\|P_{k} f\right\|_{L_{t}^{\infty} L_{x}^{2}} \lesssim\left\|f_{0}\right\|_{B_{2,1}^{0}\left(S_{0}\right)}
$$

Commuting the equation with the LP-projection we obtain

$$
\nabla_{L} P_{k} f=\left[\nabla_{L}, P_{k}\right] f,\left.\quad\left(P_{k} f\right)\right|_{S_{0}}=P_{k} f_{0}
$$

Thus, $P_{k} f=P_{k} f_{0}+\int_{0}^{t}\left[\nabla_{L}, P_{k}\right] f$ and,

$$
\left\|P_{k} f(t)\right\|_{L_{t}^{\infty} L_{x}^{2}} \lesssim\left\|P_{k} f_{0}\right\|_{L^{2}\left(S_{0}\right)}+\left\|\int_{0}^{t}\left[\nabla_{L}, P_{k}\right] f\right\|_{L_{t}^{\infty} L_{x}^{2}}
$$

Remark 11.1. During the argument below we need to keep track of the intervals of integration in $t$. For this reason we denote by $\|f(t)\|_{L_{t}^{q} L_{x}^{p}}$ spacetime norms in the interval $[0, t]$. Often though, when no confusion is possible, we will drop the explicit dependence on $t$.

Consequently,

$$
\sum_{k \geq 0}\left\|P_{k} f(t)\right\|_{L_{t}^{\infty} L_{x}^{2}} \lesssim\left\|f_{0}\right\|_{B_{2,1}^{0}\left(S_{0}\right)}+\sum_{k \geq 0}\left\|\int_{0}^{t}\left[\nabla_{L}, P_{k}\right] f(t)\right\|_{L_{t}^{\infty} L_{x}^{2}}
$$

In the proposition below we shall prove the following inequality:

$\sum_{k \geq 0}\left\|\int_{0}^{t}\left[\nabla_{L}, P_{k}\right] f(t)\right\|_{L_{t}^{\infty} L_{x}^{2}} \lesssim \Delta_{0} \sum_{k \geq 0}\left\|P_{k} f(t)\right\|_{L_{t}^{\infty} L_{x}^{2}}+\int_{0}^{t} \sum_{k \geq 0}\left\|P_{k} f(s)\right\|_{L_{t}^{\infty} L_{x}^{2}} d s$.

Thus, for small $\Delta_{0}$,

$$
\sum_{k \geq 0}\left\|P_{k} f(t)\right\|_{L_{t}^{\infty} L_{x}^{2}} \lesssim\left\|f_{0}\right\|_{B_{2,1}^{0}\left(S_{0}\right)}+\int_{0}^{t} \sum_{k \geq 0}\left\|P_{k} f(s)\right\|_{L_{t}^{\infty} L_{x}^{2}} d s .
$$

and the desired estimate follows by a straightforward Gronwall inequality. Therefore to prove (104) it suffices to establish the following:

Proposition 11.2. Let $f$ be a scalar function on $\mathcal{H}$. Then

$$
\sum_{k \geq 0}\left\|\int_{0}^{t}\left[\nabla_{L}, P_{k}\right] f\right\|_{L_{t}^{\infty} L_{x}^{2}} \leq \epsilon\|f\|_{\mathcal{B}^{0}}+\int_{0}^{t}\|f(s)\|_{B^{0}} d s
$$

where $\|f(s)\|_{\mathcal{B}^{0}}=\sum_{k \geq 0}\left\|P_{k} f(s)\right\|_{L_{t}^{\infty} L_{x}^{2}}+\left\|P_{<0} f(s)\right\|_{L_{t}^{\infty} L_{x}^{2}}$ in the spirit of the remark above.

Proof : $\quad$ Since $P_{k}=\int_{0}^{\infty} m_{k}(\tau) U(\tau) f$ we have,

$$
\int_{0}^{t}\left[\nabla_{L}, P_{k}\right] f=\int_{0}^{\infty} m_{k}(\tau) d \tau \int_{0}^{t}\left[\nabla_{L}, U(\tau)\right] f d s
$$


or, introducing the notation

$$
\mathcal{L}(\tau) f=\int_{0}^{t}\left[\nabla_{L}, U(\tau)\right] f
$$

we have,

$$
\begin{aligned}
\int_{0}^{t}\left[\nabla_{L}, P_{k}\right] f & =\int_{0}^{\infty} m_{k}(\tau) \mathcal{L}(\tau) f d \tau \\
& =\int_{0}^{1} m_{k}(\tau) \mathcal{L}(\tau) f d \tau+\int_{1}^{\infty} m_{k}(\tau) \mathcal{L}(\tau) f d \tau
\end{aligned}
$$

Observe that the integral $\int_{1}^{\infty} m_{k}(\tau) \mathcal{L}(\tau) f d \tau$ is a lot easier to estimate. In fact all we need to treat it is to show that $\mathcal{L}(\tau) f$ verifies the estimate,

$$
\|\mathcal{L}(\tau) f\|_{L_{t}^{\infty} L_{x}^{2}} \lesssim \tau^{K}\|f\|_{L_{t}^{2} L_{x}^{2}}, \quad \text { for } \quad \tau \geq 1
$$

for some positive value of $K$. Indeed (108) easily implies the better estimate

$$
\sum_{k}\left\|\int_{1}^{\infty} m_{k}(\tau) \mathcal{L}(\tau) f d \tau\right\|_{L_{t}^{\infty} L_{x}^{2}} \lesssim\|f\|_{L_{t}^{2} L_{x}^{2}}
$$

In what follows we shall only concentrate on the more difficult term $\int_{0}^{1} m_{k}(\tau) \mathcal{L}(\tau) f d \tau$ and ignore the contribution of $\tau \geq 1$.

We start with a simple property of a $\mathcal{B}^{0}$ function in terms of the heat semigroup $U(\tau)$.

Lemma 11.3. For any smooth scalar function $f$ on $\mathcal{H}$

$$
\int_{0}^{1}\left\|\nabla^{2} U(\tau) f\right\|_{L_{t}^{\infty} L_{x}^{2}} d \tau \lesssim\|f\|_{\mathcal{B}^{0}}
$$

Proof : First, in view of the scalar Bochner inequality (42),

$$
\left\|\nabla^{2} U(\tau) f\right\|_{L_{x}^{2}} \lesssim\|\Delta U(\tau) f\|_{L_{x}^{2}}
$$

We now decompose

$$
\|\Delta U(\tau) f\|_{L_{x}^{2}} \lesssim \sum_{k \geq 0}\left\|\Delta P_{k} U(\tau) f\right\|_{L_{x}^{2}}+\|f\|_{L_{x}^{2}}
$$

We note the following,

$$
\left\|\Delta P_{k} U(\tau) f\right\|_{L_{x}^{2}} \lesssim \min \left(2^{2 k}, 2^{-2 k} \tau^{-2}\right)\left\|P_{k} f\right\|_{L_{x}^{2}}
$$

Indeed we have both,

$$
\begin{aligned}
\left\|\Delta P_{k} U(\tau) f\right\|_{L_{x}^{2}} & \lesssim 2^{2 k}\left\|P_{k} f\right\|_{L_{x}^{2}}, \\
\left\|\Delta P_{k} U(\tau) f\right\|_{L_{x}^{2}} & \lesssim \tau^{-1}\left\|P_{k} U(\tau / 2) f\right\|_{L_{x}^{2}} \lesssim 2^{-2 k} \tau^{-2}\left\|P_{k} f\right\|_{L_{x}^{2}}
\end{aligned}
$$


Therefore,

$$
\begin{aligned}
& \int_{0}^{1}\left\|\nabla^{2} U(\tau) f\right\|_{L_{t}^{\infty} L_{x}^{2}} d \tau \lesssim \sum_{k \geq 0} \int_{0}^{1}\left\|\Delta P_{k} U(\tau) f\right\|_{L_{t}^{\infty} L_{x}^{2}} d \tau+\|f\|_{L_{x}^{2}} \\
& \lesssim \sum_{k \geq 0}\left(\int_{0}^{2^{-2 k}} 2^{2 k}\left\|P_{k} f\right\|_{L_{t}^{\infty} L_{x}^{2}} d \tau+\int_{2^{-2 k}}^{1} 2^{-2 k} \tau^{-2}\left\|P_{k} f\right\|_{L_{t}^{\infty} L_{x}^{2}} d \tau\right) \\
& \lesssim \sum_{k \geq 0}\left\|P_{k} f\right\|_{L_{t}^{\infty} L_{x}^{2}}+\|f\|_{L_{x}^{2}} \lesssim\|f\|_{\mathcal{B}^{0}}
\end{aligned}
$$

as desired.

The main step in proving the proposition is the following:

Lemma 11.4. The following estimates hold:

$$
\sup _{0 \leq \tau \leq 1}\|\mathcal{L}(\tau) f(t)\|_{L_{t}^{\infty} L_{x}^{2}} \lesssim\|f\|_{\mathcal{B}^{0}}
$$

Moreover for any $\tau \approx 2^{-2 m_{0}}$,

$$
\|\mathcal{L}(\tau) f(t)\|_{L_{t}^{\infty} L_{x}^{2}} \lesssim \sum_{k \geq 0} 2^{-\epsilon\left|m_{0}-k\right|}\left(\Delta_{0} \cdot\left\|P_{k} f(t)\right\|_{L_{t}^{\infty} L_{x}^{2}}+\int_{0}^{t}\left\|P_{k} f(s)\right\|_{L_{x}^{2}}\right)
$$

We postpone the proof of the lemma and show now how (113) implies proposition 11.2. Indeed observe that estimate (113) is equivalent to the bound,

$$
\|\mathcal{L}(\tau) f(t)\|_{L_{t}^{\infty} L_{x}^{2}} \lesssim \sum_{m} \min \left(2^{m} \tau^{\frac{1}{2}}, 2^{-m} \tau^{-\frac{1}{2}}\right)^{\epsilon}\left(\Delta_{0}\left\|P_{m} f(t)\right\|_{L_{t}^{\infty} L_{x}^{2}}+\int_{0}^{t}\left\|P_{m} f(s)\right\|_{L_{x}^{2}} d s\right)
$$

Therefore, in view of (107),

$$
\begin{aligned}
& \left\|\int_{0}^{t}\left[\nabla_{L}, P_{k}\right]\right\|_{L_{t}^{\infty} L_{x}^{2}} \lesssim \int_{0}^{1} m_{k}(\tau)\|\mathcal{L}(\tau) f(t)\|_{L_{t}^{\infty} L_{x}^{2}} \\
\lesssim & \sum_{m} \int_{0}^{1} m_{k}(\tau) \min \left(2^{m} \tau^{\frac{1}{2}}, 2^{-m} \tau^{-\frac{1}{2}}\right)^{\epsilon}\left(\Delta_{0}\left\|P_{m} f(t)\right\|_{L_{t}^{\infty} L_{x}^{2}}+\int_{0}^{t}\left\|P_{m} f\right\|_{L_{x}^{2}}\right) \\
= & \sum_{m} 2^{\epsilon m} \int_{0}^{2^{-2 m}} m_{k}(\tau) \tau^{\frac{\epsilon}{2}}\left(\Delta_{0}\left\|P_{m} f(t)\right\|_{L_{t}^{\infty} L_{x}^{2}}+\int_{0}^{t}\left\|P_{m} f\right\|_{L_{x}^{2}}\right) \\
+ & \sum_{m} 2^{-\epsilon m} \int_{2^{-2 m}}^{1} m_{k}(\tau) \tau^{-\frac{\epsilon}{2}}\left(\Delta_{0}\left\|P_{m} f(t)\right\|_{L_{t}^{\infty} L_{x}^{2}}+\int_{0}^{t}\left\|P_{m} f\right\|_{L_{x}^{2}}\right) \\
= & \sum_{m} 2^{\epsilon(m-k)} \int_{0}^{2^{2(k-m)}} \tilde{m}(\tau)\left(\Delta_{0}\left\|P_{m} f(t)\right\|_{L_{t}^{\infty} L_{x}^{2}}+\int_{0}^{t}\left\|P_{m} f\right\|_{L_{x}^{2}}\right) \\
+ & \sum_{m} 2^{\epsilon(k-m)} \int_{2^{2(k-m)}}^{2^{2 k}} \hat{m}(\tau)\left(\Delta_{0}\left\|P_{m} f(t)\right\|_{L_{t}^{\infty} L_{x}^{2}}+\int_{0}^{t}\left\|P_{m} f\right\|_{L_{x}^{2}}\right) \\
\lesssim & \sum_{m} 2^{-|k-m|}\left(\Delta_{0}\left\|P_{m} f(t)\right\|_{L_{t}^{\infty} L_{x}^{2}}+\int_{0}^{t}\left\|P_{m} f\right\|_{L_{x}^{2}}\right)
\end{aligned}
$$


Hence,

$$
\sum_{k \geq 0}\left\|\int_{0}^{t}\left[\nabla_{L}, P_{k}\right]\right\|_{L_{t}^{\infty} L_{x}^{2}} \lesssim \sum_{m}\left(\Delta_{0} \cdot\left\|P_{m} f(t)\right\|_{L_{t}^{\infty} L_{x}^{2}}+\int_{0}^{t}\left\|P_{m} f\right\|_{L_{x}^{2}}\right)
$$

as desired.

Remark 11.5. In the argument above we have used the following simple bounds for $\tilde{m}(\tau)=\tau^{\frac{\epsilon}{2}} m(\tau)$ and $\hat{m}(\tau)=\tau^{-\frac{\epsilon}{2}} m(\tau)$ :

$$
\int_{0}^{\infty} \tilde{m}(\tau), \quad \int_{0}^{\infty} \hat{m}(\tau) \lesssim 1, \quad \int_{0}^{a} \tilde{m}(\tau) \lesssim a, \quad \int_{A}^{\infty} \hat{m}(\tau) \lesssim A^{-1}
$$

which hold for all sufficiently small $a$ and all sufficiently large A. Proposition 11.2 now easily follows.

Proof of Lemma 11.4: We prove lemma 11.4 by a bootstrap argument. More precisely we assume that for any $\tau \approx 2^{-2 m_{0}}$ and any $g$,

$$
\|\mathcal{L}(\tau) g\|_{L_{t}^{\infty} L_{x}^{2}} \lesssim M \sum_{k \geq 0} 2^{-\epsilon\left|m_{0}-k\right|}\left\|P_{k} g\right\|_{L_{t}^{\infty} L_{x}^{2}}
$$

with some positive sufficiently large constant $M$ and a fixed positive constant $\epsilon>0$, both independent on the function ${ }^{22} \mathrm{~g}$. Also, since the estimate above holds for all positive values of $m_{0}$ we deduce,

$$
\sup _{0 \leq \tau \leq 1}\|\mathcal{L}(\tau) g\|_{L_{t}^{\infty} L_{x}^{2}} \lesssim M\|g\|_{\mathcal{B}^{0}}
$$

We shall show that the bounds (114) and (115) implies the stronger estimate

$$
\|\mathcal{L}(\tau) g\|_{L_{t}^{\infty} L_{x}^{2}} \lesssim \sum_{k \geq 0} 2^{-\epsilon\left|m_{0}-k\right|}\left(\Delta_{0} M \cdot\left\|P_{k} g\right\|_{L_{t}^{2} L_{x}^{2}}+\int_{0}^{t}\left\|P_{k} g\right\|_{L_{t}^{2} L_{x}^{2}}\right)
$$

from which the desired estimates (113), 112 follow.

We shall often use the following heat flow estimate similar to (109)

$$
\left\|P_{k} U(\tau) f\right\|_{L_{x}^{2}} \lesssim\left(1+2^{2 k} \tau\right)^{-2}\left\|P_{k} f\right\|_{L_{x}^{2}}
$$

We derive

$$
\left[\nabla_{L}, U(\tau)\right] f=\int_{0}^{\tau} U\left(\tau-\tau^{\prime}\right)\left[\nabla_{L}, \Delta\right] U\left(\tau^{\prime}\right) f d s
$$

Recall, see proposition 3.12, the following commutator formula for scalars,

$$
\begin{aligned}
{\left[\nabla_{L}, \Delta\right] f } & =-\operatorname{tr} \chi \Delta f+A \cdot \nabla^{2} f+\nabla \operatorname{tr} \chi \cdot \nabla f+\left(A+\frac{1}{r}\right) \cdot A \cdot \nabla f \\
& =-\frac{2}{r} \Delta f+A \cdot \nabla^{2} f+\nabla \operatorname{tr} \chi \cdot \nabla f+\left(A+\frac{1}{r}\right) \cdot A \cdot \nabla f
\end{aligned}
$$

\footnotetext{
${ }^{22}$ Clearly this estimate holds true with a constant $M$ which might depend on more derivatives of the background metric.
} 
Therefore,

$$
\begin{aligned}
\mathcal{L}(\tau) f & =I_{1}+I_{2}+I_{3}+I_{4} \\
I_{1} & =\int_{0}^{t} d s \int_{0}^{\tau} d \tau U\left(\tau-\tau^{\prime}\right)\left(A \cdot \nabla^{2} U\left(\tau^{\prime}\right) f\right) \\
I_{2} & =\int_{0}^{t} d s \int_{0}^{\tau} d \tau U\left(\tau-\tau^{\prime}\right)\left(\nabla A \cdot \nabla U\left(\tau^{\prime}\right) f\right) \\
I_{3} & =-\frac{2}{r} \int_{0}^{t} d s \int_{0}^{\tau} d \tau U\left(\tau-\tau^{\prime}\right) \Delta U\left(\tau^{\prime}\right) f \\
I_{4} & =\int_{0}^{t} d s \int_{0}^{\tau} d \tau U\left(\tau-\tau^{\prime}\right)\left(\left(A+\frac{1}{r}\right) \cdot A \cdot \nabla U\left(\tau^{\prime}\right) f\right)
\end{aligned}
$$

Estimate for $I_{2}$. Using the dual strong scalar Bernstein inequality for $U\left(\tau-\tau^{\prime}\right)$ followed by Hölder and the heat flow estimate (117), we infer

$$
\begin{aligned}
\left\|I_{2}(\tau)\right\|_{L_{t}^{\infty} L_{x}^{2}} & \lesssim \int_{0}^{\tau}\left(\tau-\tau^{\prime}\right)^{-\frac{1}{2}}\left\|\nabla A \cdot \nabla U\left(\tau^{\prime}\right) f\right\|_{L_{t}^{1} L_{x}^{1}} \\
& \lesssim\|\nabla A\|_{L_{t}^{2} L_{x}^{2}} \cdot \int_{0}^{\tau}\left(\tau-\tau^{\prime}\right)^{-\frac{1}{2}}\left\|\nabla U\left(\tau^{\prime}\right) f\right\|_{L_{t}^{2} L_{x}^{2}} \\
& \lesssim \Delta_{0} \sum_{m} \int_{0}^{\tau}\left(\tau-\tau^{\prime}\right)^{-\frac{1}{2}}\left\|\nabla P_{m} U\left(\tau^{\prime}\right) f\right\|_{L_{t}^{2} L_{x}^{2}} \\
& \lesssim \Delta_{0} \sum_{m} 2^{m}\left\|P_{m} f\right\|_{L_{t}^{2} L_{x}^{2}} \int_{0}^{\tau}\left(1+2^{2 m} \tau^{\prime}\right)^{-2}\left(\tau-\tau^{\prime}\right)^{-\frac{1}{2}} d \tau^{\prime}
\end{aligned}
$$

Hence,

$$
\left\|I_{2}(\tau)\right\|_{L_{t}^{\infty} L_{x}^{2}} \lesssim \Delta_{0} \sum_{m} 2^{-\left|m-m_{0}\right|}\left\|P_{m} f\right\|_{L_{t}^{2} L_{x}^{2}} \quad \text { for } \quad \tau \approx 2^{-2 m_{0}}
$$

The last statement follows from the following:

Lemma 11.6. For $\tau \approx 2^{-2 m_{0}}$ we have,

$$
J_{m}=2^{m} \int_{0}^{\tau}\left(1+2^{2 m} \tau^{\prime}\right)^{-2}\left(\tau-\tau^{\prime}\right)^{-\frac{1}{2}} d \tau^{\prime} \lesssim 2^{-\left|m-m_{0}\right|}
$$

Proof: Indeed for $\tau \approx 2^{-2 m_{0}} \leq 2^{-2 m}$

$$
J_{m} \lesssim 2^{m} \int_{0}^{\tau}\left(\tau-\tau^{\prime}\right)^{-\frac{1}{2}} d \tau^{\prime} \lesssim 2^{m} \tau^{\frac{1}{2}} \lesssim 2^{m-m_{0}}=2^{-\left|m-m_{0}\right|}
$$

For $\tau \approx 2^{-2 m_{0}} \geq 2^{-2 m}$,

$$
\begin{aligned}
J_{m} & \lesssim 2^{m} \int_{0}^{\tau / 2}\left(1+2^{2 m} \tau^{\prime}\right)^{-2}\left(\tau-\tau^{\prime}\right)^{-\frac{1}{2}} d \tau^{\prime}+2^{m} \int_{\tau / 2}^{\tau}\left(1+2^{2 m} \tau^{\prime}\right)^{-2}\left(\tau-\tau^{\prime}\right)^{-\frac{1}{2}} d \tau^{\prime} \\
& \lesssim 2^{m} \tau^{-1 / 2} \int_{0}^{\tau / 2}\left(1+2^{2 m} \tau^{\prime}\right)^{-2} d \tau^{\prime}+2^{m}\left(1+2^{2 m} \tau\right)^{-2} \tau^{1 / 2} \lesssim 2^{-m} \tau^{-1 / 2} \lesssim 2^{m_{0}-m} \\
& =2^{-\left|m-m_{0}\right|}
\end{aligned}
$$


Estimate for $I_{3}$. We rewrite $I_{3}$ as follows ${ }^{23}$,

$$
\begin{aligned}
I_{3} & =\int_{0}^{t} d s \int_{0}^{\tau} d \tau^{\prime} U\left(\tau-\tau^{\prime}\right) \Delta U\left(\tau^{\prime}\right) f=\int_{0}^{t} d s \int_{0}^{\tau} d \tau^{\prime} U\left(\tau-\tau^{\prime}\right) U\left(\tau^{\prime}\right) \Delta f \\
& =\tau \int_{0}^{t} d s \Delta U(\tau) f
\end{aligned}
$$

Hence, using (117) and $\tau \approx 2^{-2 m_{0}}$,

$$
\begin{aligned}
\left\|I_{3}(\tau)\right\|_{L_{t}^{\infty} L_{x}^{2}} & \lesssim \tau\|\Delta U(\tau) f\|_{L_{t}^{1} L_{x}^{2}} \lesssim \tau \sum_{m}\left\|\Delta U(\tau) P_{m} f\right\|_{L_{t}^{1} L_{x}^{2}} \\
& \lesssim \sum_{m} \tau \cdot 2^{2 m}\left(1+\tau \cdot 2^{2 m}\right)^{-2}\left\|P_{m} f\right\|_{L_{t}^{1} L_{x}^{2}} \\
& \lesssim \sum_{m} 2^{2\left(m-m_{0}\right)}\left(1+2^{2\left(m-m_{0}\right)}\right)^{-2}\left\|P_{m} f\right\|_{L_{t}^{1} L_{x}^{2}} \\
& \lesssim \sum_{m} 2^{-\left|m-m_{0}\right|}\left\|P_{m} f\right\|_{L_{t}^{1} L_{x}^{2}} .
\end{aligned}
$$

Estimates for $I_{4}$. We proceed precisely as for $I_{2}$, by noticing that $\|A\|_{L_{t}^{4} L_{x}^{4}} \lesssim \Delta_{0}$ in view of (29) and lemma 3.10,

$$
\begin{aligned}
\left\|I_{4}(\tau)\right\|_{L_{t}^{\infty} L_{x}^{2}} & \lesssim \int_{0}^{\tau}\left(\tau-\tau^{\prime}\right)^{-\frac{1}{2}}\left\|\left(A+\frac{1}{r}\right) \cdot A \cdot \nabla U\left(\tau^{\prime}\right) f\right\|_{L_{t}^{1} L_{x}^{1}} \\
& \lesssim\left\|\left(A+\frac{1}{r}\right) \cdot A\right\|_{L_{t}^{2} L_{x}^{2}} \cdot \int_{0}^{\tau}\left(\tau-\tau^{\prime}\right)^{-\frac{1}{2}}\left\|\nabla U\left(\tau^{\prime}\right) f\right\|_{L_{t}^{2} L_{x}^{2}} \\
& \lesssim \Delta_{0} \sum_{m} \int_{0}^{\tau}\left(\tau-\tau^{\prime}\right)^{-\frac{1}{2}}\left\|\nabla P_{m} U\left(\tau^{\prime}\right) f\right\|_{L_{t}^{2} L_{x}^{2}} \\
& \lesssim \Delta_{0} \sum_{m} 2^{m}\left\|P_{m} f\right\|_{L_{t}^{2} L_{x}^{2}} \int_{0}^{\tau}\left(1+2^{2 m} \tau^{\prime}\right)^{-2}\left(\tau-\tau^{\prime}\right)^{-\frac{1}{2}} d \tau^{\prime}(121) \\
& \lesssim \Delta_{0} \cdot \sum_{m} 2^{-\left|m-m_{0}\right|}\left\|P_{m} f\right\|_{L_{t}^{1} L_{x}^{2}}
\end{aligned}
$$

for $\tau \approx 2^{-2 m_{0}}$.

Estimates for $I_{1}$ This is the most delicate term; indeed it is because of this term that we need to make the bootstrap assumption (114). The difficulty stems from the fact that we need to use the trace norm assumption $\|A\|_{L_{x}^{\infty} L_{t}^{2}} \lesssim \Delta_{0}$ which means we have to bring the integration $\int_{0}^{t}$, along null geodesics, in front of $U\left(\tau-\tau^{\prime}\right)$ in the formula for $I_{1}$, see (118). This brings in the commutator between $\int_{0}^{t}$ and $U\left(\tau-\tau^{\prime}\right)$ which we shall treat according to the formula:

$$
\left[\int_{0}^{t}, U\left(\tau-\tau^{\prime}\right)\right] g=\int_{0}^{t}\left[\nabla_{L}, U\left(\tau-\tau^{\prime}\right)\right] \int_{0}^{t^{\prime}} g=\mathcal{L}\left(\tau-\tau^{\prime}\right) \int_{0}^{t} g .
$$

\footnotetext{
${ }^{23}$ We neglect the factor $-\frac{2}{r}$ which plays no role in the estimates.
} 
Thus,

$$
\begin{aligned}
I_{1} & =\int_{0}^{\tau} U\left(\tau-\tau^{\prime}\right)\left(\int_{0}^{t} A \cdot \nabla^{2} U\left(\tau^{\prime}\right) f\right) d \tau^{\prime}+\int_{0}^{\tau} \mathcal{L}\left(\tau-\tau^{\prime}\right)\left(\int_{0}^{t} A \cdot \nabla^{2} U\left(\tau^{\prime}\right) f\right) d \tau^{\prime} \\
& =I_{11}+I_{12}
\end{aligned}
$$

Using Lemma 3.13 and the dual strong scalar Bernstein inequality, we estimate

$$
\begin{aligned}
\left\|I_{11}(\tau)\right\|_{L_{t}^{\infty} L_{x}^{2}} & \lesssim \int_{0}^{\tau}\left\|U\left(\tau-\tau^{\prime}\right) \int_{0}^{t} \operatorname{div}\left(A \cdot \nabla U\left(\tau^{\prime}\right) f\right)\right\|_{L_{x}^{2}} \\
& +\int_{0}^{\tau}\left\|U\left(\tau-\tau^{\prime}\right) \int_{0}^{t} \nabla A \cdot \nabla U\left(\tau^{\prime}\right) f\right\|_{L_{x}^{2}} \\
& \lesssim \int_{0}^{\tau}\left(\tau-\tau^{\prime}\right)^{-\frac{1}{2}} d \tau^{\prime}\left(\left\|A \cdot \nabla U\left(\tau^{\prime}\right) f\right\|_{L_{x}^{2} L_{t}^{1}}+\left\|\nabla A \cdot \nabla U\left(\tau^{\prime}\right) f\right\|_{L_{x}^{1} L_{t}^{1}}\right) \\
& \lesssim\left(\|A\|_{L_{x}^{\infty} L_{t}^{2}}+\|\nabla A\|_{L_{t}^{2} L_{x}^{2}}\right) \int_{0}^{\tau}\left(\tau-\tau^{\prime}\right)^{-\frac{1}{2}}\left\|\nabla U\left(\tau^{\prime}\right) f\right\|_{L_{t}^{2} L_{x}^{2}} \\
& \lesssim \Delta_{0} \int_{0}^{\tau}\left(\tau-\tau^{\prime}\right)^{-\frac{1}{2}}\left\|\nabla U\left(\tau^{\prime}\right) f\right\|_{L_{t}^{2} L_{x}^{2}}
\end{aligned}
$$

Thus, continuing exactly as for $I_{2}$,

$$
\left\|I_{11}(\tau)\right\|_{L_{t}^{\infty} L_{x}^{2}} \lesssim \Delta_{0} \sum_{m} 2^{-\left|m-m_{0}\right|}\left\|P_{m} f\right\|_{L_{t}^{2} L_{x}^{2}}, \quad \text { for } \quad \tau \approx 2^{-2 m_{0}}
$$

We now estimate $I_{12}$ with the help of the bootstrap assumption (114),(115) According to these we have, for any smooth function $g$, the following bounds,

$$
\begin{aligned}
& \left\|\mathcal{L}\left(\tau^{\prime}\right) g\right\|_{L_{t}^{\infty} L_{x}^{2}} \leq 2 M \sum_{m} 2^{-\epsilon\left|m-m_{0}\right|}\left\|P_{m} g\right\|_{L_{t}^{\infty} L_{x}^{2}}, \quad \tau^{\prime} \in\left[\frac{\tau}{2}, \tau\right], \quad \tau \approx 2^{-2 m_{0}} \\
& \sup _{0 \leq \tau^{\prime} \leq 1}\left\|\mathcal{L}\left(\tau^{\prime}\right) g\right\|_{L_{t}^{\infty} L_{x}^{2}} \leq M\|g\|_{B^{0}},
\end{aligned}
$$

Therefore,

$$
\begin{aligned}
\left\|I_{12}\right\|_{L_{t}^{\infty} L_{x}^{2}} & \lesssim\left\|\int_{0}^{\tau} \mathcal{L}\left(\tau-\tau^{\prime}\right)\left(\int_{0}^{t} A \cdot \nabla^{2} U\left(\tau^{\prime}\right) f\right) d s\right\|_{L_{t}^{\infty} L_{x}^{2}} \\
& \lesssim \int_{0}^{\frac{\tau}{2}}\left\|\mathcal{L}\left(\tau-\tau^{\prime}\right)\left(\int_{0}^{t} A \cdot \nabla^{2} U\left(\tau^{\prime}\right) f\right) d s\right\|_{L_{t}^{\infty} L_{x}^{2}} \\
& +\int_{\frac{\tau}{2}}^{\tau}\left\|\mathcal{L}\left(\tau-\tau^{\prime}\right)\left(\int_{0}^{t} A \cdot \nabla^{2} U\left(\tau^{\prime}\right) f\right) d s\right\|_{L_{t}^{\infty} L_{x}^{2}} \\
& \lesssim 2 M \int_{0}^{\frac{\tau}{2}} \sum_{m} 2^{-\epsilon\left|m-m_{0}\right|}\left\|P_{m} \int_{0}^{t} A \cdot \nabla^{2} U\left(\tau^{\prime}\right) f\right\|_{L_{t}^{\infty} L_{x}^{2}} \\
& +M \int_{\frac{\tau}{2}}^{\tau}\left\|\int_{0}^{t} A \cdot \nabla^{2} U\left(\tau^{\prime}\right) f\right\|_{\mathcal{B}^{0}}=\mathcal{I}_{1}+\mathcal{I}_{2}
\end{aligned}
$$


Using the product Besov estimate (79) and the estimates (110), (111) we obtain that

$$
\begin{aligned}
\int_{\frac{\tau}{2}}^{\tau}\left\|\int_{0}^{t} A \cdot \nabla^{2} U\left(\tau^{\prime}\right) f\right\|_{\mathcal{B}^{0}} d \tau^{\prime} & \lesssim \Delta_{0} \int_{\frac{\tau}{2}}^{\tau}\left\|\nabla^{2} U\left(\tau^{\prime}\right) f\right\|_{\mathcal{P}^{0}} d \tau^{\prime} \\
& \lesssim \Delta_{0} \tau \sum_{k \geq 0} \min \left(2^{2 k}, 2^{-2 k} \tau^{-2}\right)\left\|P_{k} f\right\|_{L_{t}^{2} L_{x}^{2}} \\
& \lesssim \Delta_{0} \sum_{k \geq 0} 2^{-2\left|k-m_{0}\right|}\left\|P_{k} f\right\|_{L_{t}^{2} L_{x}^{2}}
\end{aligned}
$$

We conclude that

$$
\mathcal{I}_{2} \lesssim \Delta_{0} M \sum_{k \geq 0} 2^{-2\left|k-m_{0}\right|}\left\|P_{k} f\right\|_{L_{t}^{2} L_{x}^{2}}
$$

To estimate $\mathcal{I}_{1}$ we observe that according to the estimate (81) of the remark 4.10

$$
\begin{aligned}
\left\|P_{m} \int_{0}^{t} A \cdot \nabla^{2} U\left(\tau^{\prime}\right) f\right\|_{L_{t}^{\infty} L_{x}^{2}} & \lesssim\left(\mathcal{N}_{1}[A]+\|A\|_{L_{x}^{\infty} L_{t}^{2}}\right) \cdot \sum_{k \geq 0} 2^{-\sigma|m-k|}\left\|P_{k} \nabla^{2} U\left(\tau^{\prime}\right) f\right\|_{L_{t}^{2} L_{x}^{2}} \\
& \lesssim \Delta_{0} \sum_{k \geq 0} 2^{-\sigma|m-k|}\left\|P_{k} \nabla^{2} U\left(\tau^{\prime}\right) f\right\|_{L_{t}^{2} L_{x}^{2}}
\end{aligned}
$$

for some positive constant $\sigma>\epsilon$. Therefore, with the help of lemma 11.7 below,

$$
\begin{aligned}
\mathcal{I}_{1} & \lesssim 2 \Delta_{0} M \sum_{m} 2^{-\epsilon\left|m-m_{0}\right|} \sum_{k \geq 0} 2^{-\sigma|m-k|} \int_{0}^{\frac{\tau}{2}}\left\|P_{k} \nabla^{2} U\left(\tau^{\prime}\right) f\right\|_{L_{t}^{2} L_{x}^{2}} \\
& \lesssim \Delta_{0} M \cdot \sum_{m} 2^{-\epsilon\left|m-m_{0}\right|} \int_{0}^{\frac{\tau}{2}}\left\|P_{m} \nabla^{2} U\left(\tau^{\prime}\right) f\right\|_{L_{t}^{2} L_{x}^{2}}=\Delta_{0} M \cdot \mathcal{J} \\
& \lesssim \Delta_{0} M \cdot \sum_{k \geq 0} 2^{-\epsilon\left|k-m_{0}\right|}\left\|P_{k} f\right\|_{L_{t}^{2} L_{x}^{2}}
\end{aligned}
$$

Thus,

$$
\left\|I_{12}(\tau)\right\|_{L_{t}^{\infty} L_{x}^{2}} \lesssim M \Delta_{0} \sum_{m} 2^{-\left|m-m_{0}\right|}\left\|P_{m} f\right\|_{L_{t}^{2} L_{x}^{2}}, \quad \text { for } \quad \tau \approx 2^{-2 m_{0}},
$$

which ends the proof of the improved estimate (116) and therefore also of lemma 11.4 .

It only remains to prove the following,

Lemma 11.7. For $\tau \approx 2^{-2 m_{0}}$ the integral $\mathcal{J}=\mathcal{J}^{+}+\mathcal{J}^{-}$defined by

$$
\begin{aligned}
& \mathcal{J}^{+}=\sum_{m \geq m_{0}} 2^{-\epsilon\left|m-m_{0}\right|} \int_{0}^{\frac{\tau}{2}}\left\|P_{m} \nabla^{2} U\left(\tau^{\prime}\right) f\right\|_{L_{t}^{2} L_{x}^{2}} \\
& \mathcal{J}^{-}=\sum_{0 \leq m<m_{0}} 2^{-\epsilon\left|m-m_{0}\right|} \int_{0}^{\frac{\tau}{2}}\left\|P_{m} \nabla^{2} U\left(\tau^{\prime}\right) f\right\|_{L_{t}^{2} L_{x}^{2}}
\end{aligned}
$$

verifies the estimate,

$$
\mathcal{J} \lesssim \sum_{k \geq 0} 2^{-\epsilon\left|k-m_{0}\right|}\left\|P_{k} f\right\|_{L_{t}^{2} L_{x}^{2}}
$$




\section{Proof :}

1) Estimates for $\mathcal{J}_{+}$. We are in the case $m \geq m_{0}\left(\right.$ or $\left.2^{-2 m} \leq \tau\right)$ We decompose further

$$
\begin{aligned}
\left\|P_{m} \nabla^{2} U\left(\tau^{\prime}\right) f\right\|_{L_{t}^{2} L_{x}^{2}} & \lesssim \sum_{k \geq 0}\left\|P_{m} \nabla^{2} P_{k} U\left(\tau^{\prime}\right) f\right\|_{L_{t}^{2} L_{x}^{2}} \\
& \lesssim \sum_{k \leq m_{0}}\left\|P_{m} \nabla^{2} P_{k} U\left(\tau^{\prime}\right) f\right\|_{L_{t}^{2} L_{x}^{2}}+\sum_{k \geq m}\left\|P_{m} \nabla^{2} P_{k} U\left(\tau^{\prime}\right) f\right\|_{L_{t}^{2} L_{x}^{2}} \\
& +\sum_{m_{0}<k<m}\left\|P_{m} \nabla^{2} P_{k} U\left(\tau^{\prime}\right) f\right\|_{L_{t}^{2} L_{x}^{2}}=J_{1}+J_{2}+J_{3}
\end{aligned}
$$

Let $\mathcal{J}_{1}^{+}, \mathcal{J}_{2}^{+}, \mathcal{J}_{3}^{+}$be the corresponding contributions to $\mathcal{J}$. The term $\mathcal{J}_{3}$ is easiest to estimate,

$$
J_{3}=\sum_{m_{0}<k<m}\left\|P_{m} \nabla^{2} P_{k} U\left(\tau^{\prime}\right) f\right\|_{L_{t}^{2} L_{x}^{2}} \lesssim \sum_{m_{0}<k<m}\left\|\nabla^{2} P_{k} U\left(\tau^{\prime}\right) f\right\|_{L_{t}^{2} L_{x}^{2}}
$$

Using lemma 11.3 we obtain

$$
\int_{0}^{\frac{\tau}{2}} J_{3} \lesssim \sum_{m_{0}<k<m} \int_{0}^{\frac{\tau}{2}}\left\|\Delta U\left(\tau^{\prime}\right) P_{k} f\right\|_{L_{t}^{2} L_{x}^{2}} \lesssim \sum_{m_{0}<k<m}\left\|P_{k} f\right\|_{L_{t}^{2} L_{x}^{2}}
$$

Therefore,

$$
\mathcal{J}_{3}^{+}=\sum_{m>m_{0}} 2^{-\epsilon\left|m-m_{0}\right|} \sum_{m_{0}<k<m}\left\|P_{k} f\right\|_{L_{t}^{2} L_{x}^{2}} \lesssim \sum_{k \geq 0} 2^{-\epsilon\left|k-m_{0}\right|}\left\|P_{k} f\right\|_{L_{t}^{2} L_{x}^{2}}
$$

To estimate $\mathcal{J}_{1}^{+}$we proceed as follows. Applying the dual finite band property followed by the finite band property of the $P_{k}^{\prime} s$, we obtain

$$
\begin{aligned}
J_{1} \lesssim 2^{m} \sum_{k \geq m}\left\|\nabla P_{k} U\left(\tau^{\prime}\right) f\right\|_{L_{t}^{2} L_{x}^{2}} & \lesssim \sum_{m \geq k} 2^{m+k}\left\|P_{k} U\left(\tau^{\prime}\right) f\right\|_{L_{t}^{2} L_{x}^{2}} \\
& \lesssim \sum_{k \geq m} 2^{m-k}\left\|\Delta U\left(\tau^{\prime}\right) P_{k} f\right\|_{L_{t}^{2} L_{x}^{2}}
\end{aligned}
$$

Once again, lemma 11.3 gives

$$
\int_{0}^{\frac{\tau}{2}} J_{1} \lesssim \sum_{k \geq m} 2^{m-k}\left\|P_{k} f\right\|_{L_{t}^{2} L_{x}^{2}}
$$

Thus,

$$
\mathcal{J}^{+}=\sum_{m>m_{0}} 2^{-\epsilon\left|m-m_{0}\right|} \sum_{k \geq m} 2^{-|m-k|}\left\|P_{k} f\right\|_{L_{t}^{2} L_{x}^{2}} \lesssim \Delta_{0} M \sum_{k \geq 0} 2^{-\epsilon\left|k-m_{0}\right|}\left\|P_{k} f\right\|_{L_{t}^{2} L_{x}^{2}} .
$$

Finally, we have for $J_{2}$.

$$
J_{2} \lesssim \sum_{k<m_{0}}\left\|\nabla^{2} P_{k} U\left(\tau^{\prime}\right) f\right\|_{L_{t}^{2} L_{x}^{2}} \lesssim \sum_{k<m_{0}} 2^{2 k}\left\|P_{k} f\right\|_{L_{t}^{2} L_{x}^{2}}
$$

Therefore,

$$
\int_{0}^{\frac{\tau}{2}} J_{2} \lesssim \sum_{k<m_{0}} 2^{2 k} \tau\left\|P_{k} f\right\|_{L_{t}^{2} L_{x}^{2}} \lesssim \sum_{k<m_{0}} 2^{-2\left|k-m_{0}\right|}\left\|P_{k} f\right\|_{L_{t}^{2} L_{x}^{2}} .
$$


and we infer that

$$
\mathcal{J}_{2}^{+}=\sum_{m>m_{0}} 2^{-\epsilon\left|m-m_{0}\right|} \sum_{k<m_{0}} 2^{-2\left|m_{0}-k\right|}\left\|P_{k} f\right\|_{L_{t}^{2} L_{x}^{2}} \lesssim \sum_{k \geq 0} 2^{-\epsilon\left|k-m_{0}\right|}\left\|P_{k} f\right\|_{L_{t}^{2} L_{x}^{2}} .
$$

2) Estimate for $\mathcal{J}^{-}$. We are in the case $m \leq m_{0}\left(2^{-2 m} \geq \tau\right)$ As before we decompose further as follows:

$$
\begin{aligned}
\left\|P_{m} \nabla^{2} U\left(\tau^{\prime}\right) f\right\|_{L_{t}^{2} L_{x}^{2}} & \lesssim \sum_{k \geq 0}\left\|P_{m} \nabla^{2} P_{k} U\left(\tau^{\prime}\right) f\right\|_{L_{t}^{2} L_{x}^{2}} \\
& \lesssim \sum_{k \leq m}\left\|P_{m} \nabla^{2} P_{k} U\left(\tau^{\prime}\right) f\right\|_{L_{t}^{2} L_{x}^{2}}+\sum_{k \geq m_{0}}\left\|P_{m} \nabla^{2} P_{k} U\left(\tau^{\prime}\right) f\right\|_{L_{t}^{2} L_{x}^{2}} \\
& +\sum_{m<k<m_{0}}\left\|P_{m} \nabla^{2} P_{k} U\left(\tau^{\prime}\right) f\right\|_{L_{t}^{2} L_{x}^{2}}=N_{1}+N_{2}+N_{3}
\end{aligned}
$$

and denote by $\mathcal{J}_{1}^{-}, \mathcal{J}_{2}^{-}, \mathcal{J}_{3}^{-}$the corresponding contributions to $\mathcal{J}^{-}$. The estimates for $N_{3}$ and $\mathcal{J}_{3}^{-}$are identical to that for $J_{3}, \mathcal{J}_{3}^{+}$.

To estimate $N_{1}$ we proceed as follows.

$$
N_{1} \lesssim \sum_{k \leq m}\left\|\nabla^{2} P_{k} U\left(\tau^{\prime}\right) f\right\|_{L_{t}^{2} L_{x}^{2}} \lesssim \sum_{k \leq m} 2^{2 k}\left\|P_{k} f\right\|_{L_{t}^{2} L_{x}^{2}}
$$

Integrating in $s$ and using that $\tau \approx 2^{-2 m_{0}}$ we obtain the bound

$$
\mathcal{J}_{1}^{-}=\sum_{m \leq m_{0}} 2^{-\epsilon\left|m-m_{0}\right|} \sum_{k \leq m} 2^{-2\left|m_{0}-k\right|}\left\|P_{k} f\right\|_{L_{t}^{2} L_{x}^{2}} \lesssim \sum_{k \geq 0} 2^{-2\left|k-m_{0}\right|}\left\|P_{k} f\right\|_{L_{t}^{2} L_{x}^{2}}
$$

Finally, we have for $N_{2}$.

$$
N_{2} \lesssim \sum_{k \geq m_{0}}\left\|P_{m} \nabla^{2} P_{k} U\left(\tau^{\prime}\right) f\right\|_{L_{t}^{2} L_{x}^{2}} \lesssim \sum_{k \geq m_{0}} 2^{m-k}\left\|\Delta U\left(\tau^{\prime}\right) P_{k} f\right\|_{L_{t}^{2} L_{x}^{2}}
$$

Integrating in $s$, using lemma 11.3, we obtain the estimate

$$
\mathcal{J}_{2}^{-}=\sum_{m \leq m_{0}} 2^{-\epsilon\left|m-m_{0}\right|} \sum_{k \geq m_{0}} 2^{-|m-k|}\left\|P_{k} f\right\|_{L_{t}^{2} L_{x}^{2}} \lesssim \sum_{k \geq 0} 2^{-\epsilon\left|k-m_{0}\right|}\left\|P_{k} f\right\|_{L_{t}^{2} L_{x}^{2}} .
$$

Combining the estimates involving $\mathcal{J}_{1}^{+}, \mathcal{J}_{2}^{+}, \mathcal{J}_{3}^{+}$and $\mathcal{J}_{1}^{-}, \mathcal{J}_{2}^{-}, \mathcal{J}_{3}$ we conclude that

$$
\mathcal{J} \lesssim \sum_{k \geq 0} 2^{-\epsilon\left|k-m_{0}\right|}\left\|P_{k} f\right\|_{L_{t}^{2} L_{x}^{2}}
$$

as desired.

\section{Commutator estimates}

This section we prove the commutator lemma 5.3 which we recall below.

Proposition 12.1. For any smooth $S$-tangent tensor field $F$ and an arbitrary $1 \leq q<2$ the following estimate holds true

$$
\left\|\left[P_{k}, \nabla_{L}\right] f\right\|_{L_{t}^{q} L_{x}^{2}}+2^{-k}\left\|\nabla\left[P_{k}, \nabla_{L}\right] f\right\|_{L_{t}^{q} L_{x}^{2}} \lesssim 2^{-\frac{k}{2}+} \mathcal{N}_{1}[F]
$$


In addition,

$$
\left\|\left[P_{k}, \nabla_{L}\right] f\right\|_{L_{t}^{1} L_{x}^{2}}+2^{-k}\left\|\nabla\left[P_{k}, \nabla_{L}\right] f\right\|_{L_{t}^{1} L_{x}^{2}} \lesssim 2^{-k+} \mathcal{N}_{1}[F]
$$

Proof : Using the definition of the LP projection $P_{k}$ we obtain

$$
\begin{aligned}
{\left[P_{k}, \nabla_{L}\right] F } & =\int_{0}^{\infty} m_{k}(\tau)\left[U(\tau), \nabla_{L}\right] F d \tau \\
\Phi(\tau) & =\left[U(\tau), \nabla_{L}\right] F
\end{aligned}
$$

To calculate $\left[U(\tau), \nabla_{L}\right] F$ we recall the commutator formula for $\left[\nabla_{L}, \Delta\right]$ from proposition 3.12

$$
\begin{aligned}
{\left[\nabla_{L}, \Delta\right] F } & =-\operatorname{tr} \chi \Delta F+A \cdot \nabla^{2} F+\nabla A \cdot \nabla F+\left(A+\frac{1}{r}\right) \cdot A \cdot \nabla F \\
& +\beta \cdot \nabla F+\nabla\left(\beta \cdot F+\left(A+\frac{1}{r}\right) \cdot A \cdot F\right)
\end{aligned}
$$

which we rewrite in the simplified, symbolic, form,

$$
\begin{aligned}
{\left[\nabla_{L}, \Delta\right] F } & =\nabla\left(\left(\frac{1}{r}+A\right) \cdot \nabla F+\left(\beta+\left(A+\frac{1}{r}\right) \cdot A\right) \cdot F\right) \\
& +\left(\nabla A+\left(\beta+\left(A+\frac{1}{r}\right) \cdot A\right)\right) \cdot \nabla F
\end{aligned}
$$

Observe that the terms $\left(A+\frac{1}{r}\right) \cdot A$ and $\nabla A$ have same or better estimates than $\beta$. Therefore we can discard them and simplify,

$$
\left[\nabla_{L}, \Delta\right] F=\nabla\left(\left(\frac{1}{r}+A\right) \cdot \nabla F+\beta \cdot F\right)+\beta \cdot \nabla F
$$

Consequently, setting $\hat{A}=A+\frac{1}{r}$,

$$
\begin{aligned}
\Phi(\tau) & =\int_{0}^{\tau} U\left(\tau-\tau^{\prime}\right)\left[\Delta, \nabla_{L}\right] U\left(\tau^{\prime}\right) F \\
& =\int_{0}^{\tau} U\left(\tau-\tau^{\prime}\right) \nabla\left(\hat{A} \cdot \nabla U\left(\tau^{\prime}\right) F+\beta U\left(\tau^{\prime}\right) F\right) \\
& +\int_{0}^{\tau} U\left(\tau-\tau^{\prime}\right) \beta \cdot \nabla U\left(\tau^{\prime}\right) F \\
& =\Phi_{1}(\tau)+\Phi_{2}(\tau)
\end{aligned}
$$

In what follows we shall rely on the following heat flow estimates:

Lemma 12.2. For any $2 \leq p<\infty$,

$$
\begin{aligned}
\|U(\tau) G\|_{L_{x}^{\infty}} & \lesssim\left(1+\|K\|_{L_{x}^{2}}^{\frac{1}{p-1}}\right)\left\|U\left(\tau^{\prime} / 2\right) G\right\|_{H_{x}^{1}}, \\
\|U(\tau) G\|_{L_{x}^{\infty}} & \lesssim\left\langle\tau^{-\frac{1}{r}}\right\rangle\left(1+\|K\|_{L_{x}^{2}}^{\frac{2}{r(p-1)}}\right)\|G\|_{L_{x}^{r}}, \quad 2 \leq \forall r \leq \infty \\
\left\|\nabla^{2} U(\tau) G\right\|_{L_{x}^{2}} & \lesssim\left(\tau^{-\frac{1}{2}}+\|K\|_{L_{x}^{2}}^{\frac{p}{p-1}}\right)\left\|U\left(\tau^{\prime} / 2\right) G\right\|_{H_{x}^{1}} .
\end{aligned}
$$


Proof: The estimates follow easily, by interpolation, from the heat flow estimates of proposition 3.17combined with the Bochner and $L^{\infty}$ inequalities (41), (40).

Our proof will now proceed as follows:

Step 1: We shall first establish the following estimates for $\Phi_{1}$ :

$$
\begin{aligned}
\left\|\Phi_{1}(\tau)\right\|_{L_{t}^{q} L_{x}^{2}} & \lesssim \max \left(\tau^{\frac{1}{4}}, \tau^{\frac{1}{2}}\right) \mathcal{N}_{1}[F] \\
\left\|\Phi_{1}(\tau)\right\|_{L_{t}^{1} L_{x}^{2}} & \lesssim \max \left(\tau^{\frac{1}{2}-}, \tau^{\frac{1}{2}}\right) \mathcal{N}_{1}[F]
\end{aligned}
$$

for any $1 \leq q<2$ close to 2 and $\tau>0$.

Step 2: We show that $\Phi_{2}$ verifies the weak estimate,

$$
\left\|\Phi_{2}(\tau)\right\|_{L_{t}^{q} L_{x}^{2}} \lesssim \max \left(\tau^{\frac{1}{q}-\frac{1}{2}-}, \tau^{\frac{1}{2}+}\right) \mathcal{N}_{1}[F]
$$

for any $1 \leq q<2$ close to 2 .

Step 3: Using the results above we conclude that the estimate (128) holds true.

Step 4: With the help of (128) we can improve the estimate for $\Phi_{2}$ and derive the desired estimate (127).

\section{3. $L_{t}^{q} L_{x}^{2}$ estimates for $\Phi_{1}$.}

Lemma 12.4. For all $q<2$ sufficiently close to $q=2$ and any $\tau \geq 0$,

$$
\left\|\Phi_{1}(\tau)\right\|_{L_{t}^{q} L_{x}^{2}} \lesssim \max \left(\tau^{\frac{1}{4}}, \tau^{\frac{1}{2}}\right) \mathcal{N}_{1}[F]
$$

Proof : We start by estimating the expression

$$
\phi_{1}\left(\tau^{\prime}\right)=\hat{A} \cdot \nabla U\left(\tau^{\prime}\right) F+\beta \cdot U\left(\tau^{\prime}\right) F
$$

Applying Hölder inequality followed by Gagliardo-Nirenberg (39) and the estimates (132)-(134), we obtain

$$
\begin{aligned}
\left\|\phi_{1}\left(\tau^{\prime}\right)\right\|_{L_{x}^{2}} & \lesssim\left\|\hat{A} \cdot \nabla U\left(\tau^{\prime}\right) F\right\|_{L_{x}^{2}}+\left\|\beta \cdot U\left(\tau^{\prime}\right) F\right\|_{L_{x}^{2}} \\
& \lesssim\|\hat{A}\|_{L_{x}^{4}} \cdot\left\|\nabla U\left(\tau^{\prime}\right) F\right\|_{L_{x}^{4}}+\|\beta\|_{L_{x}^{2}} \cdot\left\|U\left(\tau^{\prime}\right) F\right\|_{L_{x}^{\infty}} \\
& \lesssim\|\hat{A}\|_{L_{x}^{4}} \cdot\left\|\nabla^{2} U\left(\tau^{\prime}\right) F\right\|_{L_{x}^{2}}^{\frac{1}{2}} \cdot\left\|\nabla U\left(\tau^{\prime}\right) F\right\|_{L_{x}^{2}}^{\frac{1}{2}}+\|\beta\|_{L_{x}^{2}} \cdot\left\|U\left(\tau^{\prime}\right) F\right\|_{L_{x}^{\infty}} \\
& \lesssim\|\hat{A}\|_{L_{x}^{4}}\left(\tau^{\prime-\frac{1}{4}}+\|K\|_{L_{x}^{2}}^{\frac{p}{2(p-1)}}\right)\left\|U\left(\tau^{\prime} / 2\right) F\right\|_{H_{x}^{1}} \\
& +\left\langle\tau^{\prime-\frac{1}{4}}\right\rangle\|\beta\|_{L_{x}^{2}}\left(1+\|K\|_{L_{x}^{2}}^{\frac{1}{2(p-1)}}\right)\|F\|_{L_{x}^{4}}
\end{aligned}
$$

Remark 12.5. To simplify the exposition we will take $p=\infty$ in the above estimate. Properly speaking this is not acceptable since the estimates of lemma 12.2 do not hold true for $p=\infty$. The correct proof requires the choice of an appropriate large value of $p<\infty$ dependent on the exponent $q<2$. As long as we stay away from the critical exponent $q=2$ one can easily correct the slightly idealized exposition below. We note in fact that the restriction to $q<2$ is due to the presence of the Gauss curvature terms $\|K\|_{L_{x}^{2}}$, which were generated by the Böchner identity and 
inequality for tensors. Many of the technical complications below are due to the presence of these terms.

Hence,

$$
\left\|\phi_{1}\left(\tau^{\prime}\right)\right\|_{L_{x}^{2}} \lesssim\|\hat{A}\|_{L_{x}^{4}}\left(\tau^{\prime-\frac{1}{4}}+\|K\|_{L_{x}^{2}}^{\frac{1}{2}}\right)\left\|U\left(\tau^{\prime} / 2\right) F\right\|_{H_{x}^{1}}+\left\langle\tau^{\prime-\frac{1}{4}}\right\rangle\|\beta\|_{L_{x}^{2}}\|F\|_{L_{x}^{4}}
$$

Fix an exponent $q, 1 \leq q<2$ and use the interpolated heat flow estimate, see proposition 3.17:

$$
\left\|U\left(\tau^{\prime} / 2\right) F\right\|_{H_{x}^{1}} \lesssim\left\langle\tau^{\prime-\left(\frac{3}{4}-\frac{1}{q}\right)}\right\rangle\|F\|_{H_{x}^{1}}^{\frac{2}{q}-\frac{1}{2}}\|F\|_{L_{x}^{2}}^{\frac{3}{2}-\frac{2}{q}}
$$

Therefore,

$$
\begin{aligned}
\left\|\phi_{1}\left(\tau^{\prime}\right)\right\|_{L_{x}^{2}} & \lesssim\|\hat{A}\|_{L_{x}^{4}}\left(\tau^{\prime-\frac{1}{4}}\|F\|_{H_{x}^{1}}+\left\langle\tau^{\prime-\left(\frac{3}{4}-\frac{1}{q}\right)}\right\rangle\|K\|_{L_{x}^{2}}^{\frac{1}{2}}\|F\|_{H_{x}^{1}}^{\frac{2}{q}-\frac{1}{2}}\|F\|_{L_{x}^{2}}^{\frac{3}{2}-\frac{2}{q}}\right) \\
& +\left\langle\tau^{\prime-\frac{1}{4}}\right\rangle\|\beta\|_{L_{x}^{2}}\|F\|_{L_{x}^{4}}
\end{aligned}
$$

We now take the $L_{t}^{q}$-norm, use the assumptions A1, A2, K1

$$
\|\hat{A}\|_{L_{x}^{4} L_{t}^{\infty}},\|\beta\|_{L_{t}^{2} L_{x}^{2}},\|K\|_{L_{t}^{2} L_{x}^{2}} \lesssim 1
$$

apply the Gagliardo-Nirenberg estimate (39), and the inequality $\|F\|_{L_{t}^{\frac{2 q}{2-q}} L_{x}^{4}} \lesssim$ $\mathcal{N}_{1}[F]$ to obtain

$$
\begin{aligned}
\left\|\phi_{1}\left(\tau^{\prime}\right)\right\|_{L_{t}^{q} L_{x}^{2}} & \lesssim\left(\tau^{\prime-\frac{1}{4}}\|F\|_{L_{t}^{q} H_{x}^{1}}+\left\langle\tau^{\prime-\left(\frac{3}{4}-\frac{1}{q}\right)}\right\rangle\|K\|_{L_{t}^{2} L_{x}^{2}}^{\frac{1}{2}}\|F\|_{L_{t}^{2} H_{x}^{1}}^{\frac{2}{q}-\frac{1}{2}}\|F\|_{L_{t}^{\infty} L_{x}^{2}}^{\frac{3}{2}-\frac{2}{q}}\right) \\
& +\left\langle\tau^{\prime-\frac{1}{4}}\right\rangle\|F\|_{L_{t}^{\frac{2 q}{2-q}} L_{x}^{4}} \lesssim\left\langle\tau^{\prime-\frac{1}{4}}\right\rangle \mathcal{N}_{1}[F]
\end{aligned}
$$

Returning to the estimates for $\Phi_{1}(\tau)$ we derive

$$
\begin{aligned}
\left\|\Phi_{1}(\tau)\right\|_{L_{t}^{q} L_{x}^{2}} & \lesssim\left\|\int_{0}^{\tau} U\left(\tau-\tau^{\prime}\right) \nabla \phi_{1}\left(\tau^{\prime}\right)\right\|_{L_{t}^{q} L_{x}^{2}} \\
& \lesssim \int_{0}^{\tau}\left(\tau-\tau^{\prime}\right)^{-\frac{1}{2}}\left\|\phi_{1}\left(\tau^{\prime}\right)\right\|_{L_{t}^{q} L_{x}^{2}} \\
& \lesssim \int_{0}^{\tau}\left(\tau-\tau^{\prime}\right)^{-\frac{1}{2}}\left\langle\tau^{\prime-\frac{1}{4}}\right\rangle \mathcal{N}_{1}[F] \\
& \lesssim \max \left(\tau^{\frac{1}{4}}, \tau^{\frac{1}{2}}\right) \mathcal{N}_{1}[F]
\end{aligned}
$$

\section{6. $L_{t}^{1} L_{x}^{2}$ estimate for $\Phi_{1}$.}

In this section we derive an improved estimate for $\Phi_{1}(\tau)$ in the $L_{t}^{1} L_{x}^{2}$-norm.

Lemma 12.7. For any $\tau \geq 0$,

$$
\left\|\Phi_{1}(\tau)\right\|_{L_{t}^{1} L_{x}^{2}} \lesssim \max \left(\tau^{\frac{1}{2}-}, \tau^{\frac{1}{2}}\right) \mathcal{N}_{1}[F]
$$


Proof : Fix a sufficiently large exponent $r<\infty$. Then applying Hölder inequality followed by Gagliardo-Nirenberg estimate and heat flow estimates (133), (134), we obtain

$$
\begin{aligned}
\left\|\phi_{1}\left(\tau^{\prime}\right)\right\|_{L_{x}^{2}} & \lesssim\left\|\hat{A} \cdot \nabla U\left(\tau^{\prime}\right) F\right\|_{L_{x}^{2}}+\left\|\beta \cdot U\left(\tau^{\prime}\right) F\right\|_{L_{x}^{2}} \\
& \lesssim\|\hat{A}\|_{L_{x}^{r}}\left\|\nabla U\left(\tau^{\prime}\right) F\right\|_{L_{x}^{\frac{2 r}{r-2}}}+\|\beta\|_{L_{x}^{2}}\left\|U\left(\tau^{\prime}\right) F\right\|_{L_{x}^{\infty}} \\
& \lesssim\|\hat{A}\|_{L_{x}^{r}}\left\|\nabla^{2} U\left(\tau^{\prime}\right) F\right\|_{L_{x}^{2}}^{\frac{2}{r}}\left\|\nabla U\left(\tau^{\prime}\right) F\right\|_{L_{x}^{2} \frac{2}{r}}^{1}+\|\beta\|_{L_{x}^{2}} \cdot\left\|U\left(\tau^{\prime}\right) F\right\|_{L_{x}^{\infty}} \\
& \lesssim\|\hat{A}\|_{L_{x}^{r}}\left(\tau^{\prime-\frac{1}{r}}+\|K\|_{L_{x}^{2}}^{\frac{2 p}{r(p-1)}}\right)\left\|U\left(\tau^{\prime} / 2\right) F\right\|_{H_{x}^{1}} \\
& +\left\langle\tau^{\prime-\frac{1}{r}}\right\rangle\|\beta\|_{L_{x}^{2}}\left(1+\|K\|_{L_{x}^{2}}^{\frac{2}{r(p-1)}}\right)\|F\|_{L_{x}^{r}}
\end{aligned}
$$

Once again we set $p=\infty$, see remark 12.5 ,

$$
\left\|\phi_{1}\left(\tau^{\prime}\right)\right\|_{L_{x}^{2}} \lesssim\left(\tau^{\prime-\frac{1}{r}}+\|K\|_{L_{x}^{2}}^{\frac{2}{r}}\right)\|\hat{A}\|_{L_{x}^{r}}\|F\|_{H_{x}^{1}}+\left\langle\tau^{\prime-\frac{1}{r}}\right\rangle\|\beta\|_{L_{x}^{2}}\|F\|_{L_{x}^{r}}
$$

Taking $L_{t}^{1}$-norm and using our assumptions $\mathbf{A 1}, \mathbf{A 2}, \mathbf{K} \mathbf{1}$ we derive

$$
\begin{aligned}
\left\|\phi_{1}\left(\tau^{\prime}\right)\right\|_{L_{t}^{1} L_{x}^{2}} & \lesssim \tau^{\prime-\frac{1}{r}}\|\hat{A}\|_{L_{t}^{2} L_{x}^{r}}\|F\|_{L_{t}^{2} H_{x}^{1}}+\left\langle\tau^{\prime-\frac{1}{r}}\right\rangle\|\beta\|_{L_{t}^{2} L_{x}^{2}}\|F\|_{L_{t}^{2} L_{x}^{r}} \\
& +\|\hat{A}\|_{L_{t}^{\frac{2 r}{r-2}} L_{x}^{r}} \cdot\|K\|_{L_{t}^{2} L_{x}^{2}}^{\frac{2}{r}}\|F\|_{L_{t}^{2} H_{x}^{1}} \lesssim\left\langle\tau^{\prime-\frac{1}{r}}\right\rangle \mathcal{N}_{1}[F] .
\end{aligned}
$$

for an arbitrarily large $r$.

To derive the last estimate we have used lemma 12.8 below.

Finally we recall that $\Phi_{1}(\tau)=\int_{0}^{\tau} U\left(\tau-\tau^{\prime}\right) \nabla \phi_{1}\left(\tau^{\prime}\right)$ and proceeding as before, with $r$ arbitrarily large, we finish the proof.

Lemma 12.8. For any $2 \leq p<\infty$ and $2 \leq q<\frac{2 p}{p-4}$

$$
\|F\|_{L_{t}^{q} L_{x}^{p}} \lesssim \mathcal{N}_{1}[F]
$$

Proof : The proof follows immediately by interpolating between the following:

$$
\|F\|_{L_{t}^{2} L_{x}^{r}} \lesssim \mathcal{N}_{1}[F], \quad\|F\|_{L_{t}^{\infty} L_{x}^{4}} \lesssim \mathcal{N}_{1}[F]
$$

\section{9. $L_{t}^{q} L_{x}^{2}$ estimates for $\Phi_{2}$.}

Lemma 12.10. For any $1 \leq q<2$ and all $\tau \geq 0$,

$$
\left\|\Phi_{2}(\tau)\right\|_{L_{t}^{q} L_{x}^{2}} \lesssim \max \left(\tau^{\frac{1}{q}-\frac{1}{2}-}, \tau^{\frac{1}{2}+}\right) \mathcal{N}_{1}[F]
$$

Proof : We start by estimating the function

$$
\phi_{2}\left(\tau^{\prime}\right)=\nabla A \cdot \nabla U\left(\tau^{\prime}\right) F+\beta \cdot \nabla U\left(\tau^{\prime}\right) F
$$


Fix $r>1$ sufficiently close to $r=1$ and apply Hölder inequality followed by the Gagliardo-Nirenberg estimate,

$$
\begin{aligned}
\left\|\phi_{2}\left(\tau^{\prime}\right)\right\|_{L_{x}^{r}} & \lesssim\left(\|\nabla A\|_{L_{x}^{2}}+\|\beta\|_{L_{x}^{2}}\right)\left\|\nabla U\left(\tau^{\prime}\right) F\right\|_{L_{x}^{\frac{2 r}{2-r}}} \\
& \lesssim\left(\|\nabla A\|_{L_{x}^{2}}+\|\beta\|_{L_{x}^{2}}\right)\left\|\nabla^{2} U\left(\tau^{\prime}\right) F\right\|_{L_{x}^{2} \frac{2}{r}}^{2}\left\|\nabla U\left(\tau^{\prime}\right) F\right\|_{L_{x}^{2}}^{\frac{2}{r}} .
\end{aligned}
$$

We now use estimate (134) to obtain

$$
\left\|\phi_{2}\left(\tau^{\prime}\right)\right\|_{L_{x}^{r}} \lesssim\left(\|\nabla A\|_{L_{x}^{2}}+\|\beta\|_{L_{x}^{2}}\right)\left(\tau^{-\frac{r-1}{r}}+\|K\|_{L_{x}^{2}}^{2 \frac{p(r-1)}{r(p-1)}}\right)\left\|U\left(\tau^{\prime} / 2\right) F\right\|_{H_{x}^{1}}
$$

Proceeding in the spirit of remark 12.5 we set $r=1$ on the right hand side and replace the above inequality with,

$$
\left\|\phi_{2}\left(\tau^{\prime}\right)\right\|_{L_{x}^{r}} \lesssim\left(\|\nabla A\|_{L_{x}^{2}}+\|\beta\|_{L_{x}^{2}}\right)\left\|\nabla U\left(\tau^{\prime} / 2\right) F\right\|_{L_{x}^{2}}
$$

For a given exponent $q$ we use the standard heat flow estimates $\left\|\nabla U\left(\tau^{\prime} / 2\right) F\right\|_{L_{x}^{2}} \lesssim$ $\tau^{\prime-\frac{1}{2}}\|F\|_{L_{x}^{2}}$ and $\left\|\nabla U\left(\tau^{\prime} / 2\right) F\right\|_{L_{x}^{2}} \lesssim\|\nabla F\|_{L_{x}^{2}}$ to write

$$
\left\|\nabla U\left(\tau^{\prime} / 2\right) F\right\|_{L_{x}^{2}} \lesssim \tau^{\prime-\left(1-\frac{1}{q}\right)}\|\nabla F\|_{L_{x}^{2}}^{\frac{2}{q}-1}\|F\|_{L_{x}^{2}}^{2-\frac{2}{q}}
$$

Now taking the $L_{t}^{q}$-norm and using, $\|\nabla A\|_{L_{t}^{2} L_{x}^{2}},\|\beta\|_{L_{t}^{2} L_{x}^{2}} \lesssim \Delta_{0} \lesssim 1, \quad$ we derive with the help of Gagliardo-Nirenberg estimate that

$$
\begin{aligned}
\left\|\phi_{2}\left(\tau^{\prime}\right)\right\|_{L_{t}^{q} L_{x}^{r}} & \lesssim \tau^{\prime-\left(1-\frac{1}{q}\right)}\|\nabla F\|_{L_{t}^{2} L_{x}^{2}}^{\frac{2}{q}-1}\|F\|_{L_{t}^{\infty} L_{x}^{2}}^{2-\frac{2}{q}}+\|\nabla F\|_{L_{t}^{q} L_{x}^{2}} \\
& \lesssim\left\langle\tau^{\prime-\left(1-\frac{1}{q}\right)}\right\rangle \mathcal{N}_{1}[F]
\end{aligned}
$$

Returning to the estimates for $\Phi_{2}(\tau)$ and applying the dual weak Bernstein inequality $L^{2} \rightarrow L^{r}$, see (49), for some $r>1$ sufficiently close to $r=1$, we obtain

$$
\begin{aligned}
\left\|\Phi_{2}\left(\tau^{\prime}\right)\right\|_{L_{t}^{q} L_{x}^{2}} & \lesssim\left\|\int_{0}^{\tau} U\left(\tau-\tau^{\prime}\right) \phi_{2}\left(\tau^{\prime}\right)\right\|_{L_{t}^{q} L_{x}^{2}} \\
& \lesssim \int_{0}^{\tau}\left(\tau-\tau^{\prime}\right)^{-\left(\frac{1}{r}-\frac{1}{2}\right)}\left\|\phi_{2}\left(\tau^{\prime}\right)\right\|_{L_{t}^{q} L_{x}^{r}} \\
& \lesssim \int_{0}^{\tau}\left(\tau-\tau^{\prime}\right)^{-\left(\frac{1}{r}-\frac{1}{2}\right)}\left\langle\tau^{\prime}\right\rangle^{-\left(1-\frac{1}{q}\right)} \mathcal{N}_{1}[F] \\
& \lesssim \max \left(\tau^{\frac{1}{2}+\frac{1}{q}-\frac{1}{r}}, \tau^{\frac{3}{2}-\frac{1}{r}}\right) \mathcal{N}_{1}[F] \\
& \lesssim \max \left(\tau^{\frac{1}{q}-\frac{1}{2}}, \tau^{\frac{1}{2}+}\right) \mathcal{N}_{1}[F]
\end{aligned}
$$

We can now finish the proof of proposition 12.1 for the case of $q=1$. Recall that

$$
\left[P_{k}, \nabla_{L}\right] F=\int_{0}^{\infty} m_{k}(\tau) \Phi(\tau) d \tau
$$


where $\Phi(\tau)=\Phi_{1}(\tau)+\Phi_{2}(\tau)$. Combining the results of lemmas 12.7 and 12.10 we derive

$$
\begin{aligned}
\left\|\left[P_{k}, \nabla_{L}\right] F\right\|_{L_{t}^{1} L_{x}^{2}} & \lesssim \mathcal{N}_{1}[F] \cdot \int_{0}^{\infty} m_{k}(\tau) \max \left(\tau^{\frac{1}{2}-}, \tau^{\frac{1}{2}+}\right) \\
& \lesssim \mathcal{N}_{1}[F] \cdot\left(\int_{0}^{1} m_{k}(\tau) \tau^{\frac{1}{2}-}+\int_{1}^{\infty} m_{k}(\tau) \cdot \tau^{\frac{1}{2}+}\right) \\
& \lesssim \mathcal{N}_{1}[F] \cdot\left(2^{-k+} \int_{0}^{2^{2 k}} m(\tau) \tau^{\frac{1}{2}-}+2^{-k-} \int_{2^{2 k}}^{\infty} m(\tau) \tau^{\frac{1}{2}+}\right) \\
& \lesssim 2^{-k+} \cdot \mathcal{N}_{1}[F]
\end{aligned}
$$

To obtain the estimate for the term $\left\|\nabla\left[P_{k}, \nabla_{L}\right] F\right\|_{L_{t}^{1} L_{x}^{2}}$ we need to control $\nabla \Phi_{1}(\tau)$ and $\nabla \Phi_{2}(\tau)$. Observe that the tensor-fields $\Phi_{j}(\tau), j=1,2$ are the solutions of the respective heat equations

$$
\begin{aligned}
& \partial_{\tau} \Phi_{1}-\Delta \Phi_{1}=\nabla \phi_{1},\left.\quad \Phi_{1}\right|_{\tau=0}=0, \\
& \partial_{\tau} \Phi_{2}-\Delta \Phi_{2}=\phi_{2},\left.\quad \Phi_{2}\right|_{\tau=0}=0
\end{aligned}
$$

The standard heat flow estimates imply that

$$
\begin{aligned}
&\left\|\nabla \Phi_{1}\right\|_{L_{\tau}^{2} L_{x}^{2}} \lesssim\left\|\phi_{1}\right\|_{L_{\tau}^{2} L_{x}^{2}}, \\
&\left\|\nabla \Phi_{2}\right\|_{L_{\tau}^{2} L_{x}^{2}} \lesssim\left\|\phi_{2}\right\|_{L_{\tau}^{\frac{2 p}{3 p-2}} L_{x}^{p}}, \quad 1 \leq p \leq 2
\end{aligned}
$$

where \|\|$_{L_{\tau}^{2}}$ refer to the $L^{2}$ norm on the interval $[0, \tau]$. The proof of 141 follows by multiplying the heat equation for $\Phi_{1}$ by $\Phi_{1}$ and integrating by parts. Estimate (142) follows in the same manner, replacing the integration by parts by an appropriate use of Hölder and interpolation.

Taking the $L_{\tau}^{2}$ norm of the equations (136) and (138) followed by the $L_{t}^{1}$ norm we derive, for any $p>1$ sufficiently close to 1 and all sufficiently large $r$,

$$
\begin{aligned}
\left\|\phi_{1}\right\|_{L_{t}^{1} L_{\tau}^{2} L_{x}^{2}} & \lesssim \max \left(\tau^{\frac{1}{2}}, \tau^{\frac{1}{2}-\frac{1}{r}}\right) \cdot \mathcal{N}_{1}[F] \\
\left\|\phi_{2}\right\|_{L_{t}^{1} L_{\tau}^{\frac{2 p}{3 p-2}} L_{x}^{p}} & \lesssim \tau^{\frac{3 p-2}{2 p}} \cdot \mathcal{N}_{1}[F]
\end{aligned}
$$

Therefore,

$$
\begin{aligned}
\left\|\nabla \Phi_{1}\right\|_{L_{t}^{1} L_{\tau}^{2} L_{x}^{2}} & \lesssim \max \left(\tau^{\frac{1}{2}}, \tau^{\frac{1}{2}-}\right) \cdot \mathcal{N}_{1}[F] \\
\left\|\nabla \Phi_{2}\right\|_{L_{t}^{1} L_{\tau}^{2} L_{x}^{2}} & \lesssim \max \left(\tau^{\frac{1}{2}}, \tau^{\frac{1}{2}-}\right) \cdot \mathcal{N}_{1}[F]
\end{aligned}
$$

One can, by a standard argument, convert the above into the following weighted estimates,

$$
\begin{aligned}
&\left\|\min \left(\tau^{-\frac{1}{2}-}, \tau^{-\frac{1}{2}+}\right) \nabla \Phi_{1}\right\|_{L_{t}^{1} L_{\tau}^{2} L_{x}^{2}} \lesssim \mathcal{N}_{1}[F] \\
&\left\|\min \left(\tau^{-\frac{1}{2}-}, \tau^{-\frac{1}{2}+}\right) \nabla \Phi_{2}\right\|_{L_{t}^{1} L_{\tau}^{2} L_{x}^{2}} \lesssim \mathcal{N}_{1}[F]
\end{aligned}
$$

Thus

$$
\begin{aligned}
\left\|\nabla\left[P_{k}, \nabla_{L}\right] F\right\|_{L_{t}^{1} L_{x}^{2}} & \lesssim\left\|\int_{0}^{\infty} m_{k}(\tau) \nabla \Phi(\tau)\right\|_{L_{t}^{1} L_{x}^{2}} \\
& \lesssim\left\|\max \left(\tau^{\frac{1}{2}-}, \tau^{\frac{1}{2}+}\right) m_{k}(\tau)\right\|_{L_{\tau}^{2}}\left\|\min \left(\tau^{-\frac{1}{2}+}, \tau^{-\frac{1}{2}-}\right) \nabla \Phi_{j}\right\|_{L_{t}^{1} L_{\tau}^{2} L_{x}^{2}} \\
& \lesssim 2^{k} 2^{-k+} \mathcal{N}_{1}[F]
\end{aligned}
$$


To finish the proof of proposition 12.1 for an arbitrary $q<2$ we first need to obtain improved estimates for $\Phi_{2}$.

\subsection{Improved estimates for $\Phi_{2}$.}

Lemma 12.12. For all $1 \leq q<2$ and any $\tau \geq 0$

$$
\left\|\Phi_{2}(\tau)\right\|_{L_{t}^{q} L_{x}^{2}} \lesssim \max \left(\tau^{\frac{1}{4}-}, \tau^{\frac{1}{2}}\right) N_{1}[F]
$$

Proof : We start by proceeding in the same way as in the estimates for $\Phi_{2}(\tau)$ of lemma 12.10 until equation (138),

$$
\left\|\phi_{2}\left(\tau^{\prime}\right)\right\|_{L_{x}^{r}} \lesssim\left(\|\nabla A\|_{L_{x}^{2}}+\|\beta\|_{L_{x}^{2}}\right)\left\|\nabla U\left(\tau^{\prime} / 2\right) F\right\|_{L_{x}^{2}}
$$

Taking the $L_{t}^{q}$-norm,

$$
\left\|\phi_{2}\left(\tau^{\prime}\right)\right\|_{L_{t}^{q} L_{x}^{r}} \lesssim\left\|\nabla U\left(\tau^{\prime} / 2\right) F\right\|_{L_{t}^{\frac{2 q}{2-q}} L_{x}^{2}} \lesssim\left\|\nabla U\left(\tau^{\prime} / 2\right) F\right\|_{L_{t}^{\infty} L_{x}^{2}}
$$

We now estimate the term $\left\|\nabla U\left(\tau^{\prime} / 2\right) F\right\|_{L_{t}^{\infty} L_{x}^{2}}$. Squaring, and integrating by parts in $t$ and commuting $\nabla_{L}$ with $\nabla$ and then with $U\left(\tau^{\prime} / 2\right)$ and then using the heat flow estimates we derive,

$$
\begin{aligned}
\left\|\nabla U\left(\tau^{\prime} / 2\right) F\right\|_{L_{t}^{\infty} L_{x}^{2}}^{2} & \lesssim \int_{\mathcal{H}} \nabla_{L} \nabla U\left(\tau^{\prime} / 2\right) F \cdot \nabla U\left(\tau^{\prime} / 2\right) F+\left(\mathcal{N}_{1}[F]\right)^{2} \\
& \lesssim \int_{\mathcal{H}} \nabla \nabla_{L} U\left(\tau^{\prime} / 2\right) F \cdot \nabla U\left(\tau^{\prime} / 2\right) F \\
& +\left\|\left[\nabla, \nabla_{L}\right] U\left(\tau^{\prime} / 2\right) F\right\|_{L_{t}^{1} L_{x}^{2}}\left\|\nabla U\left(\tau^{\prime} / 2\right) F\right\|_{L_{t}^{\infty} L_{x}^{2}}+\left(\mathcal{N}_{1}[F]\right)^{2} \\
& \lesssim \int_{\mathcal{H}} \nabla U\left(\tau^{\prime} / 2\right) \nabla_{L} F \cdot \nabla U\left(\tau^{\prime} / 2\right) F \\
& +\left\|\left[\nabla_{L}, U\left(\tau^{\prime} / 2\right)\right] F\right\|_{L_{t}^{1} L_{x}^{2}}\left\|\Delta U\left(\tau^{\prime} / 2\right) F\right\|_{L_{t}^{\infty} L_{x}^{2}} \\
& +\left\|\left[\nabla, \nabla_{L}\right] U\left(\tau^{\prime} / 2\right) F\right\|_{L_{t}^{1} L_{x}^{2}}\left\|\nabla U\left(\tau^{\prime} / 2\right) F\right\|_{L_{t}^{\infty} L_{x}^{2}}+\left(\mathcal{N}_{1}[F]\right)^{2} \\
& \lesssim\left\langle\tau^{\prime-\frac{1}{2}}\right\rangle\left(\mathcal{N}_{1}[F]\right)^{2}+\left\|\left[\nabla_{L}, U\left(\tau^{\prime} / 2\right)\right] F\right\|_{L_{t}^{1} L_{x}^{2}}\left\|\Delta U\left(\tau^{\prime} / 2\right) F\right\|_{L_{t}^{\infty} L_{x}^{2}} \\
& +\left\|\left[\nabla, \nabla_{L}\right] U\left(\tau^{\prime} / 2\right) F\right\|_{L_{t}^{1} L_{x}^{2}}\left\|\nabla U\left(\tau^{\prime} / 2\right) F\right\|_{L_{t}^{\infty} L_{x}^{2}}
\end{aligned}
$$

Thus, since

$$
\begin{aligned}
&\left\|\Delta U\left(\tau^{\prime} / 2\right) F\right\|_{L_{t}^{\infty} L_{x}^{2}} \lesssim\left(\tau^{\prime}\right)^{-1}\|F\|_{L_{t}^{\infty} L_{x}^{2}} \lesssim\left(\tau^{\prime}\right)^{-1} \mathcal{N}_{1}[F] \\
&\left\|\nabla U\left(\tau^{\prime} / 2\right) F\right\|_{L_{t}^{\infty} L_{x}^{2}} \lesssim\left\langle{\tau^{\prime}}^{-\frac{1}{4}}\right\rangle \mathcal{N}_{1}[F]+\tau^{\prime-\frac{1}{2}}\left\|\left[\nabla_{L}, U\left(\tau^{\prime} / 2\right)\right] F\right\|_{L_{t}^{1} L_{x}^{2}}^{\frac{1}{2}} \cdot\left(\mathcal{N}_{1}[F]\right)^{\frac{1}{2}} \\
&+\left\|\left[\nabla, \nabla_{L}\right] U\left(\tau^{\prime} / 2\right) F\right\|_{L_{t}^{1} L_{x}^{2}}
\end{aligned}
$$

The results of lemmas 12.7 and 12.12 imply that

$$
\left\|\left[\nabla_{L}, U\left(\tau^{\prime} / 2\right)\right] F\right\|_{L_{t}^{1} L_{x}^{2}} \lesssim \max \left(\tau^{\prime \frac{1}{2}-}, \tau^{\prime \frac{1}{2}+}\right) \mathcal{N}_{1}[F]
$$

On the other hand, observe that we can write the commutator formula for $\left[\nabla, \nabla_{L}\right]$ in the form $\left[\nabla, \nabla_{L}\right]=\hat{A} \cdot \nabla+\beta \cdot$. Therefore remembering the definition of $\phi_{1}(\tau)$,

$$
\left[\nabla, \nabla_{L}\right] U\left(\tau^{\prime} / 2\right) F \approx \phi_{1}\left(\tau^{\prime} / 2\right)
$$


Thus, in view of 137 ,

$$
\left\|\left[\nabla, \nabla_{L}\right] U\left(\tau^{\prime} / 2\right) F\right\|_{L_{t}^{1} L_{x}^{2}} \lesssim\left\langle\left(\tau^{\prime}\right)^{0-}\right\rangle \cdot \mathcal{N}_{1}[F]
$$

Returning to (143) we infer that

$$
\left\|\nabla U\left(\tau^{\prime} / 2\right) F\right\|_{L_{t}^{\infty} L_{x}^{2}} \lesssim\left\langle{\tau^{\prime}}^{-\frac{1}{4}-}\right\rangle \mathcal{N}_{1}[F] .
$$

It then follows that

$$
\left\|\phi_{2}\left(\tau^{\prime}\right)\right\|_{L_{t}^{q} L_{x}^{r}} \lesssim\left\|\nabla U\left(\tau^{\prime} / 2\right) F\right\|_{L_{t}^{\infty} L_{x}^{2}} \lesssim\left\langle{\tau^{\prime}}^{-\frac{1}{4}-}\right\rangle \cdot \mathcal{N}_{1}[F] .
$$

Proceeding as in lemma 12.10 we obtain for $r>1$ sufficiently close to $r=1$,

$$
\begin{aligned}
\left\|\Phi_{2}(\tau)\right\|_{L_{t}^{q} L_{x}^{2}} & \lesssim\left\|\int_{0}^{\tau} U\left(\tau-\tau^{\prime}\right) \phi_{2}\left(\tau^{\prime}\right)\right\|_{L_{t}^{q} L_{x}^{2}} \\
& \lesssim \int_{0}^{\tau}\left(\tau-\tau^{\prime}\right)^{-\left(\frac{1}{r}-\frac{1}{2}\right)}\left\|\phi_{2}\left(\tau^{\prime}\right)\right\|_{L_{t}^{q} L_{x}^{r}} \\
& \lesssim \int_{0}^{\tau}\left(\tau-\tau^{\prime}\right)^{-\left(\frac{1}{r}-\frac{1}{2}\right)}\left\langle\tau^{\prime-\frac{1}{4}-}\right\rangle \mathcal{N}_{1}[F] \\
& \lesssim \max \left(\tau^{\frac{5}{4}-\frac{1}{r}-}, \tau^{\frac{3}{2}-\frac{1}{r}}\right) N_{1}[F] \\
& \lesssim \max \left(\tau^{\frac{1}{4}-}, \tau^{\frac{1}{2}}\right) N_{1}[F]
\end{aligned}
$$

We can now finish the proof of proposition 12.1.

Combining the results of lemmas 12.4 and 12.12 we derive

$$
\begin{aligned}
\left\|\left[P_{k}, \nabla_{L}\right] F\right\|_{L_{t}^{q} L_{x}^{2}} & \lesssim \int_{0}^{\infty} m_{k}(\tau) \max \left(\tau^{\frac{1}{4}-}, \tau^{\frac{1}{2}}\right) \mathcal{N}_{1}[F] \\
& \lesssim \int_{0}^{1} m_{k}(\tau) \tau^{\frac{1}{4}-} \mathcal{N}_{1}[F]+\int_{1}^{\infty} m_{k}(\tau) \tau^{\frac{1}{2}+} \mathcal{N}_{1}[F] \\
& \lesssim 2^{-\frac{k}{2}+} \int_{0}^{2^{2 k}} m(\tau) \tau^{\frac{1}{4}-} \mathcal{N}_{1}[F]+2^{-k} \int_{2^{2 k}}^{\infty} m(\tau) \tau^{\frac{1}{2}} \mathcal{N}_{1}[F] \\
& \lesssim 2^{-\frac{k}{2}+} \mathcal{N}_{1}[F]
\end{aligned}
$$

Arguing as in the case of the $L_{t}^{1} L_{x}^{2}$ estimates can we also obtain that

$$
\left\|\nabla\left[P_{k}, \nabla_{L}\right] F\right\|_{L_{t}^{q} L_{x}^{2}} \lesssim 2^{k} 2^{-\frac{k}{2}+} \mathcal{N}_{1}[F]
$$

\section{REFERENCES}

[B] J.M. Bony Calcul Symbolique et propagation des singularité pour les equations aux dérivées partielles nonlinéares, Ann. Sci. École Norm. Sup 14, 209-256.

[Chr-Kl] D.Christodoulou and S. Klainerman. The Global Nonlinear Stability of the Minkowski Space. Princeton Mathematical Series, 41. Princeton University Press, 1993

[K11] S. Klainerman, PDE as a unified subject Special Volume GAFA 2000, 279-315

[Kl-Nic] S. Klainerman and F. Nicolo, The Evolution problem in General Relativity, Progress in Mathematical Physics 25, Birkhauser. 
[Kl-Rodn1] S. Klainerman and I. Rodnianski, Causal geometry of Einstein-Vacuum spacetimes with finite curvature flux. preprint

[Kl-Rodn2] S. Klainerman and I. Rodnianski, A geometric version of Litlewood-Paley theory. preprint

[Stein1] E. Stein Harmonic analysis Princeton University Press, 1993

[Stein2] E. Stein Topics in harmonic analysis related to Littlewood-Paley theory, Annals of Mathematical Studies, Princeton University Press 63, 1970

Department of Mathematics, Princeton University, Princeton NJ 08544

E-mail address: $\quad$ seri@math.princeton.edu

Department of Mathematics, Princeton University, Princeton NJ 08544

E-mail address: irod@math.princeton.edu 